\title{
The molecular mechanisms underlying skeletal and cardiac muscle remodeling in the hibernating thirteen-lined ground squirrel
}

\author{
By \\ Yichi (Tony) Zhang
}

B.Sc. Kinesiology, Queen's University, 2014

A Thesis Submitted to the Faculty of Graduate Studies and Research in partial fulfillment of the requirements for the degree of

\author{
Master of Science \\ Department of Biology \\ Carleton University \\ Ottawa, Ontario, Canada
}

(C) 2016

Yichi Zhang 
The undersigned hereby recommend to the Faculty of Graduate Studies and Research acceptance of his thesis

\title{
The molecular mechanisms underlying skeletal and cardiac muscle remodeling in the hibernating thirteen-lined ground squirrel
}

\author{
Submitted by \\ Yichi (Tony) Zhang \\ In partial fulfillment of the requirements for the degree of Master of Science
}

Chair, Department of Biology

Thesis Supervisor

Carleton University 


\begin{abstract}
$\underline{\text { Abstract }}$
The thirteen-lined ground squirrel (Ictidomys tridecemlineatus) survives winters by hibernating, whereby body temperature $\left(\mathrm{T}_{\mathrm{b}}\right)$ cycles between $4^{\circ} \mathrm{C}$ during torpor and $37^{\circ} \mathrm{C}$ during arousal. Each organ/tissue of the hibernator must make specific adjustments that allow the ground squirrel to maintain or readjust physiological function during hibernation. The remodeling that occurs in skeletal and cardiac muscle is unique to hibernators, and it is fascinating as a natural means of avoiding physiological dysfunction in these tissues. The purpose of this thesis is to evaluate the molecular mechanisms underlying muscle remodeling in both tissues. It was identified that calcium signaling activates the NFAT-calcineurin pathway, leading to increased expression of hypertrophy-promoting targets in both skeletal and cardiac muscle during torpor. In addition, we found that there is differential expression and activity of transcription factors (Foxo, MyoG) and ubiquitin ligases (MAFbx and MURF1) that promote muscle atrophy in the two tissues being studied.
\end{abstract}




\section{Acknowledgements}

First off, I would like to thank Dr. Ken Storey for graciously offering to take me on as a Master's student and for his constant support and mentorship. This next paragraph is dedicated to thanking the man, the myth, the legend. Throughout the summer of 2014, I contemplated where I wanted to start my Master's, and to be honest I was initially very unsure of my decision to come to Carleton. I knew that I love basic science, but I wanted to apply my findings to medicine, and so I thought long and hard about starting a Master's at the UOttawa Faculty of Medicine. However, now that I look back on it, I couldn't be happier with my decision to join the Storey Lab. Initially it didn't start off so great, I was preoccupied with rowing, volunteering, and shadowing doctors while getting "my" work done at the lab. I know that you wanted me to be more of a leader, but that wasn't what I wanted from my Master's - I wanted to fly under the radar, get my results and publications, then get into medical school (I'm always going to be honest with you, no BS). Despite this disagreement, you let me do what I do best, which is focusing on my own bench work, writing it up and publishing it, then plan the subsequent projects and repeating this process over and over again. I had a very systematic approach to my Master's, and you let me focus on my approach without much deviation (i.e. spending a lot of time teaching undergrads). This is what makes you a great lab manager and teacher; you know the individual strengths of your team members, and you let them play their role. While I decide what to do next, I just want to let you know that my accomplishments and success over the past two years would not have been possible had I not decided joined the Storey Lab under your tutelage. Therefore, should I be blessed with an opportunity to take the next step in my pilgrimage (to become an orthopedic surgeon and NBA team doctor one day), I'd say that half of the credit for me getting this far has to go to you. I will continue to look up to you as a mentor, teacher, and role-model.

I will remember how you take every opportunity to chirp Lebron and my affection for him. To be honest, I love LeBron the person more so than the basketball player, but I want and need to see him succeed in bringing a title back to Cleveland because I want to know that a kind, philanthropic, deeply friend-oriented, family man and good guy in general can make it to the top of the mountain and be recognized as one of the greatest if not the greatest alongside all the coldblooded killers in the history books - Jordan, Curry, Bird, Kareem, as well as the ringless Iverson and Westbrook - who are recognized for their greatness. Lastly, you've asked me a few times to consider staying in the lab for a $\mathrm{PhD}$, and the reason I can't do it, as you probably already know, is because I have not only big and ambitious plans outside of research, but within research as well. I believe that the only way to get better and improve in your craft is to test yourself, to challenge and learn from those who are better than you at what you do. Therefore, should I continue down the research path and start a PhD, I need to test myself against the top medical researchers in the business so that I know if I'm cut out for doing this for the rest of my life. You are like Dirk Nowitzki, who is a legend, a revolutionary player, and someone who has stayed true to himself and became the best at what he does (just like you have focused on the same field of research and became the best in your field). But as you know, I identify more with LeBron, and so I need to test myself against all the greats in pursuit of becoming the G.O.A.T (Greatest of All Time). Call me conceded, but although I know this journey is going to be stressful and difficult, my goals have kept me and will keep me going.

Ok, so after that long epiphany, I must also thank the others who have contributed to my accomplishments and the fun (sometimes too much fun) times I have had over the past two 
years. Thanks first of all to Jan Storey, mother of dragons, although most of us in the lab are just simple hatchlings (I think there's even a few dragons who are still in their shell...). Your edits taught me what an excellent manuscript and poster should look like; you made me a better writer and researcher. Also, you are a human encyclopedia and Ken knows (even if he doesn't show it sometimes) that you are the engine that keeps the lab running. However, Sanoji W is starting to exert her dominance and take control of your dragons as their step-mother! Beware!

To my dear friends in the lab, all of you have helped me improve as a researcher and as a person in one way or another. Some have provided me with constant support (even when I didn't need it), shout out to Sanoji W, Kama S, and Rasha A (the Ugly Sisters from Cinderella though you're anything but ugly). Some have changed my views and values for the better through our DMCs (Deep Meaningful Conversations), holler at Mike S (aka BFF), Bryan L (aka Luu), and Alex W (aka Abdul). Some have changed my views and values for the worse; don't worry this isn't a bad thing Sam W; I'd rather see myself live long enough to become a villain than die a hero. Also shout out to Sam L and Hanane HM for checking my privilege and commanding my respect with your hard work and dedication \#thefuture. There are plenty of people I haven't gotten the chance to thank, but that doesn't mean I don't appreciate your support. I'm the type of person who is introverted and likes to keep things to himself. You might not see me as such, but trust me on this one, I wish I got to know everyone in the lab equally but I can't help but be the way I am. Sanoji W knows this better than anyone, and despite your flaws (even though you think you're flawless), I know that your meddling in my business is your way of showing that you care. For that, I will always cherish your friendship. Lastly, thank you to the undergrads in the Storey Lab as well, you know who you are! Storey Lab will always be a kid's zone, never change!

I would like to thank my rowing team for motivating me, teaching me, and supporting me. You're a fun group of cats, especially when we're not waking up at 4 am to get to practice and row at freezing temperatures. Thanks to those on the team who played intramural basketball in our offseason and especially to Mikayla Arends for putting together the team, it was a blast (even if we only won one game). Thanks to Coach Ed for putting up with all the times we broke our boats and oars, and with all my missed practices when I had pneumonia. Thanks to Coach Matt Noël for teaching this novice to row. Thanks to Coach Martin Rowland for teaching me how to erg properly...somewhat (lol, bad habits are hard to correct). Special shout out to Michael Mikolainis, Jeffrey Parkhouse, and Jason Sukstorf for making the transition from Novice to Varsity with me. Also, shout out to Vince O'Shaughnessey for being my pair (that never actually raced) partner. Lastly, Darren Major, you da best. Also, fyi Ken, we do not just row around pointlessly in circles every morning, if anything we row in rectangles. Get your geometry right!

Lastly, I would like to thank my parents, without whom I would not have been blessed with life, and none of this would have been possible. Thanks to my dad for being the glue that holds this family together, and to my mom for always pushing me to work harder and to reach for the stars. Thanks also to all my friends, especially those who rose up with me during our undergrad at Queen's, and my friends at UOttawa and Carleton as well. You are truly the ones who kept me sane when I felt like I was going insane, you who gave me hope when I felt hopeless. Regardless of whether or not we will be colleagues in the future, I will take every opportunity to repay you for all the kindness and support you have shown me.

"Be so good they can't ignore you"

- Damian Lillard aka Dame Dolla 


\section{Table of Contents}

Title Page

Abstract

iii

Acknowledgements

iv

Table of Contents

vi

List of Abbreviations

vii

List of Figures

xii

\section{List of Appendices}

Chapter 1

General Introduction

1

Chapter 2

General Materials and Methods

16

Chapter 3

Expression of nuclear factor of activated $\mathrm{T}$ cells

26

and downstream muscle-specific proteins in ground squirrel skeletal muscle during hibernation

Chapter 4

Nuclear factor of activated $\mathrm{T}$ cells regulates cardiac

52

hypertrophy through calcium signaling during hibernation

Chapter 5

Chapter 6

Chapter 7

Publication List

References

Appendices
Regulation of Foxo4 and MyoG promotes skeletal

64 muscle atrophy during torpor in ground squirrels

Transcriptional activation of muscle atrophy

82 promotes cardiac muscle remodeling during mammalian hibernation in ground squirrels

General Discussion

100

117

121

145 


\section{List of Abbreviations}

AIH - autoinhibitory domain

AMPK - AMP-activated protein kinase

APS - ammonium persulfate

ATP - adenosine triphosphate

bp - base pairs

CAM - calmodulin

CAMKIV - calmodulin-dependent protein kinase IV

CBP - CREB-binding protein

CDK - cyclin-dependent kinase

$\mathrm{ddH}_{2} \mathrm{O}$ - double distilled water

DPI-ELISA - DNA-protein interaction enzyme-linked immunosorbent assay

DTT - dithiolthreitol

Dyrk1a - dual-specificity tyrosine-(Y)-phosphorylation regulated kinase

EA - early arousal

EC - euthermic control

EDTA - ethylenediamine tetraacetic acid 
EMBL-EBI - European Molecular Biology Laboratory - European Bioinformatics Institute

$\mathrm{EN}$ - entrance into torpor

ER - euthermic room temperature

ET - early torpor

Foxo - forkhead box transcription factors of the $\mathrm{O}$ subclass

GSK $3 \beta$ - glycogen synthase kinase 3 beta

GTP - guanosine triphosphate

$\mathrm{H}_{2} \mathrm{O}_{2}$ - hydrogen peroxide

HDAC - histone deacetylase

Hepes - 4-(2-hydroxyethyl)-1-piperazineethanesulfonic acid

HRP - horse radish peroxidase

HSF1 - heat shock transcription factor 1

JNK - Jun N-terminal kinase

IA - interbout arousal

iTRAQ - isobaric tag for relative and absolute quantification technology

$\mathrm{kDa}$ - kilodalton

LA - late arousal 
$\mathrm{LT}$ - late torpor

MAFbx - muscle atrophy F-Box

MEF2 - myocyte enhancer factor-2

MHC - myosin heavy chain

MK - MAPK-activated protein kinase

MLC - myosin light chain

MURF1 - muscle Ring Finger 1

MyoD - muscle differentiation protein

MyoG - myogenin

NIH - National Institute of Health

NINDS - National Institute of Neurological Disorders and Stroke

NCBI - National Center for Biotechnology Information

NFAT - nuclear factor of activated T cells

PAGE - polyacrylamide gel electrophoresis

PBS - phosphate buffer saline

PBST - phosphate buffer saline with Tween

PGC-1 $\alpha-$ PPAR $\gamma$ coactivator 1 -alpha

PI3K - Phosphoinositide 3-kinase 
PKA - protein kinase A

$\mathrm{PKB} / \mathrm{Akt}$ - protein kinase B

PMSF - phenylmethanesulfonylfluoride

$\operatorname{PPAR} \gamma$ - peroxisome proliferator-activated receptor gamma

PVDF - polyvinylidine fluoride

Ralbp1 - Ral binding protein 1

$\mathrm{RT}$ - room temperature

SDS - sodium dodecyl sulfate/lauryl sulphate

Ser - serine

$\mathrm{T}_{\mathrm{b}}$ - body temperature

TBS - tris buffer saline

TBST - tris buffer saline with Tween

TEMED - N, N, N', N'-tetramethylethylenediamine

$\mathrm{TF}$ - transcription factors

$\mathrm{Thr}$ - threonine

TMB - tetramethylbenzidine

TNF $\alpha$ - tumor Necrosis Factor $\alpha$ 
Tris - tris (hydroxymethyl) aminomethane

USDA - United State Department of Agriculture

UPS - ubiquitin proteasome system 


\section{List of Figures}

Figure 1.1 Ground squirrel avoidance of skeletal muscle wasting.

Figure 1.2 Ground squirrel cardiac dimensions during active season and hibernation.

Figure 1.3 Schematic representation of the NFAT-calcineurin pathway and its regulation.

Figure 1.4 Signaling pathways involved in the regulation of muscle atrophy through the ubiquitin proteasome system (UPS).

Figure 1.5 Model for Ras-Ral pathway-dependent Foxo4 regulation of skeletal muscle atrophy.

Figure 2.1 Schematic depiction of the hibernation torpor-arousal cycle.

Figure 3.1 DNA sequence analysis of the myomaker promoter in multiple animals to find putative NFAT binding sites in ground squirrel.

Figure $3.2 \quad$ Changes in NFAT protein levels in ground squirrel skeletal muscle over the torpor-arousal cycle.

Figure 3.3 Changes in myoferlin and myomaker protein levels in ground squirrel skeletal muscle over the torpor-arousal cycle.

Figure 3.4

Changes in calcineurin, CAM, and calpain 1 protein levels in ground squirrel skeletal muscle over the torpor-arousal cycle.

Figure 3.5 Changes in NFAT-binding to DNA in ground squirrel skeletal muscle over the torpor-arousal cycle.

Figure 3.6 Effect of adjusting temperature on NFAT-DNA binding in ground squirrel skeletal muscle over the torpor-arousal cycle.

Figure 3.7 Effect of adding urea and $\mathrm{Ca}^{2+}$ on NFAT-DNA binding in ground squirrel skeletal muscle over the torpor-arousal cycle.

Figure 4.1 Changes in the protein levels of NFATs in ground squirrel cardiac muscle over the torpor-arousal cycle.

Figure 4.2 Changes in myoferlin and myomaker protein levels in ground squirrel cardiac muscle over the torpor-arousal cycle. 
Figure 4.3 Changes in calcineurin, CAM, and calpain protein levels in ground squirrel cardiac muscle over the torpor-arousal cycle.

Figure 5.1 Changes in the protein levels of Foxo4 and p-Foxo4 in ground squirrel skeletal muscle over the torpor-arousal cycle.

Figure 5.2 Changes in phosphorylation ratios of Foxo4 in ground squirrel 79 skeletal muscle over the torpor-arousal cycle.

Figure 5.3 Changes in the protein levels of MyoG, MAFbx, and MURF1 in ground squirrel skeletal muscle over the torpor-arousal cycle.

Figure 5.4

Changes in the protein levels of Ras, Ral, and Ralbp1 in ground squirrel skeletal muscle over the torpor-arousal cycle.

Figure 6.1 Changes in the protein levels of Foxo1 and p-Foxol in ground squirrel cardiac muscle over the torpor-arousal cycle.

Figure 6.2

Changes in the protein levels of Foxo3a and p-Foxo3a in 96 ground squirrel cardiac muscle over the torpor-arousal cycle.

Figure 6.3 Changes in phosphorylation ratios of Foxol and 3a in ground squirrel cardiac muscle over the torpor-arousal cycle.

Figure 6.4 Changes in the protein levels of Foxo4 and MyoG in ground squirrel cardiac muscle over the torpor-arousal cycle.

Figure 6.5

Changes in the protein levels of MAFbx and MURF1 in ground squirrel cardiac muscle over the torpor-arousal cycle.

Figure S.1 Summary figure of the relationship between the targets analyzed in the current thesis.

Table S.1 Table summarizing the changes that took place with western 148 blotting results of the NFAT-calcineurin pathway and their downstream muscle-specific proteins in skeletal muscle.

Table S.2 Table summarizing the changes that took place with the DPI149 ELISA results of NFATc1, c3, and c4 in skeletal muscle.

Table S.3 Table summarizing the changes that took place with the 150 western blotting results of the NFAT-calcineurin pathway and their downstream muscle-specific proteins in cardiac muscle. 
Table S.4 Table summarizing the changes that took place with the western blotting results of the Ras-Ral pathway, Foxo4 and its phosphorylated forms, as well as Myogenin their downstream E3 ubiquitin ligase proteins in skeletal muscle.

Table S.5

Table summarizing the changes that took place with the western blotting results of Foxo1, Foxo3a, Foxo4 and its phosphorylated forms, as well as Myogenin their downstream E3 ubiquitin ligase proteins in cardiac muscle. 
Chapter 1

General Introduction 
Physiological adaptations (i.e. hibernation, freezing, and estivation) to environmental changes is vital to the survival of many if not all organisms. This is especially true for organisms that face extreme environmental challenges, which have developed a range of adaptations to ensure their survival. One such adaptation used by some mammals in order to survive prolonged seasonal exposure to stressful environmental conditions such as lack of food, frigid temperatures, and so on, is hibernation. The thirteen-lined ground squirrel (Ictidomys tridecemlineatus) is an excellent example of a hibernating mammal as these animals are native to the central prairies of North America, and they survive winters by hibernating underground. In preparation for hibernation, ground squirrels enter a state of hyperphagia where excessive eating results in large increases in body weight by up to $40 \%$ (Storey, 2010). During hibernation, these animals undergo cycles of torpor and arousal. During torpor, the animals suppress their metabolic rate (often to just $2-4 \%$ of normal conditions) and drop their core $\mathrm{T}_{\mathrm{b}}$ from $35-38^{\circ} \mathrm{C}$ to levels that match the ambient temperature of its surroundings (as low as $0-5^{\circ} \mathrm{C}$ ) (Frerichs \& Hallenbeck, 1998; Storey \& Storey, 2004; Storey, 2010; Wang \& Lee, 2011). During the process of metabolic rate depression within torpor, most physiological functions are reduced; respiration rates (approximately $2.5 \%$ of euthermia), organ perfusion ( $<10 \%$ of euthermia), neuron firing, and a shift in the metabolic profile from a reliance on carbohydrate metabolism to fat metabolism via $\beta$-oxidation are such examples (Buck \& Barnes, 2000; McArthur \& Milsom, 1991; Storey \& Storey, 2004). Prolonged periods of torpor (often 1-2 weeks or more) are interspersed with brief periods of arousal where metabolic rate and $\mathrm{T}_{\mathrm{b}}$ return to euthermic levels.

This strategy for conserving energy can save hibernating ground squirrels up to $88 \%$ of the ATP expenditure that would otherwise be required to maintain euthermic physiological conditions over the winter months (Wang \& Lee, 2011). Metabolic rate suppression is a 
controlled process that maintains cellular homeostasis at colder $\mathrm{T}_{\mathrm{b}}$ while simultaneously reprioritizing ATP use by different cell functions. These functions include cell preservation strategies such as antioxidant defense, differential regulation of enzymes by mechanisms like reversible protein phosphorylation, differential regulation of protein chaperones, in addition to differential regulation of transcription factors that modulate the expression of selected genes and/or proteins that support cell- or tissue-specific needs (Fahlman, Storey, \& Storey, 2000; MacDonald \& Storey, 2005; Mamady \& Storey, 2006; Morin \& Storey, 2006; Morin, Ni, McMullen, \& Storey, 2008).

During hibernation, each organ/tissue of the hibernator must make specific adjustments that allow them to maintain or preserve physiological function under the periods of low $T_{b}$ during torpor, the fluctuations in $\mathrm{T}_{\mathrm{b}}$ as a result of torpor-arousal cycles, and various cellular stresses.

\section{Skeletal Muscle}

The skeletal muscle experiences muscle wasting, whereby reductions in muscle mass, strength, and the relative amount of slow oxidative fiber occur when a prolonged period of mechanical unloading or inactivity occurs (Bassel-Duby \& Olson, 2006; Choi, Selpides, Nowell, \& Rourke, 2009; Malatesta, Perdoni, Battistelli, Muller, \& Zancanaro, 2009; Rourke, Yokoyama, Milsom, \& Caiozzo, 2004). For hibernators, this muscle wasting would be highly disadvantageous, because following hibernation, these animals need to resume natural activities and scavenge for food right away. Therefore, there is a need to reduce significant losses in muscle mass during hibernation. Interestingly, these animals seem to be able to do just that as numerous studies have demonstrated a lack of significant muscle wasting during hibernation despite the prolonged periods of inactivity and mechanical unloading that occur during 
hibernation (Cotton \& Harlow, 2015; Gao et al., 2012; Xu et al., 2013). As shown in Figure 1.1, the relative ratio of muscle mass/body weight actually increases throughout hibernation in ground squirrels due to significant losses in body weight as well as an extremely effective mechanism of muscle preservation and remodeling that is unique to hibernators (Gao et al., 2012). As one would expect, nitrogen balance appears to be maintained throughout hibernation due to a balance between protein synthesis and breakdown in the skeletal muscle (Lee et al., 2012). In summary, hibernators have unique mechanisms of muscle remodeling and preservation in comparison with non-hibernating mammals such as humans. We set out to elucidate the molecular mechanisms underlying this process with the hope that our findings could become applicable to the identification of novel targets for the treatment of muscle wasting diseases such as Duchenne Muscular Dystrophy and Spinal Muscular Atrophy (Boyer et al., 2014; Haslett et al., 2003).

\section{Cardiac Muscle}

Another organ that must make significant adjustments during hibernation is the heart. During torpor, the squirrel's heart rate is strongly reduced, often from euthermic rates of 350-400 beats/min to just $5-10$ beats/min. These changes in heart rate, in addition to the increased viscosity of blood at low $\mathrm{T}_{\mathrm{b}}$, require significant changes in cardiovascular dynamics (Frerichs \& Hallenbeck, 1998; Frerichs, Kennedy, Sokoloff, \& Hallenbeck, 1994). The strength of each individual contraction must be significantly greater as a result of pressure and volume overloads, therefore cardiac hypertrophy is observed during torpor (Depre et al., 2006). In humans, cardiac hypertrophy is often characterized by significant cardiac fibrosis whereby collagen deposition occurs to stiffen cardiac chamber walls, reduce diastolic filling, and ultimately preventing the heart from pumping enough blood to meet bodily demands. This is a condition known as heart 
failure, and it is caused by abnormal metabolic, structural, and functional events occurring in the heart (Day, 2013; Hill and Olson, 2008). What is fascinating about the hearts of ground squirrels is that their cardiac dimensions (left ventricular mass, internal dimensions, and wall dimensions) are increased during hibernation in comparison with active, non-hibernating squirrels (Nelson \& Rourke, 2013) (Figure 1.2). This phenomenon suggests that ground squirrels have an efficient mechanism of cardiac remodeling, whereby it can undergo cardiac hypertrophy when necessary to maintain perfusion and then reverse this process after hibernation. The molecular basis behind this mechanism has yet to be discovered and understanding how this process occurs could provide novel insight into the development and treatment of maladaptive cardiac hypertrophy and heart failure.

\section{Objectives and Hypotheses}

Although metabolic rate depression in I. tridecemlineatus is characterized by a global suppression of most processes that cause ATP expenditure, including important functions such as transcription and translation, positive regulation of select genes and proteins still occur in order to ensure the animal's survival. As a matter of fact, various approaches of gene- and proteinscreening have identified various targets that are upregulated during hibernation (Li et al., 2013; Storey \& Storey, 2010). These targets include transcription factors (TFs) that play an integral part in regulating gene transcription. Since these TFs regulate the transcription of specific genes and groups of genes that play vital roles in the cell, I decided to focus on studying TFs that regulate the expression of genes/proteins that play important roles in muscle remodeling. Characterizing the expression and activity of these TFs and their downstream targets over the torpor-arousal cycle will improve upon our current knowledge of the molecular mechanisms underlying the unique physiological processes that occur in ground squirrel skeletal and cardiac 
muscles. Given the lack of cures for diseases and conditions such as Spinal Muscular Atrophy, Duchenne Muscular Dystrophy, and heart failure, there is a need to identify novel targets for therapeutic intervention and to improve our understanding of the mechanisms underlying muscle remodeling (Boyer et al., 2014; Day, 2013; Haslett et al., 2003). Therefore, these are the challenges that motivate me to study and compare these two different types of muscle and how the squirrel is able to naturally avoid physiological dysfunction in these tissues.

\section{Regulation of the nuclear factor of activated T cells (NFAT)}

Currently, there are many TFs that have been implicated in muscle remodeling and they seem to play vital roles in both hypertrophy and atrophy, these TFs include MEF2, MyoG, Foxo, and NFAT (Armand et al., 2008; Boyer et al., 2014; Day, 2013; Haslett et al., 2003). The NFATs in particular are a family of transcription factors that have been implicated in multiple aspects of skeletal muscle remodeling; including hypertrophy, fiber-type switching, and myogenesis (Armand et al., 2008; Delling et al., 2000; Hudson et al., 2014). For instance, NFATc2-null mice have defective myoblast fusion and myogenesis, resulting in fibers with reduced size and delayed repair in response to injury (Horsley et al., 2001). In cardiomyocytes, NFATs regulate cardiomyocyte atrophy, apoptosis, development, and growth (Li et al., 2013; Lin et al., 2009; Liu, Wilkins, Lee, Ichijo, \& Molkentin, 2006; Molkentin et al., 1998; Schubert et al., 2003). In fact, in another hibernating animal - the woodchuck (Mormota monax) - Li et al. (2013) used iTRAQ technology and mass spectrometry to identify that there is an upregulation in the NFAT pathway during hibernation in the hearts of these animals. Inactive NFATs are located in the cytoplasm and are heavily phosphorylated, and NFATs are activated and nuclear localization occurs via dephosphorylation by calcineurin (Park et al., 2000), a calmodulin-stimulated protein phosphatase, allowing NFATs to translocate to the nucleus and bind to transcriptional complexes 
of their target genes (Rusnak \& Mertz, 2000) (Figure 1.3). Therefore, the rationale behind why we decided to study this family of TFs specifically with regard to muscle remodeling during hibernation is due to its role as a master regulator of muscle remodeling and its identification as a potential target through proteomics studies.

Hypothesis 1: I. tridecemlineatus undergoes significant skeletal muscle remodeling (simultaneous muscle protein synthesis and breakdown) in order to avoid disuse-induced skeletal muscle wasting during torpor. The NFAT-calcineurin pathway will be activated by calcium signaling during torpor in order to upregulate muscle specific genes that play a role in muscle hypertrophy and/or maintenance.

Chapter 3 explores this hypothesis by examining the protein levels (via immunoblotting) of NFATs, their downstream muscle specific targets, and calcium signaling factors that are upstream of NFATs in ground squirrel skeletal muscle over the torpor-arousal cycle.

Furthermore, nuclear localization of NFATs and their binding activities (defined as the ability of transcription factors to bind to their conserved promoter sequence) were examined using DNAProtein Interaction (DPI) ELISA assays to get a sense of NFAT activity, which is more important than NFAT levels with regard to their role in regulating gene transcription. We found that the NFAT TFs showed increased binding activity that was initiated by increased levels of $\mathrm{Ca}^{2+}$ signaling proteins upon entering torpor. There were modest increases in NFAT protein levels as well during torpor, and as a result the expression of muscle specific proteins were highly upregulated during torpor. In addition, we also found that the cellular environment has an effect on NFAT-binding to DNA; specifically, temperature and calcium concentration both affect NFAT activity. 
Hypothesis 2: I. tridecemlineatus undergoes cardiac muscle remodeling in order to maintain cardiovascular function during torpor through cardiac hypertrophy and to avoid chronic maladaptive cardiac hypertrophy by reversing this process during arousal. The NFATcalcineurin pathway will be activated by calcium signaling during torpor in order to upregulate muscle specific genes that play a role in cardiac hypertrophy, and this molecular response should differ from those of skeletal muscle.

Chapter 4 explores this hypothesis by examining the protein levels (via immunoblotting) of NFATs, their downstream targets in cardiac muscle, and calcium signaling factors that are upstream of NFATs in ground squirrel skeletal muscle over the torpor-arousal cycle. There were modest increases in NFAT protein levels as well during torpor and downregulation during arousal, especially in NFATc2 and $\mathrm{c} 3$, which are the NFATs that are most important in regulating the expression of genes important for muscle growth and development (Armand et al., 2008; Delling et al., 2000; Lin et al., 2009). The decreases in NFATc2 and c3 protein levels corresponded with a decline in the expression of calcium signaling factors, indicating that calcium signaling regulation of NFATs may play a role in the reversal of cardiac hypertrophy. Furthermore, the expression of muscle specific proteins were highly upregulated only during early torpor, and the pattern of expression was different from that of skeletal muscle.

\section{Regulation of forkhead box $O$ (Foxo) and Myogenin (MyoG)}

Other sets of transcription factors that play key roles in the regulation of muscle remodeling include MyoG and the Foxo family of TFs (Moresi et al., 2010; Sandri et al., 2004; Stitt et al., 2004). Recent studies have begun to elucidate the molecular basis of both skeletal and cardiac muscle remodeling in hibernators, with findings indicating that the peroxisome proliferator-activated receptor $\gamma$ coactivator $1-\alpha(\mathrm{PGC}-1 \alpha)$ and NFATs are implicated in this 
process (Li et al., 2013; Xu et al., 2013; Zhang \& Storey, 2015). However, a current gap in knowledge exists around whether the molecular pathways of muscle atrophy and protein degradation are activated in skeletal and cardiac muscle during hibernation as a result of inactivity and cardiac hypertrophy. The main signaling pathway that controls muscle atrophy involves the Foxo as well as the MyoG TFs, and their regulation of the ubiquitin proteasome system (UPS) (Moresi et al., 2010; Sandri et al., 2004; Stitt et al., 2004). The UPS is an important mechanism for protein degradation, whereby substrates are ligated to ubiquitin via E3 ubiquitin ligases like MAFbx/atrogin-1 and MURF1, which target these substrates for degradation in the proteasome. These ligases have been studied extensively in relation to muscle atrophy as they have been shown to degrade muscle proteins like MHC as well as MLC-1 and -2 (Foletta, White, Larsen, Léger, \& Russell, 2011; Herrmann, Lerman, \& Lerman, 2007; Schiaffino, Dyar, Ciciliot, Blaauw, \& Sandri, 2013). Due to the importance of both MAFbx and MURF1 for muscle atrophy, common regulators were found for both ligases. The Foxo family of TFs were the first of such factors (Moresi et al., 2010; Sandri et al., 2004; Stitt et al., 2004). Then, MyoG, which was initially identified as a regulator of myogenesis, was shown to be a positive regulator of both E3 ligases as well; where the expression of MAFbx and MURF1, as well as muscle atrophy were attenuated in MyoG-null mice (Moresi et al., 2010). The mammalian Foxo family has four members: Foxo1, Foxo3a, Foxo4, and Foxo6, that are involved in various cellular processes in addition to muscle atrophy, such as antioxidant defense and apoptosis (Birkenkamp \& Coffer, 2003; Greer \& Brunet, 2005; Wu \& Storey, 2014). Foxo1, Foxo3a, and Foxo4 are all regulated by the Akt/protein kinase B (PKB) signaling pathway, which is activated by PI3K in the presence of insulin (Burgering and Eijkelenboom, 2013). Specifically, Akt blocks the function of all three Foxo proteins through phosphorylation at 
conserved residues that lead to cytoplasmic localization (Brunet et al., 1999; Matsuzaki, Ichino, Hayashi, Yamamoto, \& Kikkawa, 2005; Takaishi et al., 1999; Tang, Nuñez, Barr, \& Guan, 1999) (Figure 1.4). However, Foxo4 transcriptional activity has been shown to be regulated through a separate pathway as well, involving the Ras and Ral GTPases, as well (De Ruiter, Burgering, \& Bos, 2001; Essers et al., 2004; Kops et al., 1999; Van Den Berg et al., 2013) (Figure 1.5).

Hypothesis 3: It is hypothesized that during the avoidance of muscle loss during hibernation, muscle atrophy signaling pathways regulating the UPS will either be inhibited or be stable during torpor. It is expected that differential regulation of MAFbx and MURF1 expression through Foxo4 and MyoG will be observed, and that Foxo4 dephosphorylation through inhibition of the Ras-Ral pathway will play a role in this process.

Chapter 5 explores this hypothesis by examining the protein levels (via immunoblotting) of Foxo4, phosphorylated Foxo4 (p-Foxo4), MyoG, MAFbx, MURF1, as well as the main factors involved in the Ras-Ral pathway (Ras, Ral, Ralbp1), in ground squirrel skeletal muscle over the torpor-arousal cycle. Furthermore, nuclear localization of Foxo4 and its activity with regard to its ability to regulating gene transcription will be characterized by assessing the levels of phosphorylated Foxo4 at different residues. We identified that although there is an increase in Foxo4 protein levels during torpor, there is a greater decrease in activated Foxo4 (p-Foxo4 T451). Therefore, there is a decrease in Foxo4 activity during torpor, which is reflected by decreases in Ras, Ral, and Ralbp1 protein levels during torpor as well. This decrease in Foxo4 activity, coupled with decreases in MyoG protein levels during torpor, resulted in decreased expression in both MAFbx and MURF1 during torpor, especially at early torpor (ET). 
Hypothesis 4: It is predicted that I. tridecemlineatus avoids reverses cardiac hypertrophy by activating muscle atrophy signaling pathways as they are aroused from torpor. The Foxo family of transcription factors as well as MyoG are believed to play a significant role in this reversal of cardiac hypertrophy through their regulation of the UPS.

Chapter 6 explores this hypothesis by examining the protein levels of the Foxo family of transcription factors (Foxo1, 3a, 4) as well as their phosphorylated forms via immunoblotting. In addition, levels of MyoG and the ubiquitin ligases MAFbx and MURF were characterized as well. Foxo1 and 3a nuclear translocation was assessed by characterizing the p-Foxo levels as Akt/PKB phosphorylates Foxol and $3 \mathrm{a}$ at the residues tested in order to prevent nuclear translocation (Dobson et al., 2011). Immunoblotting results demonstrated that there is upregulation of Foxo1 and 3a protein levels as well as decreases in inactive, phosphorylated Foxo1 and 3a proteins during torpor in comparison with euthermic control. Foxo4 and MyoG on the other hand increased in late torpor. MAFbx and MURF1 showed a similar pattern of expression where their protein levels increased in late torpor as well as arousal, thus suggesting that the ubiquitin proteasome system (UPS) is activated as ground squirrel are aroused from torpor, which could be causing muscle atrophy and the reversal of cardiac hypertrophy.

\section{Figures}




\begin{tabular}{llllll}
\hline Group & $\begin{array}{l}\text { BW before } \\
\text { hibernation } \\
(\mathrm{g})\end{array}$ & $\begin{array}{l}\text { BW at } \\
\text { experiment } \\
\text { time }(\mathrm{g})\end{array}$ & $\begin{array}{l}\text { BW loss } \\
(\%)\end{array}$ & $\begin{array}{l}\text { EDL MWW at } \\
\text { experiment } \\
\text { time }(\mathrm{mg})\end{array}$ & $\begin{array}{l}\text { EDL MWW/BW } \\
\text { at experiment } \\
\text { time }(\mathrm{mg} / \mathrm{g})\end{array}$ \\
\hline Pre-H & $345 \pm 18$ & $345 \pm 18$ & 0.00 & $133 \pm 13$ & $0.39 \pm 0.05$ \\
$\mathrm{H} 1$ & $363 \pm 36$ & $315 \pm 30^{* * *}$ & $13.1 \pm 3.3$ & $127 \pm 11$ & $0.41 \pm 0.04$ \\
$\mathrm{H} 2$ & $333 \pm 31$ & $254 \pm 22^{* * *}$ & $23.5 \pm 6.1$ & $122 \pm 13$ & $0.48 \pm 0.04^{\# \#}$ \\
Post-H & $349 \pm 33$ & $220 \pm 19^{* * *}$ & $36.5 \pm 9.4$ & $116 \pm 15^{\#}$ & $0.53 \pm 0.06^{\# \#}$ \\
\hline
\end{tabular}

$\mathrm{BW}=$ body mass, $\mathrm{MWW}=$ muscle wet mass.

${ }^{\#} p<0.05$, \# $p<0.001$, compared to Pre-H (ANOVA-LSD); ${ }^{* * *} p<0.001$, compared to the BW of themselves before hibernation (paired samples $T$ test).

Figure 1.1: Ground squirrel body mass, muscle wet mass, and muscle-to-body weight ratio before, during, and after hibernation (mean $\pm \mathrm{SD}, \mathrm{N}=8$ each). $\mathrm{EDL}-$ Extensor Digitorum Longus. Adapted from (Gao et al., 2012).

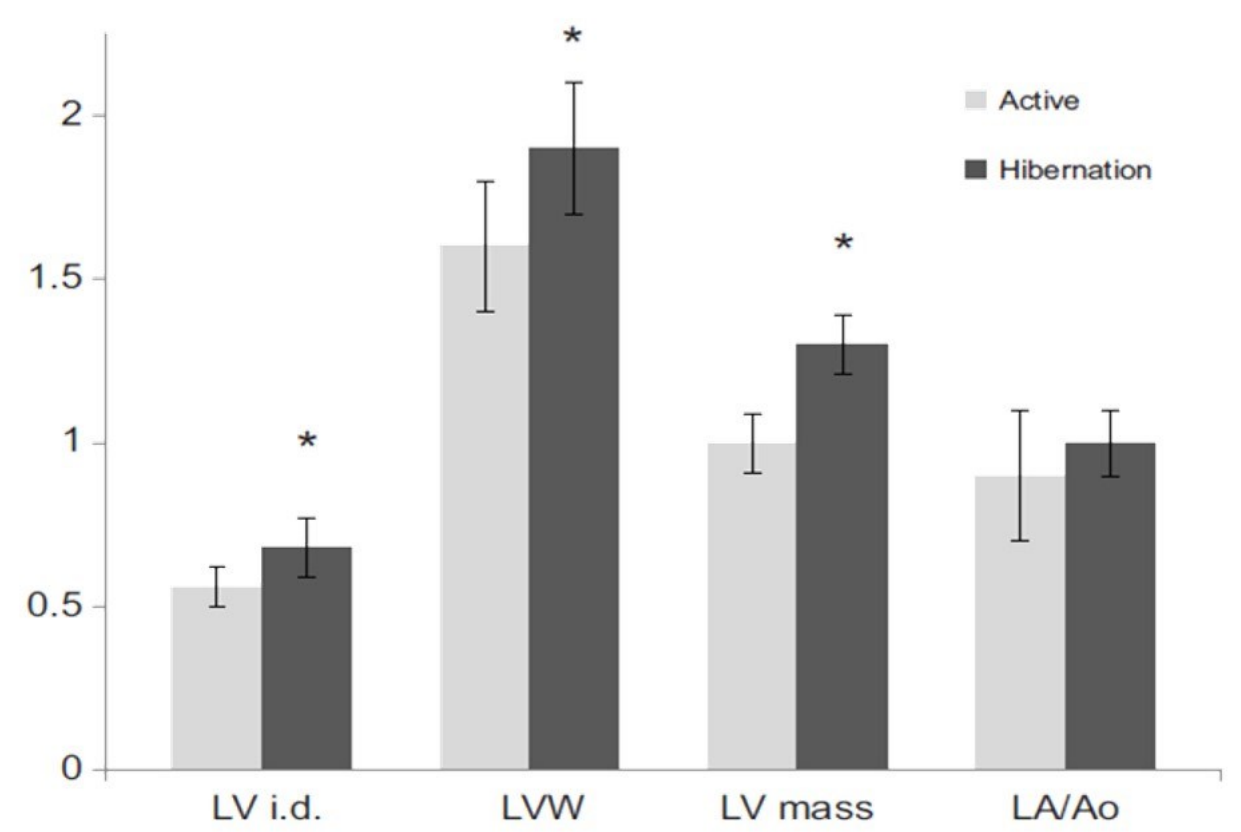

Figure 1.2: Left ventricular internal diameter dimension (LV i.d.), LV wall dimensions (LVW), LV mass, and left atrium (LA) to aortic (Ao) root dimension are greater in hibernation than control in squirrels. ${ }^{*} \mathrm{p}<0.05$. Adapted from (Nelson \& Rourke, 2013). 


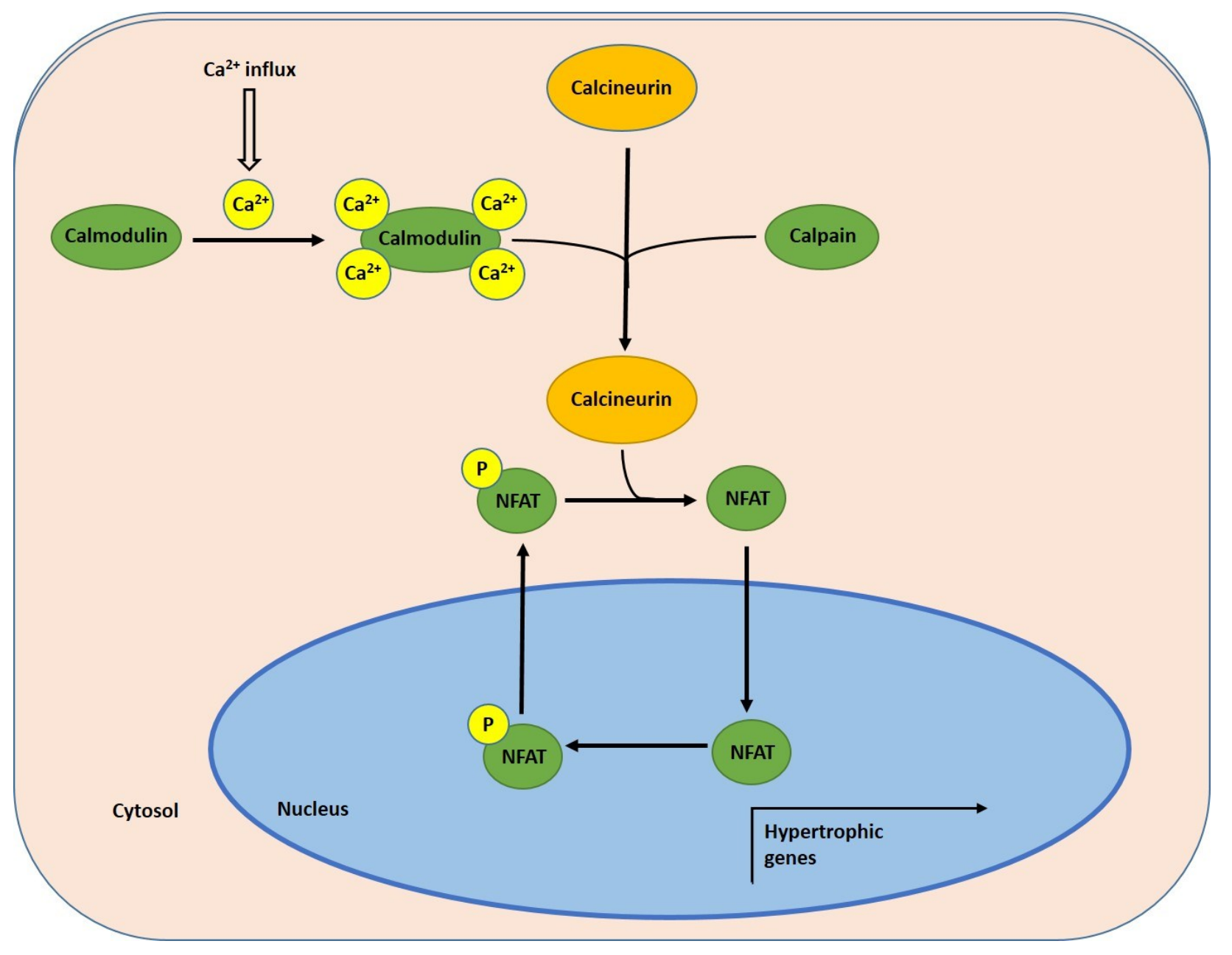

Figure 1.3: Schematic diagram of the calcineurin-NFAT pathway and its regulation by $\mathrm{Ca}^{2+}$ signaling in myocytes (muscle cells). $\mathrm{Ca}^{2+}$ uptake by myocytes activates calmodulin and calpain1, which activate calcineurin as a result. Activated calcineurin removes the phosphate group on NFAT transcription factors, allowing for nuclear translocation, where it can regulate the expression of genes essential for muscle hypertrophy. When intracellular $\mathrm{Ca}^{2+}$ levels decrease and calcineurin becomes inactive, and NFAT is phosphorylated and exported by several different kinases including GSK3 $\beta$, PKA, and Dyrk1a. 


\section{Kinase \\ Inhibitors}

Phosphorylation

Sites

Regulators

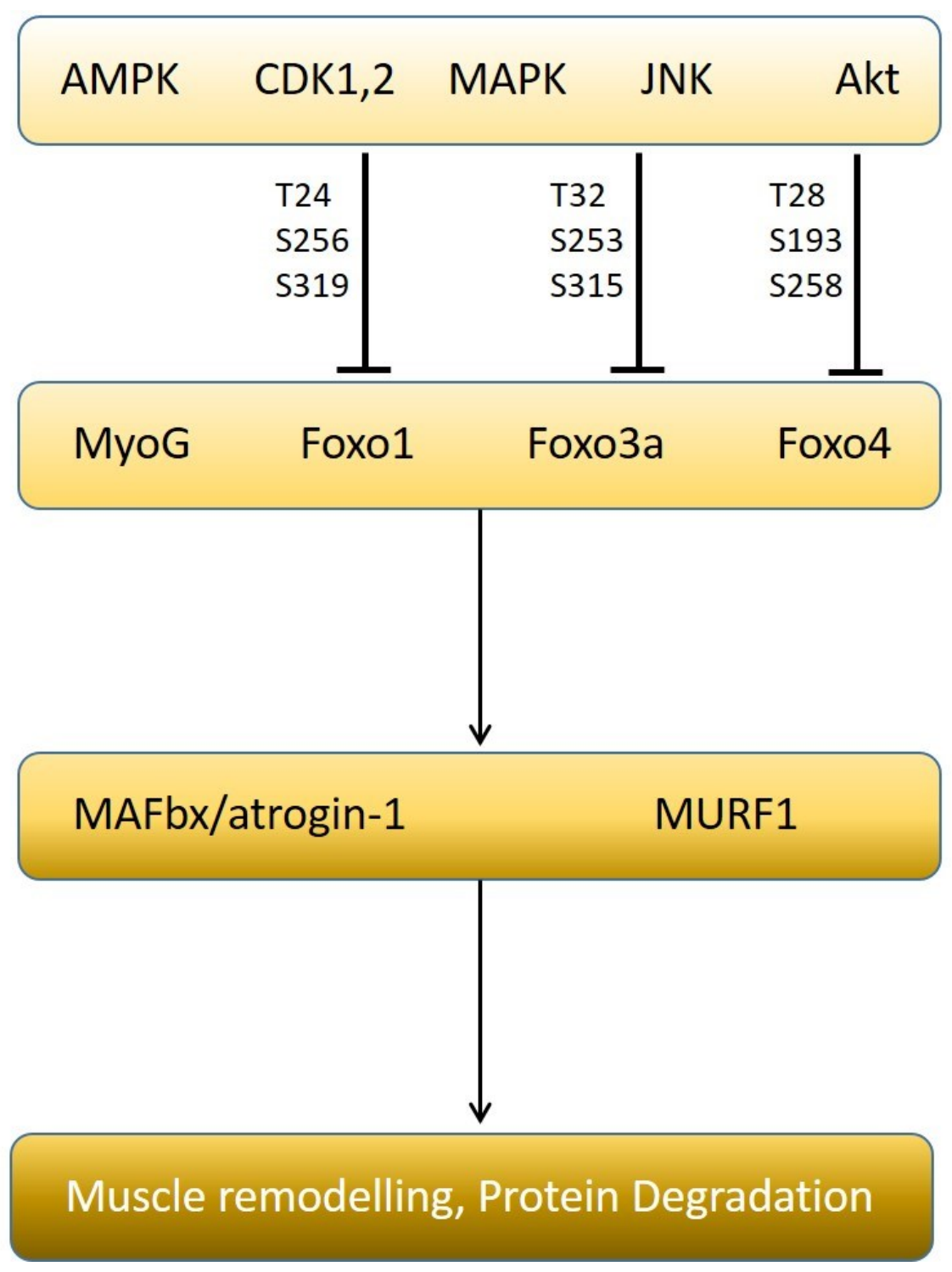

Figure 1.4: Signaling pathways involved in E3 ubiquitin ligase (MAFbx and MURF1) regulation which result in physiological outcomes such as muscle remodeling and protein degradation/atrophy in skeletal and heart muscle. Transcriptional regulation of E3 ligases occur via several transcription factors (TFs) such as Foxos and myogenin. Several kinases have the ability to inhibit Foxo activation and translocation to the nucleus through phosphorylation on several residues for Foxo1, 3a, and 4. 

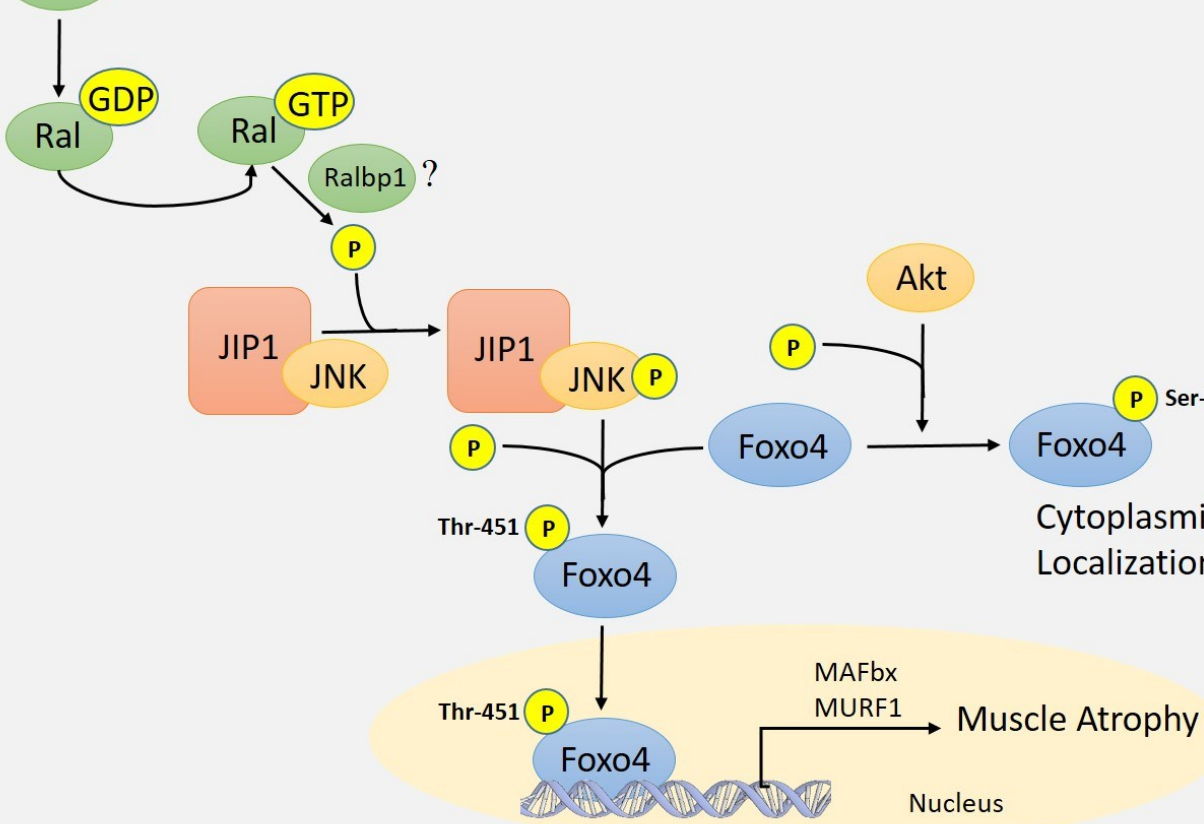

Figure 1.5: Model for Akt-dependent and Ras-Ral pathway-dependent FOXO4 regulation of muscle atrophy. Cellular stresses (i.e. hyperoxia, increase in $\mathrm{T}_{\mathrm{b}}$ ) that induce production of reactive oxygen species (ROS) results in activation of the Ras-Ral pathway. Activated RalA then regulates the assembly, activation, and phosphorylation of JNK onto the JIP1 scaffold, possibly involving Ralbp1, a protein commonly found in association with RalA. Phosphorylated JNK is able to phosphorylate and activate Foxo4 at Threonine 451 so that it can regulate the expression of E3 ubiquitin ligases like MAFbx/atrogin-1 and MURF1, thus promoting muscle atrophy. Phosphorylation of Foxo4 at Serine 197 by Akt results in cytoplasmic localization, thus preventing Foxo4 from translocating to the nucleus and regulating transcription. 
Chapter 2

Materials and Methods 


\section{Animals}

Thirteen-lined ground squirrels (I. tridecemlineatus), which weighed 150-300 g, were wild-captured by the United States Department of Agriculture (USDA) licensed trappers (TLS Research, Bloomingdale, IL). Animals were then transported to the Animal Hibernation Facility at the National Institute of Neurological Disorders and Stroke (NINDS, Bethesda, MD), where all experiments were conducted by the laboratory of Dr. J.M. Hallenbeck as previously described (McMullen \& Hallenbeck, 2010). All animal procedures were approved by the Animal Care and Use Committee of the National Institute of Neurological Disorders and Stroke (NIH; animal protocol no. ASP 1223-05). Male and female ground squirrels were sampled equally in the study with a mixture of genders in each experimental condition and all animals were between 1-3 years of age, although the exact age of the animals is unknown since animals were wild-captured. At NINDS, animals were housed individually in cages in a holding room with a constant ambient temperature of $21^{\circ} \mathrm{C}$ under a $12 \mathrm{~h}$ light: $12 \mathrm{~h}$ dark cycle. Animals were fitted with a sterile programmable temperature transponder (IPTT-300; Bio Medic Data Systems) injected subcutaneously in the intrascapular area while the squirrels were anaesthetized with $5 \%$ isofluorane. Animals were fed water and standard rodent chow ad libitum until they gained sufficient lipid stores to enter hibernation.

To enable a natural transition into torpor, animals were transferred to constant darkness in an environmental chamber at $4-5^{\circ} \mathrm{C}$ at the end of October. To not disturb the torpid squirrels, a red safe light (3-5 lux) was used when entering the chamber and a heavy dark curtain was used to shield the shelves containing the cages and block the light and sound resulting from opening and closing the door to the environmental chamber. Body temperature $\left(\mathrm{T}_{\mathrm{b}}\right)$, time elapsed, and 
respiration rates were monitored and used to determine the stage of torpor-arousal cycle. All animals had been through torpor-arousal bouts prior to sampling, therefore they were deep in hibernation when sampling took place. Four different animals were euthanized and tissue samples were collected at the following sampling points: 1) Euthermic Room temperature (ER); these animals were held in the holding room with an ambient temperature of $21^{\circ} \mathrm{C}\left(\mathrm{T}_{\mathrm{b}}=\sim 37^{\circ} \mathrm{C}\right)$. Tissues were collected from animals at this time point after they had reached their plateau weight. 2) EC designates euthermic in the cold room. These squirrels had a stable $\mathrm{T}_{\mathrm{b}}$ of $37^{\circ} \mathrm{C}$ for at least three days and were capable of entering torpor, but had not re-entered hibernation in the past $72 \mathrm{~h}$. These euthermic animals displayed slow-wave sleep characteristics that were observed in all sampling animals, and thus were chosen as the reference group to eliminate compounding variables of environmental light, temperature, feeding, in addition to time/season. 3) EN designates entrance into hibernation; entrance into the torpor-arousal cycle is characterized by falling $\mathrm{T}_{\mathrm{b}}$ with sampling occurring between $31^{\circ}$ and $18^{\circ} \mathrm{C} .4$ ) ET designates early torpor; squirrels had entered torpor with a stable $\mathrm{T}_{\mathrm{b}}$ at $5-8^{\circ} \mathrm{C}$ for $\sim 24 \mathrm{~h}$. 5) LT designates late torpor; animals maintained $a T_{b}$ at $5-8^{\circ} \mathrm{C}$ for $>5$ days. 6) EA designates early arousal; animals with a $T_{b}$ rising to at least $\sim 12^{\circ} \mathrm{C}$ with increasing respiration to at least 60 breaths/min after torpor, 7) LA designates late arousal; animals with increased respiration rate and $\mathrm{T}_{\mathrm{b}}$ of $28-32^{\circ} \mathrm{C} .8$ ) IA designates interbout arousal; animals were naturally aroused after the torpor phase of the hibernation bout and reached the respiratory rate, metabolic rate, and body temperature of fully aroused animals for 6 hours after being in torpor for at least 5 days. These animals remain in the hibernaculum $\left(4^{\circ} \mathrm{C}\right)$ but their core body temperature is back to $\sim 37^{\circ} \mathrm{C}$. The skeletal muscle was collected and used for analysis from a mixture of hind limb muscles whereas the cardiac muscle 
was collected from a mixture of atrial and ventricular tissue. A diagram of these torpor-arousal cycle stages is shown in Figure 2.1, which was adapted from Tessier \& Storey (2016).

\section{Total Protein Extract Preparation}

Total soluble protein extracts were prepared as previously described (Zhang \& Storey, 2015) for samples of frozen skeletal muscle and heart from 4 animals for each stage in the torpor-arousal cycle, where samples were collected from EC, EN, ET, LT, and EA for both tissues. LA and ER were collected only for skeletal muscle and IA was collected only for cardiac muscle due to limited tissue samples. Frozen samples of $\sim 0.5 \mathrm{~g}$ tissue were quickly weighed, powdered into small pieces under liquid nitrogen and then homogenized (using a Polytron PT10)1:3 w:v in ice-cold homogenizing buffer (20 mM Hepes, $200 \mathrm{mM} \mathrm{NaCl,} 0.1 \mathrm{mM}$ EDTA, $10 \mathrm{mM} \mathrm{NaF}, 1 \mathrm{mM} \mathrm{Na}_{3} \mathrm{VO}_{4}, 10 \mathrm{mM} \beta$-glycerophosphate at a $\mathrm{pH}$ of 7.5) with $1 \mathrm{mM}$ phenylmethylsulfonyl fluoride (Bioshop) and $1 \mu \mathrm{L} / \mathrm{mL}$ protease inhibitor cocktail (Bioshop) added. Samples were centrifuged at $10,000 \mathrm{rpm}$ for $10 \mathrm{~min}$ at $4^{\circ} \mathrm{C}$ and supernatants were removed. Soluble protein concentration was assayed using the BioRad reagent (BioRad Laboratories, Hercules, CA; Cat \#500-0006) at $595 \mathrm{~nm}$ on a MR5000 microplate reader. Samples were then adjusted to a final protein concentration of $10 \mu \mathrm{g} / \mu \mathrm{L}$ by the addition of a small volume of homogenizing buffer and then aliquots were combined 1:1 v:v with 2x SDS loading buffer (100 mM Tris-base, pH 6.8, 4\% w:v SDS, 20\% v:v glycerol, 0.2\% w:v bromophenol blue, 10\% $\mathrm{v}: \mathrm{v} 2$-mercaptoethanol) and then boiled. The final protein samples at a concentration of $5 \mu \mathrm{g} / \mu \mathrm{L}$ were stored at $-20^{\circ} \mathrm{C}$ until use. 


\section{Preparation of Nuclear Protein Extracts}

Nuclear protein extracts were prepared as previously described (Zhang \& Storey, 2015) and were separately extracted from the skeletal muscle of 4 animals for each of the seven experimental stages (ER, EC, EN, ET, LT, EA, LA). Frozen skeletal muscle samples were homogenized 1:2 w:v using a non-mechanical Dounce homogenizer (5 piston strokes) in lysis buffer (10 mM HEPES, pH 7.9, 10 mM KCl, 10 mM EDTA, 20 mM $\beta$-glycerophosphate), with $10 \mu \mathrm{L}$ of $100 \mathrm{mM}$ DTT, $10 \mu \mathrm{L}$ of protease inhibitor cocktail added immediately before homogenization. Samples were centrifuged for $10 \mathrm{~min}$ at $10,000 \mathrm{rpm}$ and $4^{\circ} \mathrm{C}$ and supernatants were removed as the cytoplasmic fraction. Pellets were resuspended in $147 \mu \mathrm{L}$ of nuclear extraction buffer (20mM HEPES, pH 7.9, 400mM NaCl, $1 \mathrm{mM}$ EDTA, 10\% v/v glycerol, 20mM $\beta$-glycerophosphate) with $1.5 \mu \mathrm{L}$ of $100 \mathrm{mM}$ DTT, and $1.5 \mu \mathrm{L}$ of protease inhibitor cocktail added. Samples were incubated on ice with gentle rocking for $1 \mathrm{~h}$ and then centrifuged for 10 min at $10,000 \mathrm{rpm}$ at $4^{\circ} \mathrm{C}$. Protein concentrations were determined with the Bio-Rad protein assay, adjusted to $5 \mu \mathrm{g} / \mu \mathrm{L}$, and samples were stored at $-80^{\circ} \mathrm{C}$ until use.

\section{Western Blotting}

The BioRad Mini Protean III system was used for SDS-PAGE. Equal amounts of protein from each sample (25 -35 $\mu \mathrm{g}$ depending on the protein tested) were loaded onto 6$15 \%$ polyacrylamide gels (depending on the protein tested) and were run at $180 \mathrm{~V}$ for $60-180$ min. Polyacrylamide gels were made based on a discontinuous gel system, which included the stacking gel at pH $6.8(130 \mu 11.0 \mathrm{M}$ Tris- $\mathrm{HCl}, 170 \mu \mathrm{l} 30 \%$ acrylamide, $680 \mu \mathrm{l}$ water, $10 \mu \mathrm{l} 10 \%$ SDS, $10 \mu 1$ 10\% APS, $1 \mu$ TEMED) and a resolving gel at pH 8.8 (1.3 ml 1.5 M Tris-base, 1.7 $\mathrm{ml} \mathrm{30 \%}$ acrylamide, $2.0 \mathrm{ml}$ water, $50 \mu \mathrm{l} 10 \%$ SDS, $50 \mu 1$ 10\% APS, $2 \mu 1$ TEMED, this is the 
recipe for a $10 \%$ gel). The running buffer was diluted 10 -fold from the stock solution ( $25.5 \mathrm{~g}$ Tris-base, $460 \mathrm{~g}$ glycine, $25 \mathrm{~g}$ SDS, adjusted to $2.5 \mathrm{~L}$ with water) before use. Proteins were then transferred to PVDF membranes by electroblotting at $160 \mathrm{~mA}$ for $60-180$ min depending on the protein tested or at $30 \mathrm{~V}$ for 100 min for small molecular weight proteins using a transfer buffer containing $25 \mathrm{mM}$ Tris (pH 8.5), $192 \mathrm{mM}$ glycine and 10\% v:v methanol at room temperature. Membranes were then blocked for 30 min with $2.5-10 \% \mathrm{w}: \mathrm{v}$ milk, depending on the protein tested, in 1x TBST (20 mM Tris base, $\mathrm{pH} 7.6,140 \mathrm{mM} \mathrm{NaCl}, 0.05 \% \mathrm{v}: \mathrm{v}$ Tween-20, 90\% v:v $\mathrm{ddH}_{2} \mathrm{O}$ ). After washing for $3 \times 5$ min again with $1 \times$ TBST, membranes were probed with specific primary antibodies at $4^{\circ} \mathrm{C}$ overnight at a concentration of 1:500-1:1000 depending on the protein tested. After probing with primary antibody, membranes were washed for 3 x 5 min with $1 \mathrm{x}$ TBST and then incubated with HRP-linked anti-rabbit or anti-goat IgG secondary antibody (Bioshop: 1:6000 v:v dilution) for $30 \mathrm{~min}$ at room temperature. After a second set of three washes, bands were visualized by enhanced chemiluminescence $\left(\mathrm{H}_{2} \mathrm{O}_{2}\right.$ and Luminol). Then, blots were stained using Coomassie blue $(0.25 \% \mathrm{w} / \mathrm{v}$ Coomassie brilliant blue, $7.5 \% \mathrm{v} / \mathrm{v}$ acetic acid, 50\% methanol) to visualize total protein levels. Immunoblot bands for ground squirrel proteins corresponded to the molecular weights indicated on the respective antibody specification sheets or the amino acid sequence of the I. tridecemlineatus isoform, as confirmed by running PINK Plus Prestained Protein Ladder (FroggaBio) or BLUeye Prestained Protein Ladder (FroggaBio) for high molecular weight proteins.

\section{DNA-Protein Interaction (DPI)-ELISA}

DNA oligonucleotides were designed based on the DNA binding elements of NFATc1-4 and were produced by Sigma Genosys (Oakville, ON, Canada). The biotinylated probe (NFAT 
5'-Biotin-GGGAAGGAAAGTGCGGGTGG-3') and the complement probe (NFAT 5'-Biotin CCACCCGCACCCTTTTTCCC-3') were first diluted in sterile water (500 pmol/ $\mu \mathrm{l})$, and the two probes were mixed 1:1 v:v for a total of $20 \mu 1$. Probes were then placed in a thermocycler for 10 min at $94^{\circ} \mathrm{C}$ and gradually cooled to room temperature. Double stranded DNA probes were diluted in 1x PBS (137 mM NaCl, $\left.2.7 \mathrm{mM} \mathrm{KCL}, 10 \mathrm{mM} \mathrm{Na}_{2} \mathrm{HPO}_{4}, \mathrm{pH} 7.4\right)$, and $50 \mu$ of diluted DNA probe was added (40 pmol DNA/well) to streptavidin-coated wells on a microplate. Following a $1 \mathrm{~h}$ incubation, unbound probe was discarded and wells were rinsed twice with $1 \mathrm{x}$ wash buffer (1X PBS containing 0.1\% Tween-20), and a third time with 1X PBS. Transcription factor binding buffer (10 mM HEPES, $50 \mathrm{mM} \mathrm{KCL}, 0.5 \mathrm{mM}$ EDTA, $3 \mathrm{mM} \mathrm{MgCl} 2,10 \% \mathrm{v} / \mathrm{v}$ glycerol, $0.5 \mathrm{mg} / \mathrm{ml}$ bovine serum albumin, $0.05 \%$ NP-40, $0.5 \mathrm{mM}$ DTT, $20 \mathrm{pg} / \mu \mathrm{l}$ Salmon Sperm DNA, $44 \mathrm{mM} \mathrm{NaCl}, \mathrm{pH} 7.9$ ) was added to each well containing the DNA probe along with 27.5 $\mu \mathrm{g}$ of the nuclear protein extract. Two negative control wells were loaded with transcription factor binding buffer but no protein. Following another $1 \mathrm{~h}$ incubation with gentle shaking, protein mixtures were discarded and the wells were washed three times with $1 \mathrm{x}$ wash buffer.

Diluted primary antibody (1:500) was then added (60 $\mu \mathrm{l} /$ well) for $1 \mathrm{~h}$ and were then discarded, and wells were rinsed three times with $1 \mathrm{x}$ wash buffer before incubation with diluted secondary $(1: 1000,60 \mu \mathrm{l} /$ well $)$ for $1 \mathrm{~h}$. This antibody was then discarded and wells were rinsed three times with wash buffer. After secondary antibody incubation and washing, bound antibody was detected using tetramethylbenzidine (TMB) (Bioshop). A $60 \mu$ aliquot of TMB was added to each well, colour was developed for 10-15 min, and then the reaction was stopped with $60 \mu \mathrm{l}$ of $1 \mathrm{M} \mathrm{HCl}$. Absorbance was measured at $450 \mathrm{~nm}$ (reference wavelength of $655 \mathrm{~nm}$ ) using a Multiskan spectrophotometer. To control for background absorbance and non-specific binding, test strip ELISA experiments were run with negative controls containing no probe or no protein 
or no primary antibody added being run in duplicates using a pooled sample of multiple sampling points. Conditions were optimized such that negative control wells showed $>50 \%$ decreases in absorbance relative to sample wells before quantification runs of sampling points were conducted.

\section{Environmental DPI-ELISA}

To assess how TF-DNA binding is altered when environmental conditions (temperature, $\left[\mathrm{CA}^{2+}\right]$, [urea]) are altered, the DPI-ELISA protocol described above was modified. To test for the effect of temperature on TF-DNA binding, the initial DNA probe synthesis, incubation, and washing steps were carried out as previously described. Afterwards, TF binding buffer was added to each well containing the DNA probe, plus one other well that is used to monitor solution temperature. Buffer temperature was monitored using a digital thermometer with two probes, one placed outside the solution to monitor ambient temperature and the other placed inside the well to monitor solution temperature. The ELISA plate was placed in either a $4^{\circ} \mathrm{C}$ fridge, a $37^{\circ} \mathrm{C}$ incubator, or left at room temperature. When the solution temperature has matched and stabilized to the ambient temperature inside the fridge or incubator, $27.5 \mu \mathrm{g}$ of nuclear extracts of EC and LT samples were added to wells containing the DNA probe with the exception of the duplicate negative controls, and the plates were placed on shakers. Following the one hour incubation, all plates were placed at room temperature and the rest of the procedure was performed as described above. Temperature DPI-ELISAs were performed to evaluate: 1) the effect of temperature $\left(37,21,4^{\circ} \mathrm{C}\right)$ on TF-DNA binding for EC samples, 2) the effect of temperature $\left(37,21,4^{\circ} \mathrm{C}\right)$ on binding for LT samples, and 3$)$ the difference in binding between EC and LT sampling points using their physiological temperatures, 37 and $4^{\circ} \mathrm{C}$, respectively. 
In order to test for the effect of $\mathrm{Ca}^{2+}$ or urea on TF-DNA binding, the DPI-ELISA protocol described above was followed; adjusting the TF binding buffer by adding $\mathrm{Ca}^{2+}$ or urea. To assess the effect of $\mathrm{Ca}^{2+}$ on TF-DNA binding, quantification runs were performed on four biological replicates of the LT samples with no protein and no $\mathrm{Ca}^{2+}$ (negative controls), no $\mathrm{Ca}^{2+}$, $100 \mathrm{n} \mathrm{Ca}^{2+}$, and $600 \mathrm{nM} \mathrm{Ca}^{2+}$ added to the TF binding buffer during the protein incubation step. $100 \mathrm{nM}$ and $600 \mathrm{nM}$ of $\mathrm{Ca}^{2+}$ were selected because they represent the minimum and maximum concentrations of nuclear $\mathrm{Ca}^{2+}$ that have been identified mathematically and experimentally (Brière, Xiong, Mazars, \& Ranjeva, 2006; Choi, Swanson, \& Gilroy, 2011; Dobi \& Agoston, 1998; Xiong, Tao, DePinho, \& Dong, 2012). A similar experiment was performed to assess for the effect of urea on TF-DNA binding, with quantification runs being conducted for LT samples $(\mathrm{n}=4)$ with no protein and no urea (negative controls), no urea, $5 \mathrm{mM}$ urea, and $100 \mathrm{mM}$ urea added to the transcription factor binding buffer during the protein incubation step. These two concentrations were tested as $5 \mathrm{mM}$ is approximately the normal physiological concentrations of serum urea in hibernating mammals (Chilian \& Tollefson, 1976; Kristofferson, 1963; Stenvinkel et al., 2013). Also, $100 \mathrm{mM}$ was shown experimentally as the maximum concentration of urea that could be supplemented to media before cell culture growth and survival was inhibited (Yancey \& Burg, 1990).

\section{Quantification and Statistics}

Band densities on chemiluminescent immunoblots were visualized using a Chemi-Genius BioImaging system (Syngene, Frederick, MD) and quantified using the Gene Tools software. Immunoblot band density in each lane was standardized against the summed intensity of a group of Coomassie-stained protein bands in the same lane; this group of bands was chosen because 
they were not located close to the protein band of interest but were prominent and constant across all samples. This method of standardizing against a total protein loading control has been suggested to be more accurate in comparison with standardizing against housekeeping proteins such as tubulin (Eaton et al., 2013). For DPI-ELISA experiments, quantification runs, absorbance readings were corrected by subtracting optical density (OD) values for each sampling point from OD values of blank wells containing no protein, and these values were then normalized relative to EC. Similarly, western blot band densities were also normalized at each other sampling point relative to EC. Immunoblotting and absorbance data are expressed as means \pm SEM, $\mathrm{n}=4$ independent samples from different animals. Statistical testing used the one-way ANOVA and the Tukey post-hoc functions from the GraphPad Prism software (San Diego, CA).

\section{Figures}

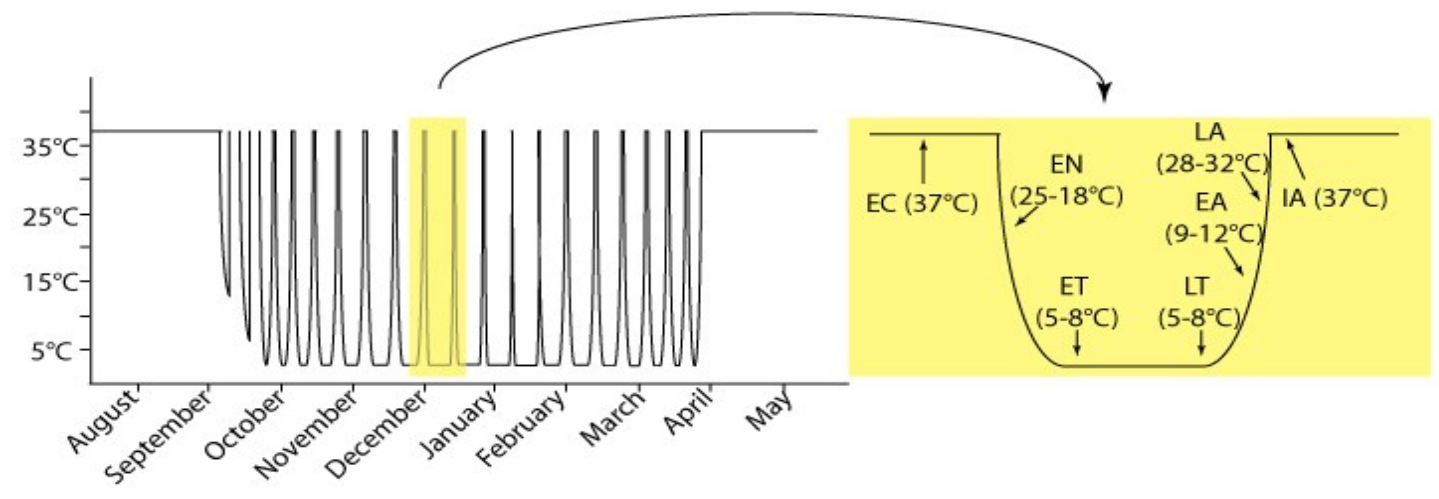

Figure 2.1: Schematic depiction of the hibernation bout indicating time points and body temperatures when animals were sacrificed. EC: euthermic in the cold room; EN: entrance into Torpor; ET: early Torpor; LT: late Torpor; EA: early Arousal; LA: late Arousal; IA: interbout arousal. Not shown, ER: euthermic at room temperature; this time point occurs before EC prehibernation and after IA post-hibernation. Total time between ET and LT is at least 5 days. Figure from Tessier \& Storey (2016). 


\section{Chapter 3}

Expression of nuclear factor of activated T cells and downstream muscle-specific proteins in ground squirrel skeletal muscle during

hibernation 


\section{Introduction}

The present chapter investigates how the skeletal muscle of thirteen-lined ground squirrels adapts on a molecular level to maintain function at low $\mathrm{T}_{\mathrm{b}}$ to support long-term torpor. As mentioned in chapter 1 , one potential issue affecting skeletal muscle during hibernation is disuse-induced muscle wasting, which occurs commonly after long periods of inactivity and results in reduced muscle mass, strength, and relative amount of slow oxidative muscle (BasselDuby \& Olson, 2006; Choi et al., 2009; Malatesta et al., 2009; Rourke et al., 2004). What makes hibernating mammals, specifically the thirteen-lined ground squirrel, interesting as a model to study muscle biology is that they demonstrate a lack of skeletal muscle atrophy despite prolonged periods of mechanical unloading that occur over long periods of hibernation (Cotton \& Harlow, 2015; Gao et al., 2012; Xu et al., 2013) (Figure 1.1).

The present chapter focuses on the roles and regulation of the nuclear factor of activated T cells (NFAT) family of transcription factors in I. tridecemlineatus, as they have been implicated as a key regulator of skeletal muscle hypertrophy (Armand et al., 2008; Delling et al., 2000; Hudson et al., 2014; Schiaffino, Sandri, \& Murgia, 2007; Zhang \& Storey, 2015). The NFAT family contains five members named NFAT1-5 or NFATc1-4 and NFAT5, with NFATc1-4 being regulated primarily by calcineurin (Rao, Luo, \& Hogan, 1997). Calcineurin is a CAM-stimulated protein phosphatase that regulates NFAT through dephosphorylation, thereby activating and allowing NFAT to translocate to the nucleus and regulate gene transcription (Rusnak \& Mertz, 2000). CAM is a ubiquitously expressed $\mathrm{Ca}^{2+}$-binding protein that is involved in a variety of signaling pathways that are $\mathrm{Ca}^{2+}$-dependent. It contains four EF-hand motifs, each of which binds a $\mathrm{Ca}^{2+}$ ion (Kretsinger, 1987). CAM regulates calcineurin by binding to the regulatory domain of the calcineurin A subunit when it is exposed due to conformational changes 
caused by activation of the calcineurin $\mathrm{B}$ subunit when there is an increase in intracellular $\mathrm{Ca}^{2+}$ levels (Klee, Crouch, \& Krinks, 1979; Yang \& Klee, 2000). When calcineurin B binds to $\mathrm{Ca}^{2+}$ ions, a conformational change occurs in its $\mathrm{C}$-terminal autoinhibitory domain, and the $\mathrm{Ca}^{2+}$ dependent cysteine protease calpain, specifically calpain $1 /$ calpain- $\mu$, cleaves the autoinhibitory domain, thus activating calcineurin (Burkard, 2005; Lee et al., 2014; Shioda, Moriguchi, Shirasaki, \& Fukunaga, 2006). Therefore, both calmodulin and calpain1 are important regulators of the NFAT-calcineurin pathway (Figure 1.3).

We sought to identify the role of $\mathrm{Ca}^{2+}$ signaling factors such as calmodulin, calpainl, and calcineurin on NFAT transcriptional regulation of muscle-specific proteins in the skeletal muscle of thirteen-lined ground squirrels. One such protein is myoferlin, a protein that is highly expressed in skeletal muscle and to a lesser degree in cardiac muscle (Davis, Delmonte, Ly, \& McNally, 2000). However, myoferlin is highly expressed specifically in myoblasts undergoing fusion, where it localizes at the sites of apposed membranes undergoing fusion (Davis, Doherty, Delmonte, \& McNally, 2002; Doherty et al., 2005). Furthermore, myoferlin mRNA was upregulated in human muscle affected by Duchenne muscular dystrophy (Haslett et al., 2003). Myoferlin contains multiple NFAT-binding sites in its promoter, which drove high levels of myoferlin expression in vitro and in vivo. Furthermore, expression was elevated in response to muscle damage (Demonbreun et al., 2010). In myoferlin-null mice, muscle fiber size was reduced due to impaired myoblast fusion, but the mice were still viable. However, myomaker, a membrane protein found in the muscle that also controls myoblast fusion results in the complete loss of myoblast fusion when it is mutated, resulting in the absence of all skeletal muscle, which leads to postnatal death in myomaker-null mice (Millay et al., 2013). In addition, myomaker expression and increased myoblast fusion has been shown to occur in adult satellite cells 
following muscle injury (Millay, Sutherland, Bassel-Duby, \& Olson, 2014). The regulation of myomaker is not well-understood due to its recent discovery, with only two MRFs, muscle differentiation protein $(\mathrm{MyoD})$ and myogenin $(\mathrm{MyoG})$, being known to induce myomaker transcription (Millay et al., 2014). However, NFAT has been shown to regulate the expression of MyoG cooperatively with MyoD (Armand et al., 2008).

In addition to affecting myomaker expression through NFATc2 regulation of MyoG, we also used a DNA-protein interaction (DPI) enzyme-linked immunosorbent assay (ELISA) to test the ability of NFATs to bind to a novel putative NFAT-binding sequence in the myomaker promoter. Since activation of the NFAT-calcineurin pathway ultimately leads to increased binding of NFAT transcription factors to its target promoters, we also used this technique to analyze transcription factor binding activity to DNA because of its simplicity (Brand et al., 2013; Brand, Kirchler, Hummel, Chaban, \& Wanke, 2010; Jagelska, Brázda, Pospisilová, Vojtesek, \& Palecek, 2002). Given the extreme environmental stressors confronting 13-lined ground squirrels during hibernation, we suspected that environmental factors such as temperature could potentially affect the binding ability of NFATs and potentially other transcription factors, to DNA. Recent literature has begun to show that gene expression could be affected by temperature, but no study has directly investigated the temperature dependence of transcription factor-binding to DNA (Novák et al., 2015; Chen, Nolte, \& Schlötterer, 2015; Riehle, Bennett, Lenski, \& Long, 2003; Swindell, Huebner, \& Weber, 2007). Most of these studies use DNA microarrays to study the global changes in gene expression when temperature stress is induced on an organism (Riehle et al., 2003; Swindell et al., 2007). However, although this approach identifies targets that may be involved in stress-response, it does not directly elucidate mechanisms such as transcription factor binding affinity. In addition to the ground squirrel's 
ability to thermoregulate during torpor-arousal cycles, they also show enhanced capabilities to maintaining intracellular $\mathrm{Ca}^{2+}$ and urea concentrations in comparison with non-hibernating animals under the same temperature stress (Chilian \& Tollefson, 1976; Kristofferson, 1963; Liu, Wang, \& Belke, 1991; Wang \& Zhou, 1999; Wang, Zhou, \& Qian, 1999; Wang, Lakatta, Cheng, \& Zhou, 2002). Therefore, we adapted our DPI-ELISA protocol in order to run these environmental ELISAs that allow us to characterize the effects of temperature and different cellular metabolites such as $\mathrm{Ca}^{2+}$, and urea on transcription factor-DNA binding.

To explore the role of calcium signaling in activating the NFAT-calcineurin pathway and their downstream muscle-specific targets, we quantified relative protein levels via immunoblotting and utilized the DPI-ELISA technique to measure changes in TF-binding to an oligonucleotide containing the NFAT response element. We predicted that the NFAT-calcineurin pathway would be activated through upregulation of $\mathrm{Ca}^{2+}$ signaling proteins during torpor, and that this would cause an upregulation of the muscle proteins, myoferlin and myomaker. The secondary objective of this study was to identify the impact of environmental conditions on NFAT-binding to target genes. We tested this theory using a modified, environmental DPIELISA that allowed us to adjust the temperature and concentration of metabolites within the assay.

\section{Materials and Methods}

\section{Animals Experimental Conditions}

All animals were captured, treated and their tissues were harvested following the same protocol as previously described in Chapter 2. The skeletal muscle used was a mixture of several hind limb muscles. 


\section{Total Protein and Nuclear Protein Extract Preparations}

Total soluble protein extracts and nuclear protein extracts were prepared from frozen hind leg skeletal muscle (approximately $500 \mathrm{mg}$ ) as previously described. Total protein extracts were used for western blotting and nuclear protein extracts were used for DPI-ELISA experiments as previously described in Chapter 2.

\section{Western Blotting}

Western blotting was performed as described in Chapter 2 for seven time points (ER, EC, EN, ET, LT, EA, LA) for NFATc1-4, myoferlin, and myomaker and for six time points (EC, EN, ET, LT, EA, LA) for calcineurin, CAM, and calpain1. Equal amounts of protein from each sample (25 $\mu \mathrm{g}$ ) were loaded onto $6 \%$ (NFATc1-4, myoferlin), $8 \%$ (calcineurin, calpain 1 ), or $15 \%$ (myomaker, CAM) polyacrylamide gels and were run at $180 \mathrm{~V}$ for 60-120 min. For calmodulin, $35 \mu \mathrm{g}$ of protein for each sample were loaded on the polyacrylamide gels. Proteins were then transferred to PVDF membranes by electroblotting at $320 \mathrm{~mA}$ for $60 \mathrm{~min}$ (myomaker), $90 \mathrm{~min}$ (calcineurin, calpain1), $120 \mathrm{~min}$ (NFATc1-3, myoferlin), or at $30 \mathrm{~V}$ for $100 \mathrm{~min}$ (calmodulin). Membranes incubated in primary antibodies were blocked for 30 min with $2.5 \%$ (for NFATc1-4) or 5\% (for CnA, CAM, calpain1, myoferlin and myomaker) w:v milk in 1x TBST.

Antibodies specific for mammalian NFAT-c1 (sc-13033), c2 (sc-13024), c3 (sc-8321), c4 (sc-13036), myoferlin (sc-134798), and myomaker (also known as TMEM8c, sc-244460) were purchased from Santa Cruz Biotechnologies. All antibodies were used at a 1:500 v:v dilution in 1x TBST. A calmodulin (06-396) antibody from Upstate Biotechnology (Lake Placid, NY), as well as calcineurin A (GTX111039) and calpain1 (GTX102340) antibodies from Genetex (Irving, CA) were purchased and used at a 1:1000 v:v dilution in 1x TBST. All primary antibody 
incubations took place over one night. Membranes that had been probed with myomaker (TMEM8c) were incubated with HRP-linked anti-goat IgG secondary antibody (BioShop: 1:6000 v:v dilution). All other antibodies were detected using HRP-linked anti-rabbit IgG secondary antibody (Bioshop: 1:6000 v:v dilution). All secondary antibody incubations were 30 minutes.

The primary antibodies cross-reacted with a single band on immunoblots at the expected molecular masses from the antibody specification sheets. Other parts of the procedure were carried out in accordance with the protocol described in Chapter 2.

\section{DPI-ELISA and Environmental DPI-ELISA}

DPI-ELISAs were performed as described in Chapter 2 for seven time points (ER, EC, EN, ET, LT, EA, LA) for NFATc1-4. Environmental ELISAs were performed for NFATc1, c3, and $\mathrm{c} 4$ using both the $\mathrm{EC}$ and LT time points to test for changes due to temperature $\left(37,21,4^{\circ} \mathrm{C}\right)$, and changes due to changes in $\left[\mathrm{Ca}^{2}\right]$ and [urea] were tested using the LT time point. Probes for NFATc1-4 were purchased from Sigma Genosys including both the biotinylated probe (NFAT 5'-Biotin-GGGAAGGAAAGTGCGGGTGG-3') and the complement probe (NFAT 5'-Biotin CCACCCGCACCCTTTTTCCC-3'). These probes were designed using comparative sequence analysis that identified conserved NFAT-binding sequences within the promoter of the myomaker gene. Specifically, the total nucleotide sequences of human (Accession number: NM_001080483.2), mouse (Accession number: NM_025376.3), and 13-lined ground squirrel (Accession number: XM_005336956.1) myomaker were accessed from the NCBI Nucleotide database and the sequence within 1500 base pairs (bp) upstream of the transcriptional start site was downloaded. These upstream sequences were compared using the Vista program 
(http://genome.lbl.gov/vista) with a window size of $100 \mathrm{bp}$ and a minimum sequence identity of 70\%. NFAT transcription factor binding sites were identified using the rVISTA program.

Predictions were made based on the TRANSFAC Professional 9.3 library using the default core similarity value of 0.75 and the matrix similarity value of 0.70 as previously described (Demonbreun et al., 2010).

Using rVista, all NFAT-DNA binding sites were identified and pairwise alignments were conducted between myomaker upstream sequences in squirrel and mouse (Figure 3.1A), and in squirrel and human (Figure 3.1B). The program displayed red lines identifying potential NFAT binding sites that were aligned, allowing a maximum core shift of $6 \mathrm{bp}$ and only one gap of any length inside it. Squirrel-mouse and squirrel-human alignments had two NFAT binding sites in common at 1095 and $~ 1440$-bp upstream of the myomaker transcriptional start site, shown on Figure 3.1 by the red lines. These two aligned NFAT binding sites were cross-referenced using the conserved NFAT binding sequence from the literature (GGAAA) (Hung, Wang, Chang, \& Shyu, 2008; Rao et al., 1997). Only the 1095-bp upstream sequence remained a probable NFATmyomaker binding site (Figure 3.1C). Subsequently, a DNA oligonucleotide was designed for this putative binding site (NFAT 5'-Biotin-GGGAAGGAAAGTGCGGGTGG-3') containing the general NFAT binding sequence and the flanking region specific to the myomaker promoter.

Findings indicated that NFATc1, c3, and c4 were able to bind specifically to the DNA oligonucleotide, as NFATc2 did not pass the test strip stage, with negative control wells showing high absorbance readings despite persistent optimization. The NFAT timecourse ELISA quantification runs as well as environmental ELISA quantification runs for NFATc1, c3, and c4 utilized the following conditions; $40 \mathrm{pmol} \mathrm{DNA} / \mathrm{well}, 27.5 \mu \mathrm{g}$ of protein/well, $1 \mu \mathrm{g}$ of salmon sperm/well, $44 \mathrm{mM} \mathrm{NaCl}, 1: 1000 \mathrm{v} / \mathrm{v}$ NFATc1, $\mathrm{c} 3$, and $\mathrm{c} 4$ primary antibody (same as those used 
for western blotting) in 1x PBST, 1:1000 secondary antibody (same as those used for western blotting) in 1x PBST. The rest of the protocol was followed as described in Chapter 2.

\section{Quantification and Statistics}

Band densities for NFATc1-4, myoferlin, myomaker, calcineurin, CAM, and calpain-1 on chemiluminescent immunoblots were visualized and quantified as described in Chapter 2. Relative binding affinity for the DPI-ELISA was quantified as described in Chapter 2. Data are expressed as means \pm SEM, $\mathrm{n}=4$ independent samples.

\section{Results}

Analysis of NFATc1-4 protein levels in skeletal muscle

NFATc1 protein levels decreased during torpor, reaching its lowest levels at LT (78\% lower in comparison with EC, $\mathrm{p}<0.05$ ), before rising back up to EC levels during arousal. NFATc2 levels on the other hand increased during torpor, peaking at LT (1.75 fold higher in comparison with EN, $\mathrm{p}<0.05)$. NFATc3 levels were highest initially at ER (1.82 fold higher in comparison with EC, $\mathrm{p}<0.05$ ) before decreasing dramatically. Protein levels then decreased again during LA (55\% lower in comparison with LT). Finally, NFATc4 levels were highest at ER, then levels rose from EC throughout the torpor-arousal cycle, peaking at LA (higher than EN by 1.97 fold, $\mathrm{p}<0.05$ ) (Figure 3.2).

Analysis of myoferlin and myomaker (TMEM8c) protein levels

Myoferlin levels increased dramatically during torpor (EN, ET, LT increased by 3.45, 4.75 , and 4.46 fold, respectively in comparison with $\mathrm{EC}, \mathrm{p}<0.05$ ) and then decreased upon 
entering arousal. Myomaker on the other hand showed constant protein levels from throughout the torpor-arousal cycle (Figure 3.3).

\section{Calcineurin, Calmodulin, and Calpain Protein Levels}

Calcineurin protein levels increased upon entering torpor (EN) by 1.19-fold in comparison with euthermic control (EC), $\mathrm{p}<0.05$. Protein levels then returned to baseline levels at EC during early torpor (ET), but it spiked once more by 2.08 -fold (in comparison with EC, $\mathrm{p}<0.05$ ) and reached its highest level during late torpor (LT). Upon entering arousal, calcineurin levels decreased once again to baseline at early arousal (EA) and then increased once more by 1.2-fold (compared to EC, $\mathrm{p}<0.05$ ) at late arousal (LA). Calpain1 showed a similar pattern of expression, where spikes in protein levels occurred at EN, LT, and LA. Calpain levels increased modestly at EN by 0.72 -fold relative to EC. At LT, there was a greater increase of 2.37 -fold relative to EC $(\mathrm{p}<0.05)$. The final spike at LA was even greater, where protein levels increased by 4.4-fold in comparison with EC ( $\mathrm{p}<0.05)$. Calmodulin protein levels remained fairly constant throughout the torpor-arousal cycle with the exception of EN, where levels increased by 2-fold relative to $\mathrm{EC}(\mathrm{p}<0.05)$ (Figure 3.4).

Analysis of NFATc1-4 Relative Binding to DNA

Relative binding to DNA (transcription factor activity) was measured for seven time points: ER, EC, EN, ET, LT, EA, and LA. NFATc1 activity decreased by $58 \%$ and $54 \%$ at EN and EA, respectively, relative to $\mathrm{ER}(\mathrm{p}<0.05)$, but there was a progressive increase throughout torpor from EN to LT. These decreases in DNA binding at EN and EA were seen in NFATc3 as well ( $46 \%$ and $30 \%$ lower respectively relative to EC). In addition, there was a large increase in NFATc3-DNA binding at LT (3.99-increase relative to EN, $\mathrm{p}<0.05)$. NFATc4 binding levels 
decreased modestly by $63 \%$ at $\mathrm{EN}$ from EC, then binding increased dramatically by 3.96 -fold relative at ET relative to EN ( $<<0.05)$. Following ET, binding activity decreased slightly at LT, then it increased slowly during EA and LA, with binding at LA being 3.77-fold greater than EN $(\mathrm{p}<0.05)$.

Effect of Temperature on NFATc1, c3, and c4 Relative-Binding to DNA

Due to the drastic changes in $\mathrm{T}_{\mathrm{b}}$ when ground squirrels enter torpor, we modified the DPI-ELISA in order to study the effect of temperature on NFATc1, c3, and c4 transcription factor binding to DNA. Three temperatures $\left(37\right.$, room temperature -21 , and $\left.4^{\circ} \mathrm{C}\right)$ were studied at the EC and LT sampling points. As mentioned previously, EC animals had not entered hibernation yet, so their $\mathrm{T}_{\mathrm{b}}$ remained at $37^{\circ} \mathrm{C}$. $\mathrm{LT}$ animals were at the deepest part of torpor; where $\mathrm{T}_{\mathrm{b}}$ was $4-5^{\circ} \mathrm{C}$ (McMullen \& Hallenbeck, 2010).

The temperature DPI-ELISA experiments performed on the EC time point showed that NFATc 1 and NFATc4 binding to DNA decreased dramatically ( $\mathrm{p}<0.05$ ) by $77 \%$ and $94 \%$, respectively, from $37^{\circ} \mathrm{C}$ to room temperature. NFATc3 on the other hand, showed a modest decrease of $37 \%$ in binding activity. However, when we compared changes in binding for all three NFATs between 37 and $4^{\circ} \mathrm{C}$, they all showed significant decreases in binding $(\mathrm{p}<0.05)$ by at least $84 \%$ (Figure 3.6A). Therefore, all three NFATs showed progressive declines in binding activity at the EC time point as temperature was decreased. The temperature DPI-ELISA using the LT sampling point showed a similar pattern for NFATc1 and $\mathrm{c} 4$, where binding decreased by $66 \%$ and $95 \%$, respectively, from $37^{\circ} \mathrm{C}$ to room temperature (Figure 3.6B). NFATc1 binding levels decreased further from $37^{\circ} \mathrm{C}$ to $4^{\circ} \mathrm{C}$ by $86 \%(\mathrm{p}<0.05)$. On the other hand, NFATc4 binding levels stabilized from room temperature to $4^{\circ} \mathrm{C}$. For the LT sampling point, NFATc 3 binding levels did not seem to be affected much by the changes in temperature, as there was only a 
modest decline in binding by $46 \%$ when comparing 37 to $4^{\circ} \mathrm{C}$ (Figure 3.6B). While analyzing the difference in transcription factor binding to DNA at physiological conditions from $\mathrm{EC}$ at $37^{\circ} \mathrm{C}$ to LT at $4^{\circ} \mathrm{C}$, we observed sharp declines in binding for both NFATc1 and c4 by $89 \%$ and $93 \%$, respectively $(\mathrm{p}<0.05)$. The decline in binding from EC to LT for NFATc3 was $64 \%$, which is less compared to the differences observed for NFATc 1 and $\mathrm{c} 4$, but this difference was still significant $(\mathrm{p}<0.05)$ (Figure 3.6C).

Effect of $\mathrm{Ca}^{2+}$ and Urea on NFATc1, c3, and c4 Relative-Binding to DNA

We tested for the effect of adding $\mathrm{Ca}^{2+}$ and urea to the DPI-ELISA assay in an attempt to discover how these two metabolites/substrates effect NFATc1, c3, and c4 TF-DNA binding. Urea and $\mathrm{Ca}^{2+}$ are of particular interest due to the unique changes in the animal's regulation of the urea cycle and $\mathrm{Ca}^{2+}$ signaling, which occur during mammalian hibernation (Chilian \& Tollefson, 1976; Epperson et al., 2011; Lee, Buck, Barnes, \& O'Brien, 2012; Stenvinkel et al., 2013; Wang et al., 1999; Wang et al., 2002). The addition of 5mM and 100mM of urea seemed to have no effect on the binding of NFATc1, $\mathrm{c} 3$, or $\mathrm{c} 4$ to DNA, with binding levels remaining stable throughout the different conditions during torpor (Figure 3.7A). When $100 \mathrm{nM}$ of $\mathrm{Ca}^{2+}$ was added to the protein incubation, the binding of NFATc1 to DNA showed a sizeable difference ( $42 \%$ decrease relative to the no $\mathrm{Ca}^{2+}$ control) out of the three NFATs tested at LT. When $600 \mathrm{nM}$ of $\mathrm{Ca}^{2+}$ was added however, NFATc1-DNA binding continued to decrease (57\% decrease relative to control, $\mathrm{p}<0.05$ ) with NFATc4 binding showing a 0.42 -fold increase relative to the no $\mathrm{Ca}^{2+}$ control. Throughout the conditions, NFATc3 binding to DNA did not change appreciably during torpor (Figure 3.7B).

\section{Discussion}


The present study aimed at furthering our understanding of the molecular mechanisms underlying muscle remodeling in both skeletal and cardiac muscle during hibernation in the 13lined ground squirrel. The ground squirrel provides an excellent natural model system for studying muscle remodeling as the skeletal muscle appears to undergo alternating patterns of atrophy and hypertrophy without a net loss in muscle mass and the cardiac muscle is known to undergo reversible cardiac hypertrophy (Hindle et al., 2014; Li et al., 2013; Nelson \& Rourke, 2013; Wickler, Hoyt, \& van Breukelen, 1991). However, the molecular mechanisms that are responsible the preservation and/or change of muscle structure/function during hibernation are not well known. Therefore, the present study focuses on the family of transcription factors known as NFATs, which have been shown to regulate targets associated with skeletal muscle hypertrophy, apoptosis, and development (Armand et al., 2008; Delling et al., 2000; Hudson et al., 2014; Li et al., 2013; Lin et al., 2009; Liu et al., 2006; Molkentin et al., 1998; Schiaffino et al., 2007; Schubert et al., 2003; Tessier \& Storey, 2012, 2010). Furthermore, the downstream targets myoferlin and myomaker were evaluated because of their newly identified and crucial role as muscle membrane proteins in muscle development and repair (Demonbreun et al., 2010; Doherty et al., 2005; Millay et al., 2013, 2014).

The relationship between myoferlin and NFATc1-4 was established when it was observed that the myoferlin promoter contains multiple NFAT-binding sites that were sufficient to drive high levels of myoferlin expression, especially following muscle damage (Demonbreun et al., 2010). However, the link between myomaker and NFAT remains more of a mystery. Currently, only MyoD and MyoG have been shown to induce myomaker transcription due to its very recent discovery (Millay et al., 2014). However, MyoD is a common co-factor of NFAT as they can bind to the same transcriptional complex, and they have been shown to synergistically 
regulate the transcription of myog (Armand et al., 2008). Comparative sequence analysis of the myomaker promoter region for potential NFAT transcription factor binding domains resulted in the identification of multiple sites with the consensus NFAT-binding sequence (GGAAA) from literature (Hung et al., 2008; Rao et al., 1997). Following further diagnostic analysis of potential NFAT-binding sites using the rVista alignment tool, one NFAT-binding domain 1095-bp upstream of the myomaker transcriptional start site was identified as it contained the consensus sequence in addition to showing conservation in squirrel-mouse and squirrel-human pairwise alignments (Figure 3.1). Therefore, comparative sequence analysis of the myomaker promoter region has resulted in the identification of a possible novel NFAT-myomaker binding site. Using DNA-Protein binding (DPI)-ELISAs, NFATc1, c3, and c4 were then shown to bind to this site differentially over the torpor-arousal cycle (Figure 3.5). DPI-ELISAs represent a simple and efficient way to measure transcription factor binding activity by quantitatively measuring the ability of transcription factors to bind to DNA from nuclear extracts. This is the first time that NFAT binding to the myomaker promoter has ever been identified, and this novel finding could lead to further studies that characterize NFAT regulation of this vital myogenic protein.

NFATs are regulated by calcineurin, a calmodulin-stimulated phosphatase that dephosphorylates NFATs, thus allowing them to translocate into the nucleus (Rusnak \& Mertz, 2000). Calcineurin is sensitive to changes in calcium levels and it is positively regulated by other calcium-signalling proteins such as calmodulin (CaM) and calpain (Al-Shanti \& Stewart, 2009; Burkard, 2005; Lee et al., 2014; Shibasaki, Hallin, \& Uchino, 2002; Shioda et al., 2006; Wu et al., 2004; Yang \& Klee, 2000). Therefore, experiments were conducted to study the role of calcineurin, CAM, and calpain as upstream regulators of the NFAT pathway within the context of muscle remodeling and maintenance. Due to the improved $\mathrm{Ca}^{2+}$-handling abilities of ground 
squirrels in comparison with non-hibernating mammals, $\left[\mathrm{Ca}^{2+}\right]$ changes very little from euthermia to the $0-5^{\circ} \mathrm{C} \mathrm{T}_{\mathrm{b}}$ seen during torpor (Frerichs \& Hallenbeck, 1998; Liu et al., 1991; Wang \& Lee, 2011; Wang \& Zhou, 1999; Wang et al., 1999; Wang et al., 2002). However, the amplitude of $\mathrm{Ca}^{2+}$ transients following excitation is actually increased following excitation at low temperatures, and as a result, stronger contractions with higher amplitudes are seen at lower temperatures (Liu, Wang, \& Belke, 1993; Liu, Wohlfart, \& Johansson, 1990; Wang, Zhou, \& Qian, 2000; Wang, Huang, Liu, \& Zhou, 1997). Therefore, greater spikes in intracellular $\left[\mathrm{Ca}^{2+}\right]$ following an action potential leads to an activation of NFAT-calcineurin pathway, allowing for a maintenance of muscle mass during hibernation.

Our results show that indeed, there is an upregulation of $\mathrm{Ca}^{2+}$ signaling proteins like calmodulin and calpain 1 during torpor, where protein levels increased by 2 -fold and 0.72 -fold relative to EC, respectively, at EN. These increases are accompanied by a 1.19-fold rise (compared to EC) in calcineurin levels downstream (Figure 3.4). As a result of this increase in calcineurin levels and activity, NFATc1, c3, and $\mathrm{c} 4$ translocate to the nucleus and show increases in binding to DNA during torpor (Figure 3.5). A similar pattern is seen during LA, where calpain1 levels increased by 4.4 -fold relative to EC and there was an accompanying increase in calcineurin levels as well (1.2-fold compared to EC) (Figure 3.4). Once more, there was an increase in NFATc1, c3, and c4 binding activity accompanying the upregulation and activation of calcineurin at LA (Figure 3.5). This upregulation of the NFAT-calcineurin pathway during LA is somewhat unexpected and could reflect the role that NFAT TFs play in not only muscle remodeling, but the generation of reactive oxygen species, which are produced rapidly due to oxidative thermogenesis in squirrels during arousal (Kalivendi et al., 2005). 
Immunoblotting analysis showed elevations of NFATc2 at late torpor in the skeletal muscle of ground squirrels. Although all NFATs are expressed in skeletal muscle and are important for proper muscle function, NFATc2 and c 3 are mostly commonly associated with myoblast fusion and muscle repair (Armand et al., 2008; Cho et al., 2007; Demonbreun et al., 2010; Horsley et al., 2001). Therefore, the increase in NFATc2 protein levels at ET (Figure 3.2) and the increase in NFATc3-DNA binding at LT (Figure 3.5) are indicative of increased skeletal muscle regeneration and repair in an effort to maintain skeletal muscle mass despite disuseinduced muscle wasting during torpor (Hindle et al., 2014). During LT, 2.08-fold and 2.37-fold rises in calcineurin and calpain1 levels respective, relative to EC were observed and this correlates with the rise in NFATc3 (Figure 3.4). Similar to NFATc3-DNA binding, myoferlin protein levels increased significantly during torpor as well; rising at EN, and peaking at ET and LT before decreasing to euthermic levels (Figure 3.4). Therefore, despite not showing significant differences in protein levels, the large increase in NFATc3 activity during torpor may be driving the increase in myoferlin levels that maintain skeletal muscle mass (Demonbreun et al., 2010). This increase in myoferlin supports the hypothesis that there is an increase in muscle regeneration to preserve skeletal muscle during torpor.

Having established the important role of $\mathrm{Ca}^{2+}$ in regulating the NFAT-calcineurin pathway and muscle maintenance during hibernation through $\mathrm{Ca}^{2+}$-binding proteins, we became interested in determining whether $\mathrm{Ca}^{2+}$ can directly affect NFAT binding to target promoters during torpor. Several studies have previously shown that intranuclear $\mathrm{Ca}^{2+}$ can regulate gene expression by directly binding to DNA or through regulation of transcription factors and their cofactors (Chawla, Hardingham, Quinn, \& Bading, 1998; Dobi \& Agoston, 1998; Pusl et al., 2002; Thompson et al., 2003). We identified using a modified environmental DPI-ELISA that $\mathrm{Ca}^{2+} \mathrm{did}$ 
indeed affect the binding of NFAT transcription factors to DNA during torpor. It was observed that progressively increasing $\left[\mathrm{Ca}^{2+}\right]$ decreased the binding of NFATc1, whereas NFATc4 showed increased binding to DNA when $\left[\mathrm{Ca}^{2+}\right]$ was increased to $600 \mathrm{nM}$ (Figure 3.7B). These effects of intranuclear $\mathrm{Ca}^{2+}$ on NFAT-DNA binding during torpor seem to be specific for each NFAT transcription factor, therefore this effect is likely not due to the binding and blocking of DNA by intranuclear $\mathrm{Ca}^{2+}$ (Dobi \& Agoston, 1998). This effect is most likely due to $\mathrm{Ca}^{2+}$ regulation of specific export kinases like calmodulin-dependent protein kinase IV (CAMKIV) or through specific coactivators of individual NFATs, such as CREB-binding protein (CBP) (Chawla et al., 1998; Yang, Davis, \& Chow, 2001). Given that urea is another key metabolite that is crucial during hibernation, specifically torpor, we created an environmental DPI-ELISA to test whether urea could affect NFAT binding activity as well (Epperson et al., 2011; Stenvinkel et al., 2013). Not surprisingly, urea did not have a significant effect on NFAT-binding to DNA in any of the tested conditions during LT (Figure 3.7A). Theoretically, the nuclear membrane allows compounds of $60 \mathrm{kDa}$ or less to pass through into the nucleus, and urea is just over that cut-off (Gerace \& Burke, 1988). Therefore, it would not be able to translocate into the nucleus.

Given the extreme variations in temperature that occurs during torpor-arousal cycles from euthermia $\left(37^{\circ} \mathrm{C}\right)$ to torpor $\left(0-4^{\circ} \mathrm{C}\right)$, we were interested in knowing whether temperature could potentiate or inhibit the binding of transcription factors, such as NFATs (Frerichs \& Hallenbeck, 1998; Storey \& Storey, 2004; Storey, 2010; Wang \& Lee, 2011). We carried out a modified DPIELISA, adjusting for the ambient temperature during the protein incubation step where binding between transcription factors and the DNA oligonucleotide occurs. We found that there were dramatic differences in transcription factor binding of NFATc1, c3, and c4 as the temperature was progressively decreased from euthermic $(\mathrm{EC}) \mathrm{T}_{\mathrm{b}}\left(37^{\circ} \mathrm{C}\right)$ to the depressed $\mathrm{T}_{\mathrm{b}}$ seen during LT 
$\left(4^{\circ} \mathrm{C}\right)$ (Figure 3.6A-C). When comparing the declines in NFAT-DNA binding from EC to LT, we can see that there was a lesser decrease in the binding activity of NFATc3 $(64 \%)$ relative to NFATc1 (89\%) and NFATc4 (93\%) (Figure 3.6C). This relatively smaller decrease in NFATc3 binding activity could partly explain the preservation of skeletal muscle mass during torpor, as NFATc3 plays the most vital role in coordinating muscle remodeling out of the four NFATs (Armand et al., 2008; Delling et al., 2000; Demonbreun et al., 2010; Hudson et al., 2014). Due to metabolic rate depression and the need to conserve ATP during torpor, the expression of nonessential genes is likely halted, therefore NFATc1 and c4 activity show a greater decline compared to NFATc3. This study is the first to identify changes in transcription factor-DNA binding affinity that are temperature-dependent, although further studies need to be conducted to determine whether our findings are specific for NFAT transcription factors or if it reflects a greater number of transcription factors. More importantly, further studies need to determine whether the temperature-sensitivity of NFAT transcription factors are due to conformational changes that occur at lower temperatures to the protein itself, to DNA, or if it has to do with interactions with temperature-sensitive cofactors. For example, NFATc2 has been shown to cooperate with heat shock transcription factor 1 (HSF1), which is responsible for regulating the gene expression of other heat shock proteins (Hayashida et al., 2010).

In summary, the present study provides insight into some of the key proteins involved in skeletal and cardiac muscle remodeling during hibernation. Although more remains to be investigated, including the regulation of factors associated with the UPS (Chapter 5), the results from this study indicate that NFATc1-4 as well as the targets myoferlin and myomaker are important to the hypertrophy and preservation of skeletal muscle mass and function during hibernation. Our findings also demonstrate that $\mathrm{Ca}^{2+}$ signaling plays a key role in regulating the 
NFAT-calcineurin pathway in skeletal muscle over the torpor-arousal cycle. In addition, in this chapter, a novel technique, the environmental DPI-ELISA, was developed and used to study the effects of environmental stimuli such as temperature, [urea], and $\left[\mathrm{Ca}^{2+}\right]$ on transcription factor binding to target promoters, which was the secondary objective of this study. We found that [urea] has little effect on NFAT binding, but intranuclear $\left[\mathrm{Ca}^{2+}\right]$ seems to affect the DNAbinding activity of both NFATs $\mathrm{c} 1$ and $\mathrm{c} 4$, possibly through $\mathrm{Ca}^{2+}$ regulation of export kinases and coactivators. Furthermore, we determined that temperature differences from euthermia $\left(37^{\circ} \mathrm{C}\right)$ to torpor $\left(4^{\circ} \mathrm{C}\right)$ have profound effects on the binding of NFATc1, $\mathrm{c} 3$, and $\mathrm{c} 4$ although the effects are more pronounced for NFATs $\mathrm{c} 1$ and $\mathrm{c} 4$. The novel finding that TF-binding to DNA is temperature dependent should be explored further for other TFs and to identify potential mechanisms. These findings contribute to our understanding of muscle remodeling, and these mechanisms involved in preserving ground squirrel skeletal muscle throughout the torpor-arousal cycle make studying the ground squirrel biologically-relevant. Promising therapeutics for muscle wasting include anabolic androgens, such as nandrolone, which has been shown to activate calcineurin-NFAT signaling and reduced denervation-induced muscle atrophy in mice (Qin, Pan, Wu, Bauman, \& Cardozo, 2015). 


\section{Figures}

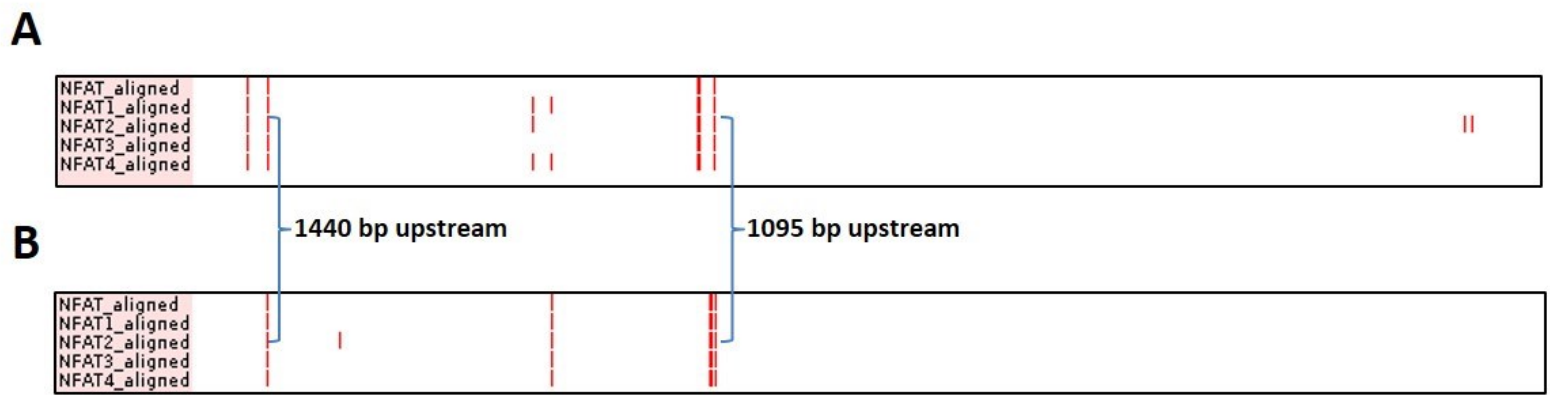

C GGGAAGGAAAIGTGCGGGTGGTCAC

Figure 3.1: DNA sequence analysis of the myomaker promoter in multiple animals to find putative NFAT binding sites. The sequence $1500 \mathrm{bp}$ upstream of the (a) squirrel and mouse, and (b) squirrel and human myomaker transcriptional start sites were interrogated using rVista for the detection of NFATc1-4 binding sequences. Red lines indicate aligned hits of possible NFATc1-4 binding regions between organisms, the two sequences highlighted were identified by rVista as possible binding sites. Cross-referencing with NFAT binding sequence from literature (c, shown in green), one probable binding site remained 1095 bp upstream of the myomaker transcriptional start site, this sequences along with its aligned flanking region from the multiple alignments was used to design a 25 bp probe for DNA ELISA. Figure from (Zhang \& Storey, 2015). 


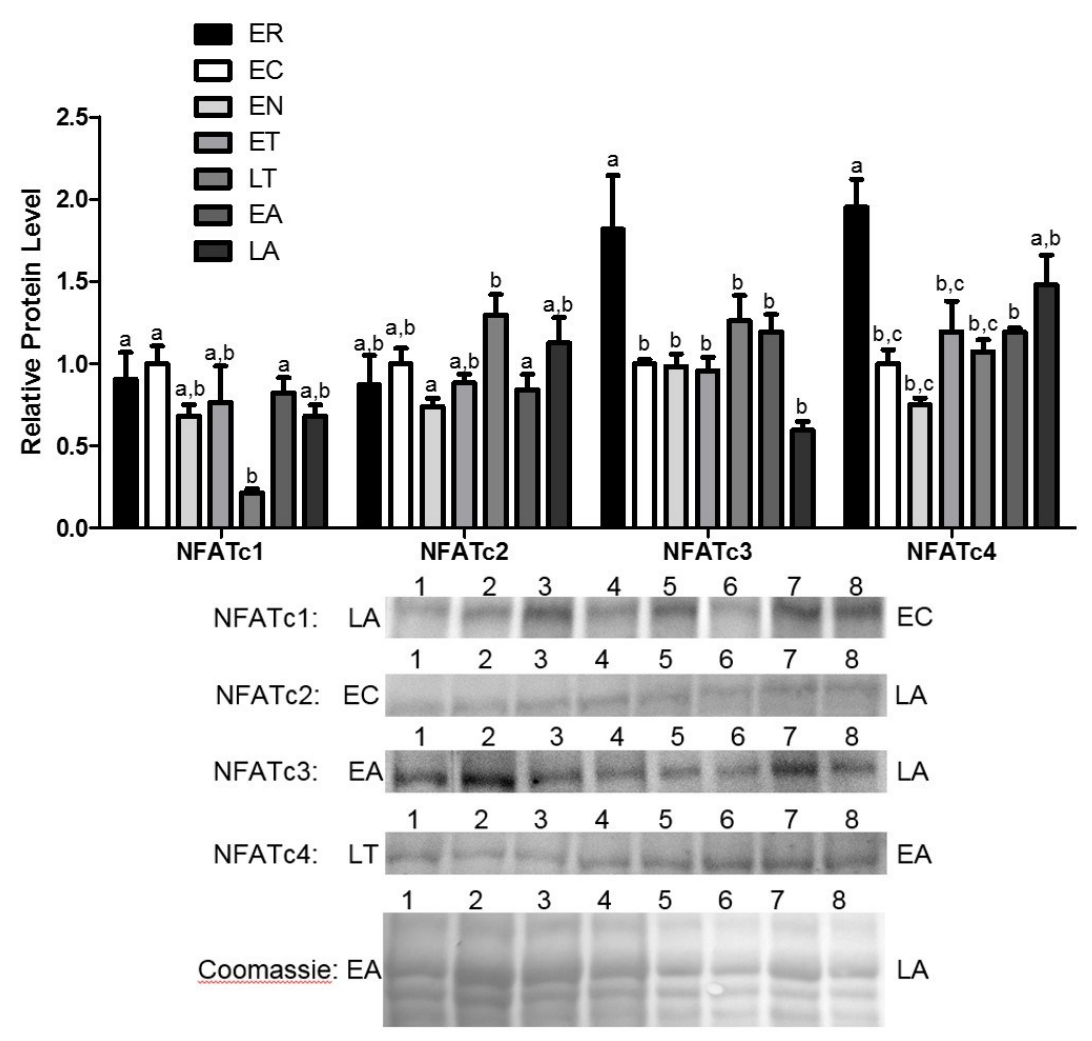

Figure 3.2: Changes in the protein levels of NFAT transcription factors over the course of the torpor-arousal cycle in skeletal muscle of I. tridecemlineatus. NFATc1, c2, c3, and c4 total protein expression levels were visualized at seven sampling points: ER, EC, EN, ET, LT, EA, LA. See Materials and methods for more extensive definitions. Representative Western blots and Coomassie total protein loading controls are shown for selected pairs of sampling points that are labeled to the left and right of the gel. Sample numbers (lanes) are labeled along the top indicating 4 samples of one type (e.g., EC lanes 1, 2, 3, 4) and 4 samples of another (e.g. LA lanes $5,6,7,8)$. Also shown are histograms with mean standardized band densities ( \pm S.E.M., $\mathrm{n}=4$ independent protein isolations from different animals). Data was analyzed using a one-way analysis of variance with a post hoc Tukey's test $(\mathrm{p}<0.05)$; for each parameter measured, values that are not statistically different from each other share the same letter notation. Figure from (Zhang \& Storey, 2015). 


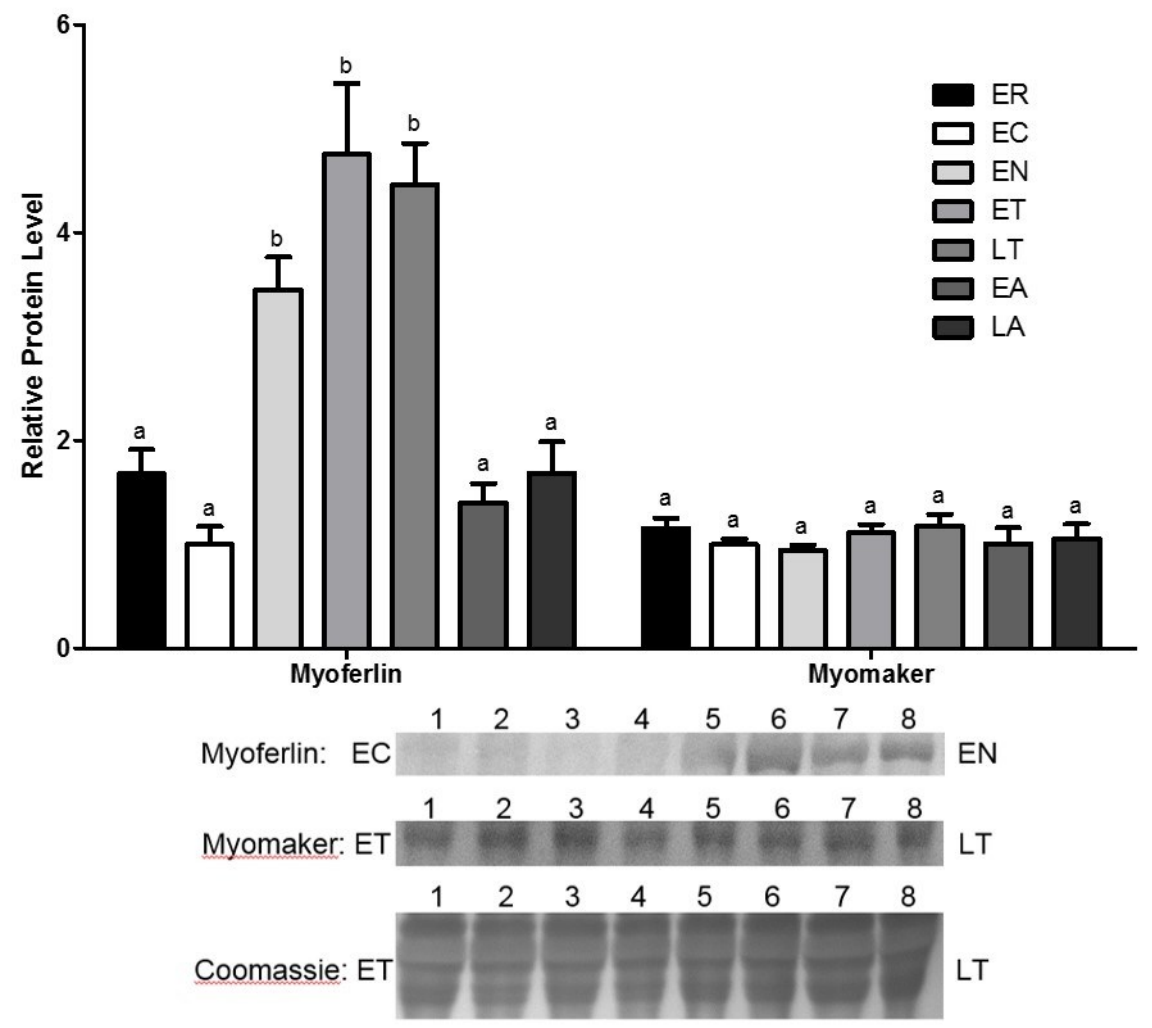

Figure 3.3: Changes in myoferlin and myomaker total protein levels in skeletal muscle over the torpor-arousal cycle in I. tridecemlineatus. Myoferlin and Myomaker total protein expression levels were visualized at seven sampling points: ER, EC, EN, ET, LT, EA, LA. Representative Western blots and Coomassie total protein loading controls are shown for selected pairs of sampling points that are labeled to the left and right of the gel. Sample numbers (lanes) are labeled along the top indicating 4 samples of one type (e.g., EC lanes 1, 2, 3, 4) and 4 samples of another (e.g. EN lanes 5, 6, 7, 8) using Myoferlin as an example. Also shown are histograms with mean standardized band densities ( \pm S.E.M., $n=4$ independent protein isolations from different animals). Data was analyzed using a one-way analysis of variance with a post hoc Tukey's test $(\mathrm{p}<0.05)$; for each parameter measured, values that are not statistically different from each other share the same letter notation. Figure from (Zhang \& Storey, 2015). 


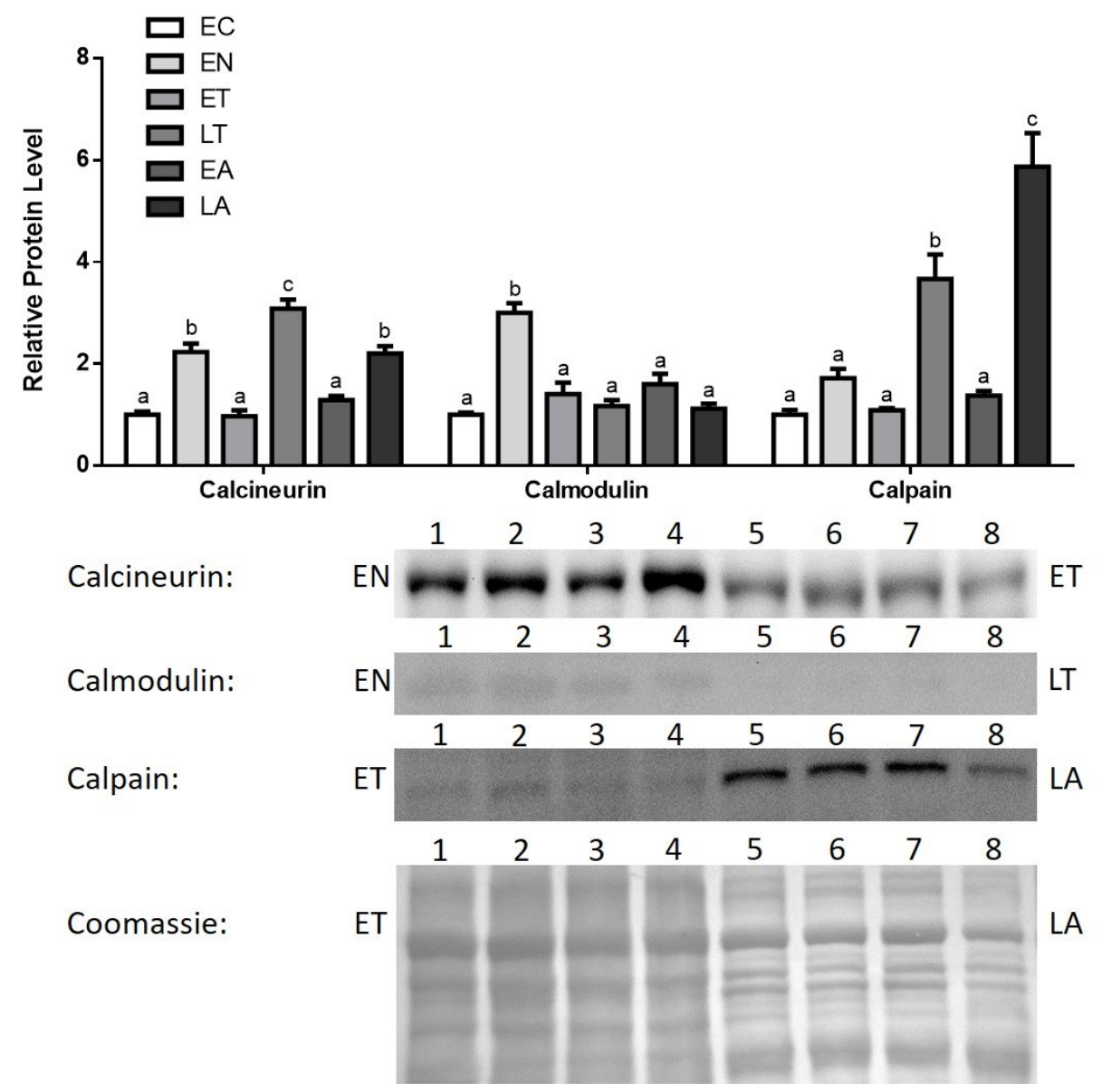

Figure 3.4: Changes in calcineurin, calmodulin, and calpain1 total protein levels in skeletal muscle over the torpor-arousal cycle in I. tridecemlineatus. Calcineurin, calmodulin, and calpain1 total protein expression levels were visualized at six sampling points: EC, EN, ET, LT, EA, LA. Representative Western blots and Coomassie total protein loading controls are shown for selected pairs of sampling points that are labeled to the left and right of the gel. Sample numbers (lanes) are labeled along the top indicating 4 samples of one type (e.g., EN lanes 1, 2, 3, 4) and 4 samples of another (e.g. ET lanes 5, 6, 7, 8) using Calcineurin as an example. Also shown are histograms with mean standardized band densities ( \pm S.E.M., $n=4$ independent protein isolations from different animals). Data was analyzed using a one-way analysis of variance with a post hoc Tukey's test $(\mathrm{p}<0.05)$; for each parameter measured, values that are not statistically different from each other share the same letter notation. 


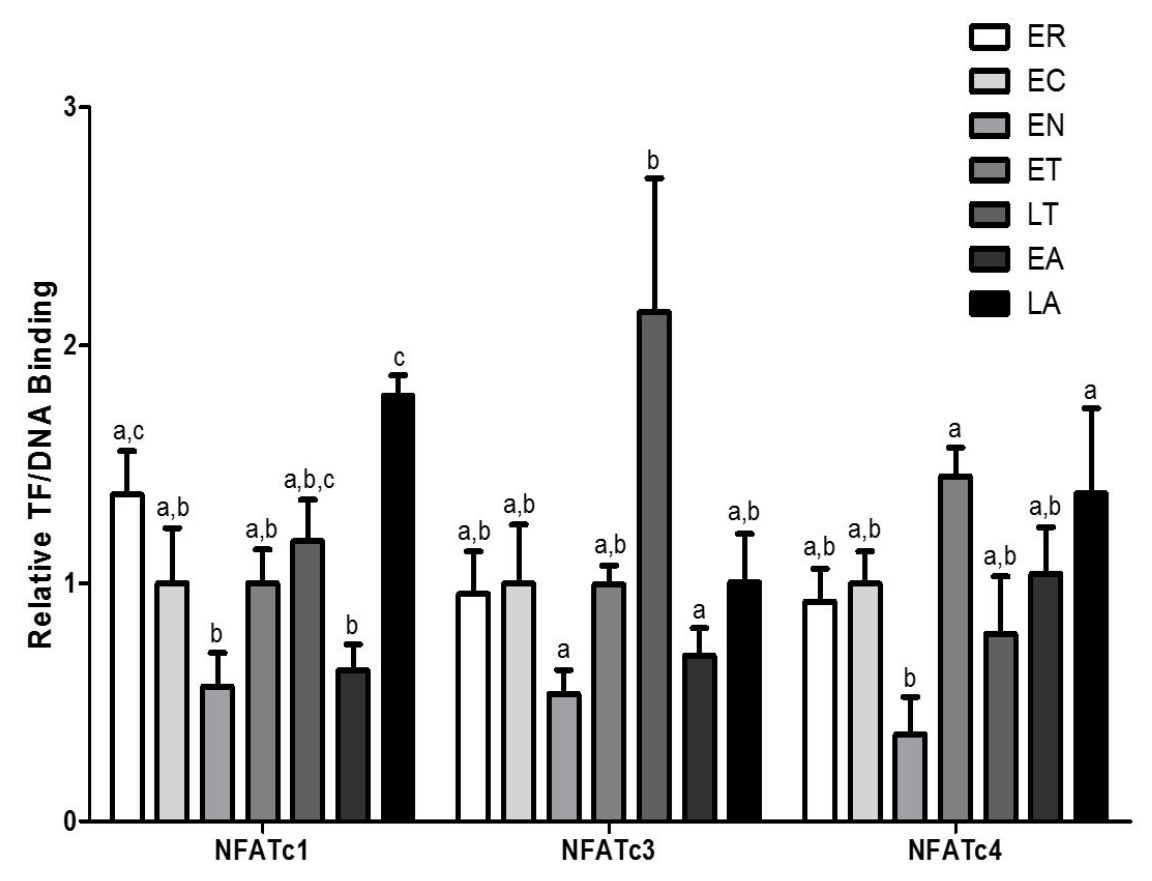

Figure 3.5: Changes in binding of the transcription factor NFATc1, NFATc3, and NFATc4 to a DNA-binding element designed for the NFAT consensus sequence in the skeletal muscle of $I$. tridecemlineatus over the torpor-arousal cycle. DNA-Protein Interaction (DPI)-ELISA absorbance readings were corrected by subtraction of negative controls containing no protein and values were expressed relative to EC. Histograms show mean relative values \pm S.E.M., $n=4$ independent biological replicates for each of the seven experimental conditions (ER, EC, EN, ET, LT, EA, LA). Refer to Figure 3.2 and the Materials and Methods section for more information about the seven sampling points. 


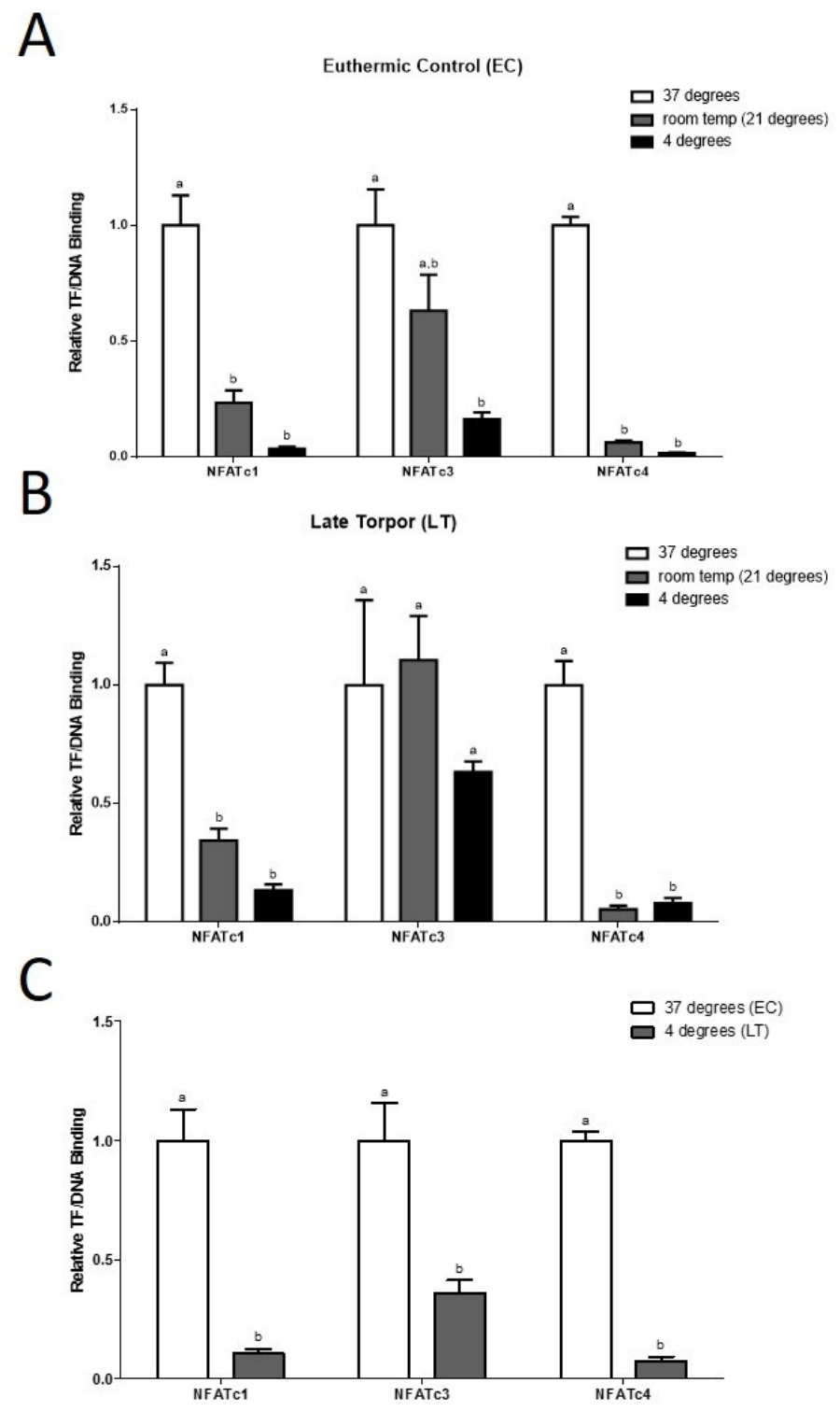

Figure 3.6: Effect of adjusting temperature on transcription factor-DNA binding of NFATc1, c3, and c4. (a) NFAT-DNA binding was measured at 37, 24 (room temperature), and $4^{\circ} \mathrm{C}$ at the $\mathrm{EC}$ sampling point before hibernation is initiated. (b) NFAT-DNA binding was measured at 37, 24 (room temperature), and $4^{\circ} \mathrm{C}$ at the LT sampling point deep within hibernation. (c) Changes in the binding of NFAT c1, c3, and c4 transcription factors to DNA at physiological temperatures from EC $\left(37^{\circ} \mathrm{C}\right)$ and LT $\left(4^{\circ} \mathrm{C}\right)$. Modified DPI-ELISA absorbance readings were corrected by subtraction of no protein controls, and values were expressed relative to $37^{\circ} \mathrm{C}$. 
A

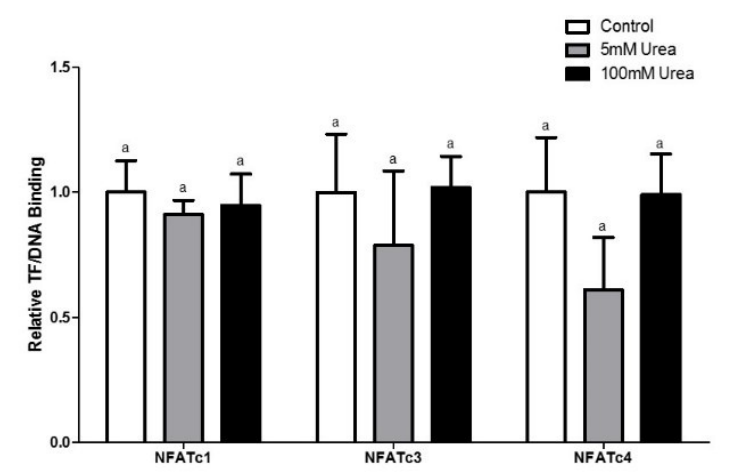

B

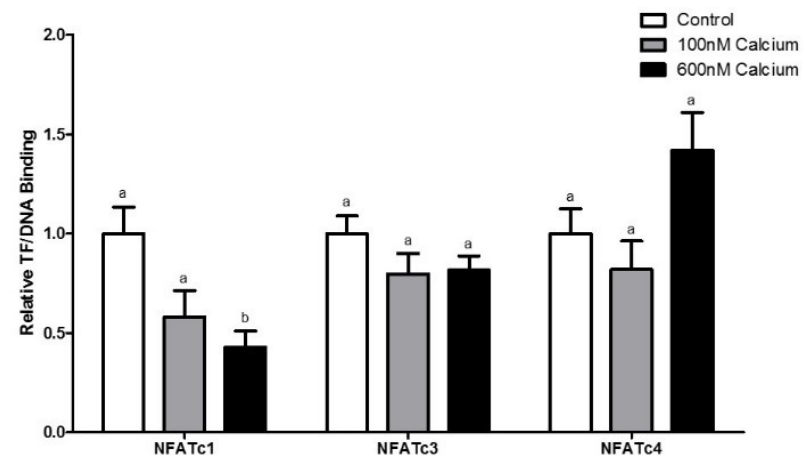

Figure 3.7: Effect of adding free urea and $\mathrm{Ca}^{2+}$ on transcription factor-DNA binding of NFATc1, c3, and c4. (a) Transcription factor-DNA binding was measured during the LT sampling point with no urea added (control), 5mM Urea added, and 100mM Urea added. (b) Transcription factor-DNA binding was measured during the LT sampling point with no $\mathrm{Ca}^{2+}$ added, $100 \mathrm{nM} \mathrm{Ca}^{2+}$ added, and $600 \mathrm{nM} \mathrm{Ca}^{2+}$ added. Modified DPI-ELISA absorbance readings were corrected by subtraction of negative control containing no protein, and values were expressed relative to the control (no $\mathrm{Ca}^{2+}$ or no urea added). 


\section{Chapter 4}

Nuclear factor of activated T cells regulates cardiac hypertrophy through calcium signaling during hibernation 


\section{Introduction}

As discussed in Chapters 1 and 3, each organ/tissue of the I. tridecemlineatus must make specific adjustments that allow them to maintain or readjust physiological function at low $\mathrm{T}_{\mathrm{b}}$ in order to survive torpor, and hibernation in general. Another tissue that undergoes physiological adaptations is the heart. As discussed in chapter 1, heart rate is reduced strongly during torpor, often to just $5-10$ beats/min in comparison with the euthermic rates of $350-400$ beats $/ \mathrm{min}$; this in addition to the increased viscosity of blood at low $\mathrm{T}_{\mathrm{b}}$ values required changes in cardiac dynamics (Frerichs \& Hallenbeck, 1998; Frerichs et al., 1994). As a result, the strength of each individual contraction must be significantly greater and as a result cardiomyocyte hypertrophy is observed (Nakipova et al., 2007; Storey \& Storey, 2004; Wickler et al., 1991). This adaptation is accompanied by enhanced protein synthesis, adjustments to the structure of sarcomeres, as well as changes in gene transcription (Frey, Katus, Olson, \& Hill, 2004; Li et al., 2013; Nelson \& Rourke, 2013; Tessier \& Storey, 2012). Interestingly, hibernators are able to undergo reversible cardiac hypertrophy, where the heart hypertrophies during hibernation but this process is reversed while coming out of hibernation, and this may be related to molecular adaptations that are just beginning to be discovered (Fahlman et al., 2000; Nelson \& Rourke, 2013; Tessier \& Storey, 2012; Yan, Kudej, Vatner, \& Vatner, 2015). Understanding how these animals can rapidly and effectively reverse cardiac hypertrophy would provide novel insight into understanding and perhaps treating cardiomyopathies and heart failure.

The present chapter focuses again on the role of calcium signaling in regulating the NFAT family of transcription factors and their downstream targets, which are suspected to play a crucial role in cardiac muscle remodeling. NFATs may contribute to the adaptive responses by all muscle cell types in the body, including those needed to remodel cardiac muscles during 
hibernation. Cardiac expression of NFAT has been shown to regulate cardiomyocyte atrophy, apoptosis, development, and growth (Li et al., 2013; Lin et al., 2009; Liu et al., 2006; Molkentin et al., 1998; Schubert et al., 2003). With regard to hibernation, the use of isobaric tag for relative and absolute quantification (iTRAQ) technology with cardiac tissue of hibernating woodchucks (Marmota monax) demonstrated an up-regulation of the NFAT pathway during hibernation. Furthermore, expression of constitutively-active calcineurin or NFATc4 in cardiac tissue of transgenic mice induced cardiac hypertrophy (Molkentin et al., 1998). As discussed in Chapter 3, NFATs are activated via dephosphorylation by calcineurin, a calmodulin-stimulated protein phosphatase, allowing NFATs to translocate to the nucleus and bind to transcriptional complexes of their target genes (Rusnak \& Mertz, 2000). Calmodulin (CAM) is a calcium-binding protein that controls various signaling pathways, including the NFAT-calcineurin pathway. Another important protein that regulates the NFAT-calcineurin pathway is the calcium-dependent protease calpain. $\mu$-calpain (calpain1) causes proteolysis of the auto-inhibitory domain of calcineurin, which causes it to translocate to the nucleus and become constitutively active (Burkard, 2005; Lee et al., 2014; Shioda et al., 2006; Wu et al., 2004). Therefore, calpain and CAM both play vital roles in regulation of the NFAT-calcineurin pathway and NFATs may contribute to the adaptive responses needed for cardiac muscle remodeling during hibernation (Figure 1.3).

Considering the unique tendencies of hibernators to undergo reversible cardiac hypertrophy by remodeling their cardiac muscle over the course of the hibernation season, we hypothesized that $\mathrm{Ca}^{2+}$ signaling regulation of the NFAT-calcineurin pathway would play a significant role in this process, particularly by modulating the gene expression of selected proteins fundamental to muscle design. As discussed in chapter 3, myoferlin is one such protein 
that is highly expressed in skeletal muscle and to a lesser degree in cardiac muscle (Davis et al., 2000). It contains multiple NFAT-binding sites in its promoter, which drives high levels of myoferlin expression in vivo and in vitro, allowing it to act as a muscle membrane protein that promotes hypertrophy (Davis et al., 2002; Doherty et al., 2005). Similarly, myomaker is another a membrane protein found in muscle that appears to play an essential role in muscle development, and we demonstrated a novel finding in the previous chapter that the promoter of myomaker contains NFAT-binding domains as well (Millay et al., 2013). The present chapter analyzed the protein expression of calcineurin, calmodulin, calpain, NFATc1-4, myoferlin, and myomaker over the torpor-arousal cycle in the cardiac muscle of thirteen-lined ground squirrels.

\section{Materials and Methods}

Animals

All animals were captured, treated, and their tissues were harvested following the same protocol as previously described in Chapter 2. The cardiac tissue used was a mixture of atrial and ventricular tissue.

\section{Total Protein Extract Preparations}

Samples of frozen cardiac muscle (approximately $500 \mathrm{mg}$ ) were homogenized for total protein extraction as previously described. Total protein extracts were used for western blotting experiments as previously described in Chapter 2.

\section{Western Blotting}

Western blotting was performed as described in Chapter 2 and 3 for six time points (EC, EN, ET, LT, EA, IA) for NFATc1-4, myoferlin, and myomaker, calcineurin, CAM, and 
calpain1. Equal amounts of protein from each sample $(25 \mu \mathrm{g})$ were loaded onto $6 \%$ (NFATc1-4, myoferlin), $8 \%$ (calcineurin, calpain 1), or 15\% (myomaker, CAM) polyacrylamide gels and were run at $180 \mathrm{~V}$ for $60-120 \mathrm{~min}$. For calmodulin, $35 \mu \mathrm{g}$ of protein for each sample were loaded on the polyacrylamide gels. Proteins were then transferred to PVDF membranes by electroblotting at $320 \mathrm{~mA}$ for $60 \mathrm{~min}$ (myomaker), $90 \mathrm{~min}$ (calcineurin, calpain1), $120 \mathrm{~min}$ (NFATc1-3, myoferlin), or at $30 \mathrm{~V}$ for $100 \mathrm{~min}$ (calmodulin). Membranes incubated in primary antibodies were blocked for 30 min with 2.5 (for NFATc1-4) or 5\% (for CnA, CAM, calpain1, myoferlin and myomaker) w:v milk in 1x TBST.

Antibodies specific for mammalian NFATc1-4, myoferlin, and myomaker, calcineurin, CAM, and calpain1 were provided by the manufacturers indicated in Chapter 3. NFATc1-4, myoferlin, and myomaker antibodies were used at a 1:500 v:v dilution in 1x TBST. All other antibodies were used at a 1:1000 v:v dilution in 1x TBST. All primary antibody incubations took place over one night. Membranes that had been probed with myomaker (TMEM8c) were incubated with HRP-linked anti-goat IgG secondary antibody (BioShop: 1:6000 v:v dilution). All other antibodies were detected using HRP-linked anti-rabbit IgG secondary antibody (Bioshop: 1:6000 v:v dilution). All secondary antibody incubations were 30 minutes. The primary antibodies cross-reacted with a single band on immunoblots at molecular weights indicated in Chapter 3. Other parts of the procedure were carried out in accordance with the protocol described in Chapter 2.

\section{Quantification and Statistics}

Band densities for NFATc1-4, myoferlin, myomaker, calcineurin, CAM, and calpain-1 on chemiluminescent immunoblots were visualized and quantified as described in Chapter 2. Data are expressed as means \pm SEM, $\mathrm{n}=4$ independent samples. 


\section{Results}

Analysis of NFATc1-4 protein levels in cardiac muscle

Analysis was performed on NFAT c1-4 protein levels by comparing immunoblots of cardiac muscle from six sampling points on the torpor-arousal cycle: EC, EN, ET, LT, EA, IA (Figure 4.1). In cardiac tissue, NFATc1 levels did not change significantly over the course of the torpor-arousal time course. On the other hand, NFATc2 levels increased during torpor, peaking at ET (1.88-fold increase from EN, $\mathrm{p}<0.05)$. NFATc3 protein levels were unchanged over the torpor-arousal cycle. Lastly, NFATc4 levels remained stable over torpor, but increased during arousal, peaking at IA (1.66 fold higher than ET, $\mathrm{p}<0.05)$ (Figure 4.1).

Analysis of myoferlin and myomaker (TMEM8c) protein levels

Similar to skeletal muscle in Chapter 3 (Figure 3.3), myoferlin levels in cardiac muscle rose dramatically at ET (2.68 fold higher in comparison with control, $\mathrm{p}<0.05)$. In contrast to skeletal muscle levels, myomaker in cardiac muscle also peaked at ET (1.88 fold higher in comparison with control, $\mathrm{p}<0.05$ ) (Figure 4.2).

Analysis of calcineurin A, calmodulin, and calpain1 protein levels in cardiac muscle

CnA protein levels remained fairly stable throughout the torpor-arousal cycle, with the exception of IA, where levels decreased dramatically by $62 \%$ from EA $(p<0.05)$. Calmodulin levels were highest at EC and EN, but levels began to decrease dramatically upon entering arousal, with the lowest levels being observed at EA ( $70 \%$ decrease in comparison with EC, $\mathrm{p}<0.05$ ). Calpain levels showed a similar trend, with the highest levels being observed at EC and IA, and drastically lower levels throughout the torpor-arousal cycle. The lowest levels were observed during EN ( $85 \%$ decrease from EC, $\mathrm{p}<0.05)$ (Figure 4.3). 


\section{Discussion}

The current chapter aimed at furthering our understanding of the molecular mechanisms underlying cardiac muscle remodeling during hibernation in the 13-lined ground squirrel. As discussed in chapter 1 , I. tridecemlineatus is an excellent animal model for studying cardiac muscle remodeling with applications for the treatment of cardiac hypertrophy and heart failure because its cardiac muscle is known to naturally undergo reversible cardiac hypertrophy (Li et al., 2013; Nelson \& Rourke, 2013). However, the molecular mechanisms that are responsible the changes of muscle structure/function during hibernation are not well known. Therefore, the present study focuses on the family of transcription factors known as NFATs, which have been shown to regulate targets associated with cardiomyocyte hypertrophy, apoptosis, and development (Li et al., 2013; Lin et al., 2009; Liu et al., 2006; Molkentin et al., 1998; Tessier \& Storey, 2012).

NFATs are regulated by calcineurin, a calmodulin-stimulated phosphatase that dephosphorylates NFATs, thus allowing them to translocate into the nucleus (Rusnak \& Mertz, 2000). Calcineurin is sensitive to changes in calcium levels and it is positively regulated by other calcium-signaling proteins such as CAM and calpain (Al-Shanti \& Stewart, 2009; Burkard, 2005; Lee et al., 2014; Shibasaki et al., 2002; Shioda et al., 2006; Wu et al., 2004; Yang \& Klee, 2000). Therefore, experiments were conducted to study the roles of calcineurin, CAM, and calpain as upstream regulators of the NFAT pathway within the context of reversible cardiac hypertrophy during hibernation. Furthermore, the downstream targets myoferlin and myomaker were evaluated because of their newly identified and crucial role as muscle membrane proteins in muscle development and repair (Demonbreun et al., 2010; Doherty et al., 2005; Millay et al., 2013, 2014). 
In cardiac muscle, the response of NFATc1-4 transcription factors as well as the downstream targets myoferlin and myomaker were enhanced during torpor (Figures 4.1, 4.2). Similarly to skeletal muscle, NFATc2 was significantly elevated during torpor. Although myoferlin was elevated in both heart and skeletal muscle, myomaker remained stable in skeletal muscle throughout hibernation. In the heart, myomaker showed a spike at ET (1.88 fold higher in comparison with control, $\mathrm{p}<0.05$ ) (Figure 4.2). The regulator of NFATs, calcineurin decreased during arousal by $62 \%$ from EA to IA ( $\mathrm{p}<0.05)$. One of the activators of calcineurin, CAM, also decreased during LT and throughout arousal. Similarly, calpain levels declined from EC to EA by $61 \%(\mathrm{p}<0.05)$. Calpain cleaves the autoinhibitory domain of calcineurin, thus activating it (Burkard, 2005; Lee et al., 2014; Shioda et al., 2006; Wu et al., 2004). Therefore, these $\mathrm{Ca}^{2+}$ signaling proteins (CAM, and calpain) as well as the NFAT-CnA pathway and its downstream muscle targets (myoferlin and myomaker) could be part of the mechanism regulates reversible cardiac muscle hypertrophy. During torpor, NFATc2 could be causing upregulation of myoferlin, thus promoting cardiac hypertrophy to increase contractility to the heart in response to the decrease in heart rate during hibernation (Frerichs \& Hallenbeck, 1998; Frerichs et al., 1994). Then, the mechanism behind the rapid reduction of cardiac mass may be initiated by decreases in calcineurin activity, which is the result of lower calpain and CAM levels upon and before entering arousal. Subsequently, NFATc2 levels decrease and this may be causing the downregulation of both myoferlin and myomaker, thus reversing the cardiac hypertrophic stimulus. Although the NFAT-calcineurin pathway has been studied in association with cardiac hypertrophy in the past, the present findings identify a novel mechanism of rapidly naturally reversing cardiac hypertrophy, whereby decreased calcium signaling results in a downregulation 
of muscle hypertrophy proteins targeted by NFATs (Li et al., 2013; Lin et al., 2009; Molkentin et al., 1998).

In summary, the present chapter provides insight into some of the key proteins involved in cardiac muscle remodeling during hibernation. Although more remains to be investigated, including the regulation of factors associated with muscle atrophy and the reduction in cardiac dimensions (Chapter 6), the results from this chapter indicate that the $\mathrm{Ca}^{2+}$ signaling proteins (calcineurin, calmodulin, calpain) regulating the activity of NFATc1-4. as well as their downstream targets, myoferlin and myomaker, are important to the hypertrophy of cardiac muscle during hibernation. This is also the first study that has found the myomaker protein to be expressed in the heart, although its specific role still remains unclear. Further elucidation of the role that the Calcineurin-NFAT pathway plays in reversible cardiac hypertrophy in thirteen-lined ground squirrels will not only contribute to our understanding of cardiac remodeling, it could potentially lead to the applications for treating maladaptive cardiac hypertrophy and heart failure. Specifically, the identification of calpain and calmodulin as initiators for the reversal of cardiac hypertrophy in squirrels shows promise. The unique and tissue-specific mechanisms in ground squirrel of preserving skeletal muscle (Chapter 3) and undergoing reversible cardiac hypertrophy during the torpor-arousal cycle make studying this animal biologically and clinically-relevant. 


\section{Figures}

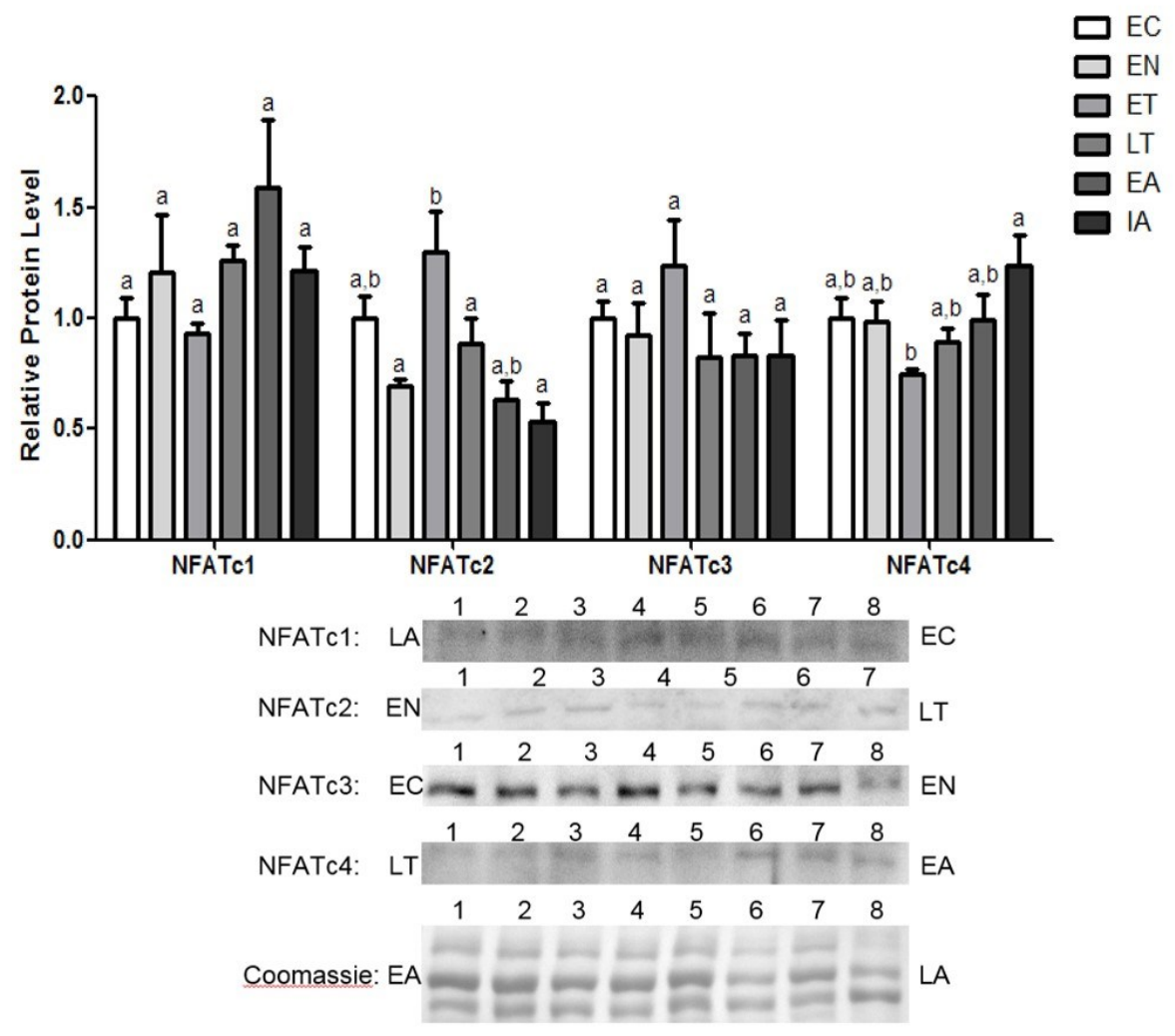

Figure 4.1: Changes in the protein levels of NFAT transcription factors over the course of the torpor-arousal cycle in cardiac muscle of I. tridecemlineatus. NFATc1, c2, c3, and c4 total protein expression levels were visualized at six sampling points: EC, EN, ET, LT, EA, and IA. Representative Western blots and Coomassie total protein loading controls are shown for selected pairs of sampling points that are labeled to the left and right of the gel. Sample numbers (lanes) are labeled along the top indicating 4 samples of one type (e.g., EN lanes 1, 2, 3, 4) and 4 samples of another (e.g. LT lanes 5, 6, 7, 8) using NFATc2 as an example. Also shown are histograms with mean standardized band densities ( \pm S.E.M., $n=4$ independent protein isolations from different animals). Data was analyzed using a one-way analysis of variance with a post hoc Tukey's test $(\mathrm{p}<0.05)$; for each parameter measured, values that are not statistically different from each other share the same letter notation. Figure from (Zhang \& Storey, 2015). 


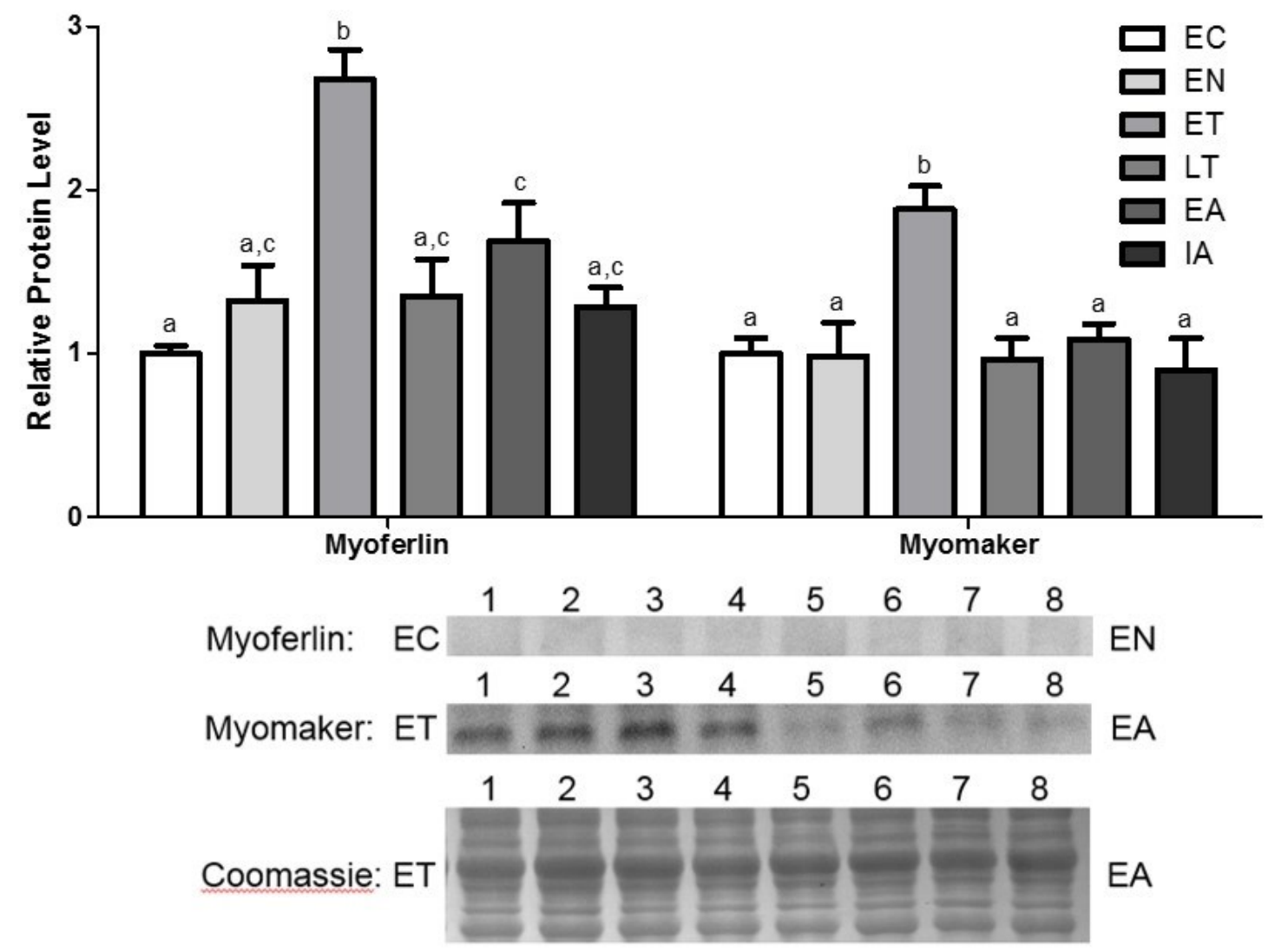

Figure 4.2: Changes in myoferlin and myomaker total protein levels in cardiac muscle over the torpor-arousal cycle in I. tridecemlineatus. Representative Western blots and Coomassie total protein loading controls are shown for selected pairs of sampling points that are labeled to the left and right of the gel. Sample numbers (lanes) are labeled along the top indicating 4 samples of one type (e.g., EC lanes 1, 2, 3, 4) and 4 samples of another (e.g. EN lanes 5, 6, 7, 8) using Myoferlin as an example. Also shown are histograms with mean standardized band densities $( \pm$ S.E.M., $\mathrm{n}=4$ independent protein isolations from different animals). Data was analyzed using a one-way analysis of variance with a post hoc Tukey's test $(\mathrm{p}<0.05)$; for each parameter measured, values that are not statistically different from each other share the same letter notation. Figure from (Zhang \& Storey, 2015). 


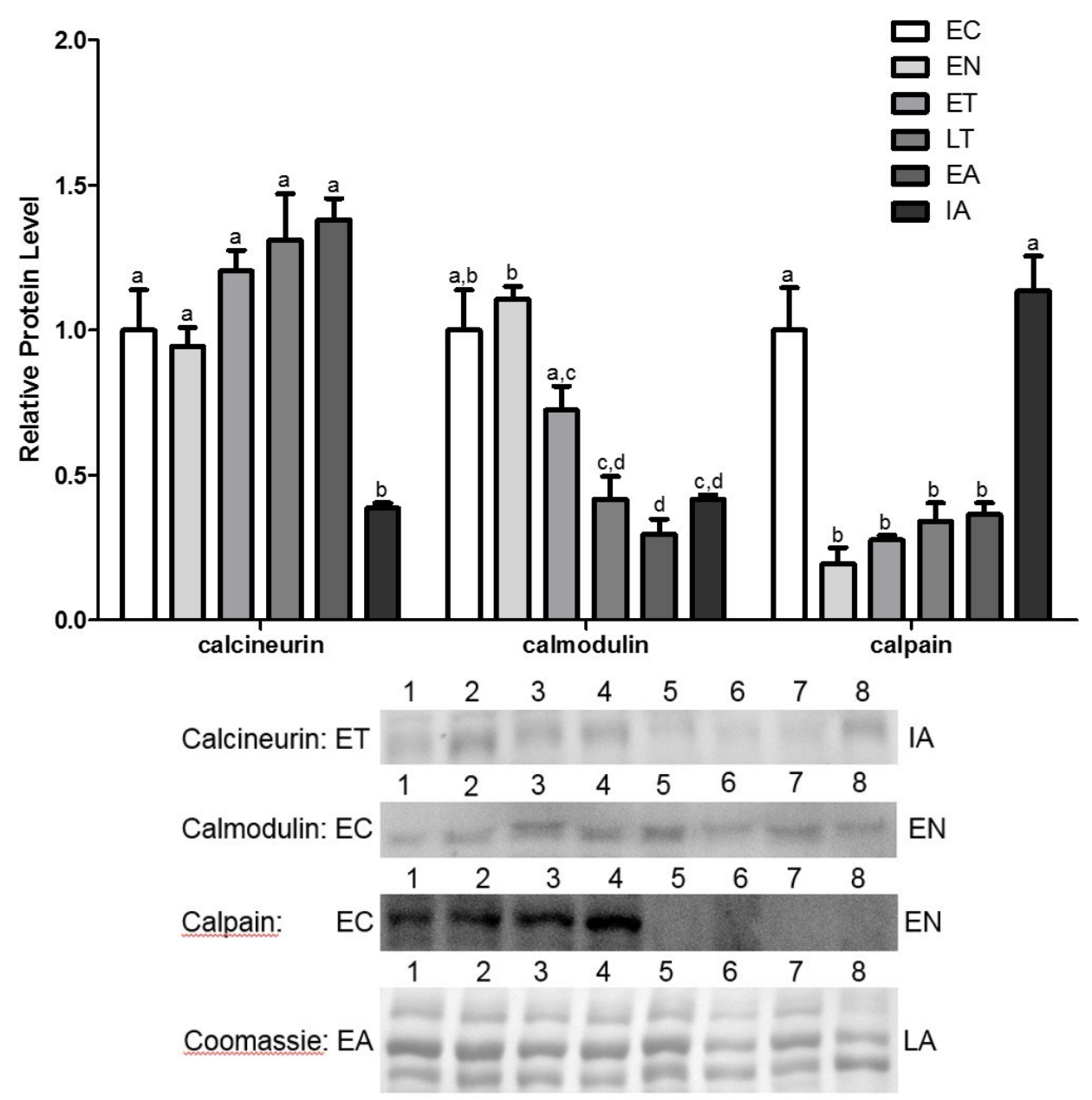

Figure 4.3: Changes in calcineurin, calmodulin, and calpain total protein levels in cardiac muscle over the torpor-arousal cycle in I. tridecemlineatus. Representative Western blots and Coomassie total protein loading controls are shown for selected pairs of sampling points that are labeled to the left and right of the gel. Sample numbers (lanes) are labeled along the top indicating 4 samples of one type (e.g., EC lanes 1, 2, 3, 4) and 4 samples of another (e.g. EN lanes 5, 6, 7, 8) using Calmodulin as an example. Also shown are histograms with mean standardized band densities ( \pm S.E.M., $n=4$ independent protein isolations from different animals). Data was analyzed using a one-way analysis of variance with a post hoc Tukey's test $(\mathrm{p}<0.05)$; for each parameter measured, values that are not statistically different from each other share the same letter notation. Figure from (Zhang \& Storey, 2015). 


\section{Chapter 5}

Regulation of Foxo4 and MyoG promotes skeletal muscle atrophy during torpor in ground squirrels 


\section{Introduction}

As discussed in Chapter 1, I. tridecemlineatus needs to make significant adaptations in order to survive the periods of low $\mathrm{T}_{\mathrm{b}}$ during torpor, the fluctuations in $\mathrm{T}_{\mathrm{b}}$ as a result of the torpor-arousal cycles, and for bodily functions to be restored following hibernation so that it can resume natural activities and scavenge for food. In order to resume post-hibernation activities, hibernators need to avoid significant disuse-induced skeletal muscle atrophy (muscle protein degradation) during hibernation, otherwise muscle mass, strength, and relative amount of oxidative muscle fibers will all be reduced (Bassel-Duby \& Olson, 2006; Choi et al., 2009; Malatesta et al., 2009; Rourke et al., 2004). Interestingly, it was demonstrated that the relative ratio of muscle mass/body weight actually increases throughout hibernation in ground squirrels due to significant losses in body weight as well as an extremely effective mechanism of muscle preservation and remodeling that is unique to hibernators (Gao et al., 2012). Therefore, uncovering the molecular mechanisms underlying this process of muscle maintenance, that occurs naturally, have clinical relevance for therapeutic intervention of muscle wasting and assisting in the physical rehabilitation.

Recent studies have begun to elucidate the molecular basis of muscle remodeling and preservation, with findings indicating that the peroxisome proliferator-activated receptor $\gamma$ coactivator 1- $\alpha$ (PGC-1 $\alpha)$ and the NFAT family of transcription factors are implicated in this process (Xu et al., 2013; Zhang \& Storey, 2015). However, a current gap in knowledge exists around whether the molecular pathways of muscle atrophy and protein degradation are still activated during hibernation as a result of inactivity. It is important to understand whether muscle remodeling and hypertrophy pathways involving PGC-1 $\alpha$ and NFAT simply balance the 
muscle wasting pathways to maintain muscle mass, or whether there is an inhibition of signaling pathways that promote muscle atrophy, which would otherwise be activated with prolonged inactivity in non-hibernators. Addressing this question is important for the development of therapies, and whether they should inhibit muscle atrophy signaling pathways or promote muscle remodeling and hypertrophy, or attempt to do both, in order to treat muscle wasting.

As discussed in Chapter 1, the main signaling pathway that controls muscle atrophy involves the forkhead box transcription factors of the O subclass (Foxo) as well as the myogenin (MyoG) transcription factor, and their regulation of the ubiquitin proteasome system (UPS) (Moresi et al., 2010; Sandri et al., 2004; Stitt et al., 2004). The UPS is an important mechanism for protein degradation, whereby substrates are ligated to ubiquitin via E3 ubiquitin ligases like Muscle Atrophy F-Box (MAFbx/atrogin-1) and Muscle Ring Finger 1 (MURF1), which target these substrates for degradation in the proteasome (Foletta et al., 2011; Herrmann et al., 2007; Schiaffino et al., 2013). Due to the importance of both MAFbx and MURF1 for muscle atrophy, common regulators were found for both ligases. The Foxo family of transcription factors were the first of such factors (Moresi et al., 2010; Sandri et al., 2004; Stitt et al., 2004). Then, MyoG, which was initially identified as a regulator of myogenesis, was shown to be a positive regulator of both E3 ligases as well; where the expression of MAFbx and MURF1, as well as muscle atrophy were attenuated in MyoG-null mice (Moresi et al., 2010). The mammalian Foxo family has four members: Foxo1, Foxo3a, Foxo4, and Foxo6, that are involved in various cellular processes in addition to muscle atrophy, such as antioxidant defense and apoptosis (Birkenkamp \& Coffer, 2003; Greer \& Brunet, 2005; Wu \& Storey, 2014). Foxo1, Foxo3a, and Foxo4 are all regulated by the Akt/protein kinase B (PKB) signaling pathway (Figure 1.4). Specifically, Akt blocks the function of all three Foxo proteins through phosphorylation at conserved residues that 
lead to cytoplasmic localization of Foxo (Brunet et al., 1999; Matsuzaki et al., 2005; Takaishi et al., 1999; Tang et al., 1999). For Foxo4, Akt inhibits the nuclear translocation and activation of Foxo4 by phosphorylating the Threonine (Thr)-32, Serine (Ser)-197, and Ser262 residues (Matsuzaki et al., 2005; Takaishi et al., 1999). However, Foxo4 transcriptional activity has been shown to be regulated through a separate pathway, involving the Ras and Ral GTPases, as well (De Ruiter et al., 2001; Essers et al., 2004; Kops et al., 1999; Van Den Berg et al., 2013).

Ras and the Ral isoforms, RalA and RalB, are small GTPases that share very similar sequences, with Ral activation requiring the activation of Ras in order to act as signaling molecules for a variety of downstream processes like transcription, DNA synthesis, and differentiation (De Ruiter et al., 2001; Feig, Urano, \& Cantor, 1996). Ral binding protein 1 (Ralbp1) is another integral part of the Ras-Ral pathway as it acts as a downstream effector of Ral and associates with Ral in a GTP-dependent manner (Cantor, Urano, \& Feig, 1995; JullienFlores et al., 1995). Initially it was discovered that Foxo4 transcriptional activity was dependent upon activation of the Ras-Ral pathway as it results in phosphorylation of Foxo4 at Thr-447 and Thr-451 (De Ruiter et al., 2001; Kops et al., 1999) (Figure 1.5). As mentioned previously, this pathway acts independently from the control of nuclear-cytoplasmic distribution that Akt holds over Foxo4, as another downstream kinase that phosphorylates Foxo4 was found to be Stressactivated protein kinase (SAPK)/Jun amino-terminal kinase 1 (JNK1). JNK1 is bound to the cellular scaffold protein, c-Jun-amino-terminal-interacting protein 1 (JIP1), where it is activated by RalA through phosphorylation (Van Den Berg et al., 2013) (Figure 1.5). It was demonstrated that the activation of Foxo4 transcriptional activity through the Ras-Ral pathway and JNK was induced by tumor necrosis factor $\alpha(\mathrm{TNF} \alpha)$-activation of the Ras-Ral pathway (Essers et al., 2004; Van Den Berg et al., 2013). Furthermore, the induction in MAFbx expression by TNF $\alpha$ is 
reliant on Foxo4 regulation and not Foxo1/3a (Moylan, Smith, Chambers, McLoughlin, \& Reid, 2008). These previous findings stress the importance of understanding the regulation of Foxo4 through both the Ras-Ral and Akt pathways, and the importance of studying how this contributes to the control of muscle atrophy by Foxo4.

Given the unique ability of I. tridecemlineatus to avoid disuse-induced muscle wasting despite being inactive during periods of torpor and arousal during hibernation, there is a need to study the molecular mechanisms underlying muscle atrophy during hibernation in this animal. It is hypothesized that during the avoidance of muscle loss during hibernation, there will be a downregulation of MyoG- and Foxo4-mediated MAFbx and MURF1 expression, and this effect will be initiated by the Foxo4 dephosphorylation through inhibition of the Ras-Ral pathway. To test this hypothesis, the present study characterized the protein levels of total Foxo4 as well as different phosphorylated forms of Foxo4, in addition to Ras, RalA, Ralbp1, MyoG, MAFbx, and MURF1 over cycles of torpor and arousal in the skeletal muscles of I. tridecemlineatus.

\section{Materials and Methods}

\section{Animal treatment}

All animals were captured, treated and their tissues were harvested following the same protocol as previously described in Chapter 2. The skeletal muscle used was a mixture of several hind limb muscles.

\section{Total protein isolation}


Total protein extracts were prepared as previously described in Chapter 2. Samples of frozen skeletal muscle $(n=4)$ weighing approximately $0.5 \mathrm{~g}$ were used to prepare total protein extracts, which were used for western blotting. Other information as in Chapter 2.

\section{Western blotting}

Western blotting was performed as described in Chapter 2 for six time points (EC, EN, ET, LT, EA, LA) for all the proteins studied in this chapter. Equal amounts of protein from each sample (25 $\mu \mathrm{g})$ were loaded onto 8\% (Foxo4, p-Foxo4 S197, p-Foxo4 T451, Ralbp1, MURF1), $10 \%$ (MyoG, MAFbx), or 15\% (Ras and RalA) polyacrylamide gels that were run at $180 \mathrm{~V}$ for 60-120 min. Proteins were then transferred to PVDF membranes by electroblotting at $160 \mathrm{~mA}$ for $90 \mathrm{~min}$ (Foxo4, p-Foxo4, Ralbp1, MyoG, MAFbx, MURF1) or at $30 \mathrm{~V}$ for $100 \mathrm{~min}$ (Ras, RalA). Membranes were blocked after transfer for 30 min with 7.5\% (Ras, RalA, MAFbx, MURF1) or 5\% (Foxo4, p-Foxo4, MyoG, Ralbp1) w:v milk in 1x TBST. After washing, membranes were probed with specific primary antibodies at $4^{\circ} \mathrm{C}$ overnight.

Antibodies specific for mammalian Foxo4 (CS 9472) from Cell Signaling Technology (Danvers, MA), MAFbx/atrogin-1/FBXO32 (SC27645), MyoG (SC576) and p-Foxo4 S197 (SC101628) from Santa Cruz Biotechnology (Dallas, TX), MURF1/TRIM63 (GTX110475), Ras (GTX132480) and RalA (GTX114204) from Genetex (Irving, CA), as well as p-Foxo4 T451 (12053) and Ralbp1 (38202) from Signalway Antibody (Baltimore, MD) were purchased and used at a 1:1000 v:v dilution in 1x TBST. All membranes were incubated with HRP-linked antirabbit IgG secondary antibody (Bioshop: 1:6000 v:v dilution) for $30 \mathrm{~min}$ at room temperature. 
The antibodies cross-reacted with a single band on immunoblots at the expected molecular mass for the respective proteins as indicated on the antibody specification sheets.

\section{Data and Statistical Analysis}

Band densities for Foxo4, p-Foxo4, MAFbx, MURF1, Ras, Ral, and Ralbp1 on chemiluminescent immunoblots were visualized and quantified as described in Chapter 2. Data is expressed as mean \pm SEM with $\mathrm{n}=4$ independent samples from different animals.

\section{Results}

Upregulation and activation of Foxo4 at various stages during the torpor-arousal cycle

To determine the role of Foxo4 transcription factors in skeletal muscle remodeling during the torpor-arousal cycle, total expression of Foxo4 as well as relative phosphorylation of its Ser197 and Thr-451 residues was determined via western blotting at six different sampling points: EC, EN, ET, LT, EA, LA. As shown in Figure 5.1, Foxo4 protein levels increased dramatically upon entering torpor at EN (2.14-fold increase compared to EC, $\mathrm{p}<0.05)$. Foxo4 levels continued to increase until LT, where levels were 3.9-fold higher than EC ( $\mathrm{p}<0.05)$. Following LT, protein levels began to decline progressively throughout arousal. Two phosphorylated residues of Foxo4 were analyzed as well, Ser-197 and Thr-451. The Thr-451 residue of Foxo4 on its C terminus is known to be phosphorylated by JNK through activation of the Ras-Ral pathway, whereas Ser197 is phosphorylated by Akt/PKB, leading to inhibition of Foxo4 (De Ruiter et al., 2001; Essers et al., 2004; Matsuzaki et al., 2005). We observed differential phosphorylation of the two residues over the torpor-arousal cycle; where p-Foxo4 T451 levels increased dramatically upon 
entering torpor by 1.68 -fold compared to $\mathrm{EC}(\mathrm{p}<0.05)$. However, protein levels declined quickly as the squirrels progressed through torpor, where there was a $44 \%$ decrease at ET compared to EC $(\mathrm{p}<0.05)$. $\mathrm{p}$-Foxo4 T451 levels then remained low throughout the torpor-arousal cycle (Figure 5.1). On the contrary, p-Foxo4 S197 residue levels decreased immediately upon entering torpor with EN levels decreasing by $70 \%$ in comparison with EC ( $<<0.05)$. p-Foxo4 S197 levels remained low during torpor, but returned to euthermic levels during arousal (Figure 5.1).

Analysis of p-Foxo4 vs. total Foxo4 ratios

Other than analyzing protein levels of Foxo4 and two of its phosphorylated forms relative to the total protein loading control, phosphorylation ratios were calculated from the ratio of $\mathrm{p}$ Foxo to total Foxo levels. The way of interpreting the data takes changes in total protein levels into account when analyzing the changes in phosphorylation. As seen in Figure 5.2, the ratio of p-Foxo4 to total Foxo4 for the Thr-451 residue showed a progressive decrease after entering torpor, with a $77 \%$ decrease being observed at ET relative to EC ( $<<0.05)$. At LT, there was a significant rise in phosphorylation by 2-99-fold relative to ET $(\mathrm{p}<0.05)$ and the ratio remained stable after entering arousal. Similar to p-Foxo4 protein levels, the phosphorylation ratios for the Ser-197 residue differed from those of the Thr-451 residue. p-Foxo4 S197 ratios declined dramatically immediately upon entering torpor, as there was a $88 \%$ decrease in EN relative to EC $(p<0.05)$. These levels remained relatively stable throughout the rest of the torpor-arousal cycle, with increases occurring at ET and during arousal (both EA and LA) (Figure 5.2). However, these changes were not significant.

Analysis of MyoG, MAFbx, and MURF1 protein levels 
MyoG protein levels were analyzed and we observed that it remained relative stable throughout the torpor-arousal cycle with the exception of LT, where MyoG levels declined by $52 \%$ relative to $\mathrm{EC}(\mathrm{p}<0.05)$ (Figure 5.3). MAFbx levels declined progressively upon entering torpor, with a significant decrease occurring at ET $(42 \%$ decrease relative to EC, $\mathrm{p}<0.05)$ and again at LT $(69 \%$ decline relative to $\mathrm{EC}, \mathrm{p}<0.05)$. Afterwards, MAFbx returned to similar levels as ET during arousal (Figure 5.3). MURF1 showed different expression patterns from MAFbx even though they are both E3 ubiquitin ligases that target proteins for degradation (Foletta et al., 2011; Lecker, 2003; Schiaffino et al., 2013). MURF1 levels also declined during ET by $40 \%$ in comparison with EC $(\mathrm{p}<0.05)$. However, there was a 2.66-fold increase at LT relative to ET $(\mathrm{p}<0.05)$. This is spike in MURF1 levels was followed by another significant decline by $60 \%$ from LT to EA $(p<0.05)$. During late arousal, protein levels returned to euthermic levels (Figure 5.3). The MAFbx timecourse western blots were done by Dr. Shannon N. Tessier and are unpublished and not part of her thesis, she contributed her data on this target as it was vital to the present chapter and she is a co-author on our submitted manuscript for publication.

\section{Analysis of the Ras-Ral pathway}

Ras-Ral signaling has been identified as a regulator of Foxo4 through phosphorylation, and it involves two small GTPases (Ras and Ral) and possibly Ralbp1 (De Ruiter et al., 2001; Essers et al., 2004; Neel et al., 2011). The protein expression of the three above mentioned proteins were characterized. As shown on Figure 5.4, we observed a dramatic decline in Ras immediately upon entering torpor, with levels declining by $93 \%$ from $\mathrm{EC}$ to $\mathrm{EN}(\mathrm{p}<0.05)$. These levels remained stable throughout the torpor-arousal cycle. On the other hand, RalA levels increased significantly at EN from EC by 1.65 -fold $(\mathrm{p}<0.05)$. Afterwards, protein expression 
decreased significantly at ET by $64 \%$ relative to EN $(p<0.05)$, and these levels remained relatively stable over the rest of the torpor-arousal cycle (Figure 5.4). Ralbp1 expression increased dramatically as well upon entering torpor by 2.61 -fold relative to $\mathrm{EC}(\mathrm{p}<0.05)$. At LT, there was a significant decrease in protein levels back to euthermic levels (54\% relative to EC, $\mathrm{p}<0.05)$. Afterwards, Ralbp1 expression increased again during arousal, with a 2.33 -fold increase being observed at EA relative to LT ( $<<0.05)$ (Figure 5.4)

\section{Discussion}

The present study furthers our understanding of the molecular basis behind muscle remodeling and preservation during hibernation in I. tridecemlineatus. Previous studies have shown that during hibernation, pathways that promote muscle hypertrophy and muscle fiber shifting towards oxidative slow fibers are active, contributing to the preservation of total muscle mass and fiber typing (Xu et al., 2013; Zhang \& Storey, 2015). Therefore, we sought to address the question of whether molecular pathways that promote muscle atrophy are inhibited during hibernation, or if they are still upregulated as a result of inactivity but are balanced by the above mentioned pathways. Due to the role of the Foxo family of transcription factors and MyoG as central regulators of muscle atrophy via the UPS, we characterized the expression MyoG, Foxo4, as well as MAFbx and MURF1; the E3 ubiquitin ligases that are regulated by these transcription factors (Moresi et al., 2010; Sandri et al., 2004; Stitt et al., 2004). Previous work has shown that total Foxo3a protein levels as well as levels of phosphorylated Foxo3a (p-Foxo3a) at Serine 253 (S253) are upregulated throughout the torpor-arousal cycle, peaking at LT (Wu \& Storey, 2014). When taking into consideration the ratio of p-Foxo3a/total Foxo3a, there is actually no change in the phosphorylation status of Foxo3a. Also, phosphorylation of Foxo3a at the Ser-253 residue 
inhibits Foxo3a from translocating to the nucleus and regulating gene expression (Brunet et al., 1999; Dobson et al., 2011). Therefore, the increase in p-Foxo3a at the Ser-253 residue suggests that although there is upregulation of Foxo protein levels, most if not all of these additional Foxo3a proteins are inactive. Therefore, there is a need to further study the signaling pathways regulating muscle atrophy during hibernation, specifically ubiquitin ligases regulated by Foxo transcription factors.

In this study, protein level analysis was conducted on MAFbx, MURF1, Foxo4, and MyoG in addition to the main factors of the Ras-Ral pathway; including Ras, RalA, and Ralbp1. After studying the UPS, it was found that during torpor there were decreased protein levels of both MAFbx and MURF1, two E3 ubiquitin ligases that are regulated by Foxo1, 3a, 4, and are essential to muscle atrophy (Sandri et al., 2004; Stitt et al., 2004; Waddell et al., 2008). MAFbx protein levels progressively declined through torpor, with the greatest decrease occurring at LT (69\% relative to EC, $\mathrm{p}<0.05$ ). MURF1 levels also decreased at ET by $40 \%$ compared to EC $(\mathrm{p}<0.05)$ but its levels rose dramatically at LT (Figure 5.3), suggesting that these two ligases could be differentially regulated by the same or different transcription factors. One such transcription factor that is known to regulate the expression of both ligases is MyoG, and its pattern of protein expression resembles that of MAFbx during the torpor-arousal cycle, with a significant decrease in MyoG levels occurring at LT (Figure 5.3) (Bricceno et al., 2012;

MacPherson, Wang, \& Goldman, 2011; Moresi et al., 2010). It has been observed that inhibiting MyoG through the use of trichostatin A results in reduced muscle wasting, and there is a greater decline in MAFbx expression in comparison with MURF1 expression, suggesting that MyoG may regulate the expression of MAFbx to a greater extent than MURF1 Bricceno et al., 2012). 
Another important regulator of both MAFbx and MURF1 is Foxo4, and total levels of this protein significantly increased from EC and remained elevated throughout the torpor-arousal cycle, peaking at LT, where there was a 3.9-fold increase in comparison with $\mathrm{EC}(\mathrm{p}<0.05)$ (Figure 5.1). p-Foxo4 levels were also assessed, and we found differential expression of the two p-Foxo4 residues, Serine 197 (S197) and Threonine 451 (Thr451). p-Foxo4 S197 protein levels decreased by $70 \%$ while entering torpor $(\mathrm{EN})$ in comparison with $\mathrm{EC}(\mathrm{p}<0.05)$. On the other hand, at the same time point, p-Foxo4 T451 levels increased by 1.68 -fold relative to EC $(p<0.05)$. The ratios of $p$-Foxo4 to total Foxo4 were also calculated in order to accurately assess Foxo4 activity and phosphorylation status; taking into account changes in total protein levels (Zhang et al., 2010). The results indicate that there are differences in phosphorylation status between the two residues as well, with p-Foxo S197 showing decreased phosphorylation throughout torpor. However, a decrease in phosphorylation status is only seen at ET for p-Foxo4 T451 (77\% decrease relative to EC, $\mathrm{p}<0.05)$ (Figure 5.2).

The explanation for the differential phosphorylation at these residues is that the Ser-197 residue is phosphorylated by Akt/PKB, a commonly known inhibitor of Foxo4, whereas the Thr451 residue is phosphorylated by JNK1 through the Ras-Ral pathway, resulting in transcriptional activation of Foxo4 (Figure 1.5) (De Ruiter et al., 2001; Essers et al., 2004; Kops et al., 1999; Matsuzaki et al., 2005; Takaishi et al., 1999). Findings from this study indicated that the Ras protein declined immediately upon entering torpor, whereas RalA and its effector protein, Ralbp1, were initially upregulated during EN, then levels of both proteins progressively declined throughout torpor, reaching levels that were $41 \%$ and $54 \%$ that of EC for RalA and Ralbp1, respectively (Figure 5.4). Furthermore, this progressive downregulation of the Ral pathway during torpor mirrors the decline in activated JNK, or p-JNK1 (Thr-183/Tyr-185), levels 
throughout torpor, which was found in previous work from our lab (Wu \& Storey, 2014). As mentioned previously, active RalA leads to phosphorylation and activation of JNK1 that is bound to JIP1. This occurs through the accumulation of reactive oxygen species (ROS), resulting in TNF $\alpha$-mediated activation of the Ras-Ral pathway (Essers et al., 2004; Van Den Berg et al., 2013). Given that in I. tridecemlineatus, ROS production is positively associated with $T_{b}$ changes, this explains why the Ras-Ral pathway and p-JNK levels decline over torpor, as $\mathrm{T}_{\mathrm{b}}$ continues to decrease (Brown, Chung, Belgrave, \& Staples, 2012).

In summary, these results indicate that although there are significant increases in total Foxo4 protein levels throughout torpor and early arousal, the ratio of dephosphorylated Foxo4 (S197) able to translocate to the nucleus and regulate transcription, is significantly decreased. Also, since the phosphorylation ratio of p-Foxo4 (T451) is significantly decreased at ET due to inactivation of the Ras-Ral pathway and JNK, the amount of active nuclear Foxo4 able to regulate the expression of target genes like MAFbx and MURF1 is significantly reduced as well. Therefore, we can conclude that although Foxo4 is upregulated during torpor, the reduction in Foxo4 activity is more significant during torpor, especially at ET. This finding, along with the downregulation of MyoG during torpor, leads to the decreased expression of MAFbx and MURF1. This mechanism would result in reduced muscle atrophy during torpor despite mechanical unloading, so it may contribute to the preservation of muscle mass while ground squirrels hibernate. Therefore, in addition to upregulating pathways to promote hypertrophy and fiber type switching, signaling pathways regulating muscle atrophy, such as the Ras-Ral pathway, are downregulated during hibernation (Xu et al., 2013; Zhang \& Storey, 2015). These novel findings on this unique and natural physiological process in ground squirrels advances our knowledge of skeletal muscle remodeling, and they could be applied for therapeutic intervention 
in treating muscle wasting diseases like Spinal Muscular Atrophy and Duchenne Muscular Dystrophy. Specifically, our findings suggest that in addition to testing agents that promote muscle growth, like Naloxone - an NFAT activator, there needs to be an emphasis on testing inhibitors of muscle atrophy signaling pathways. For example, farnesyltransferase inhibitors designed to block the Ras-Ral pathway for anticancer treatment could also be tested for the possibility of inhibiting Foxo4 and reducing muscle atrophy (Yeh \& Der, 2007). 


\section{Figure}

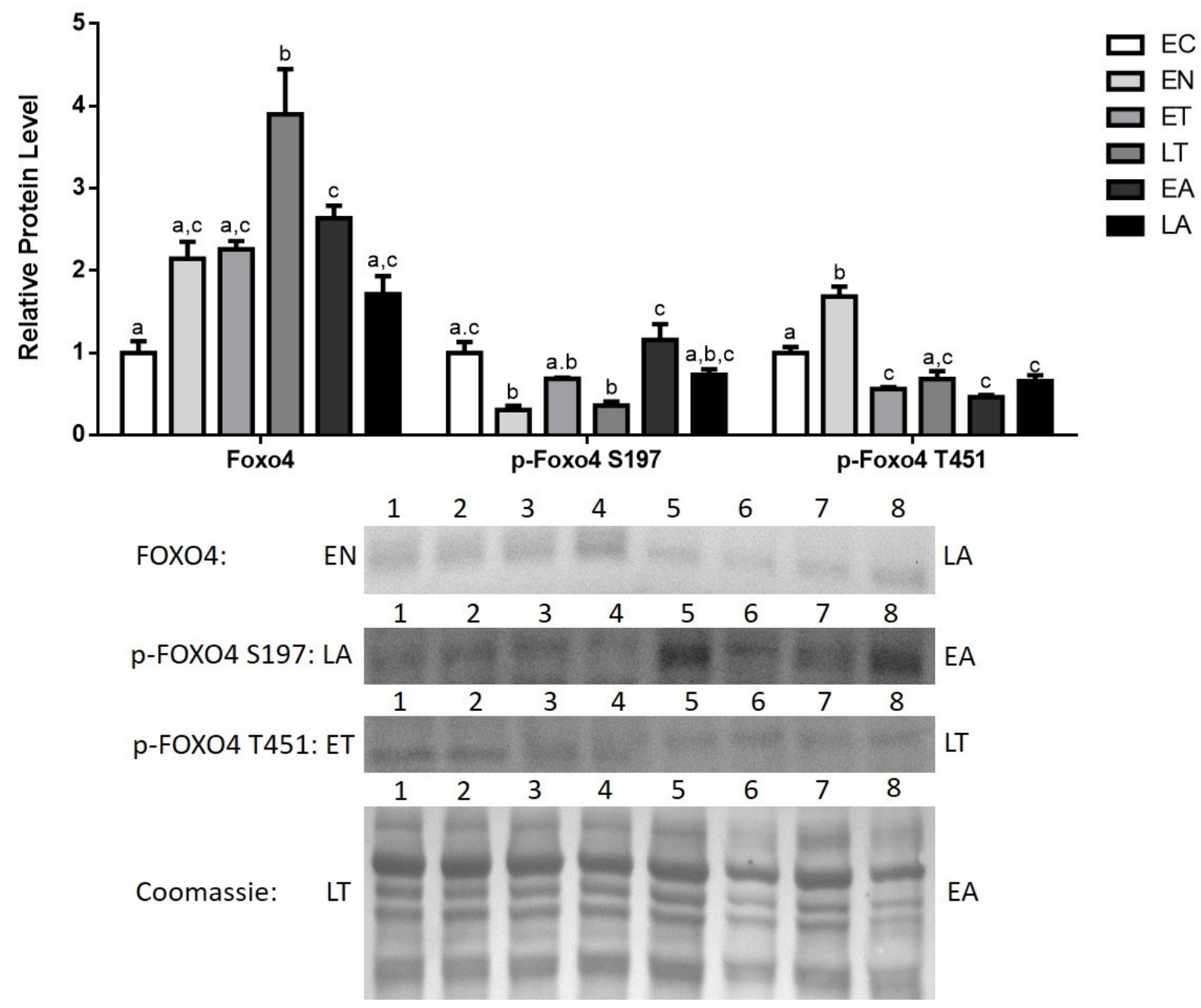

Figure 5.1: Changes in the protein levels of the Foxo4 transcription factors and its

phosphorylated forms, Ser-197 (S197) and Thr-451 (T451), over the course of the torpor-arousal cycle in skeletal muscle of I. tridecemlineatus. Foxo4, p-Foxo4 S197, and T451 protein expression levels were visualized at six sampling points: EC, EN, ET, LT, EA, LA. See Chapter 1 Materials and Methods and for more extensive definitions. Other information as in Figure 3.2. 


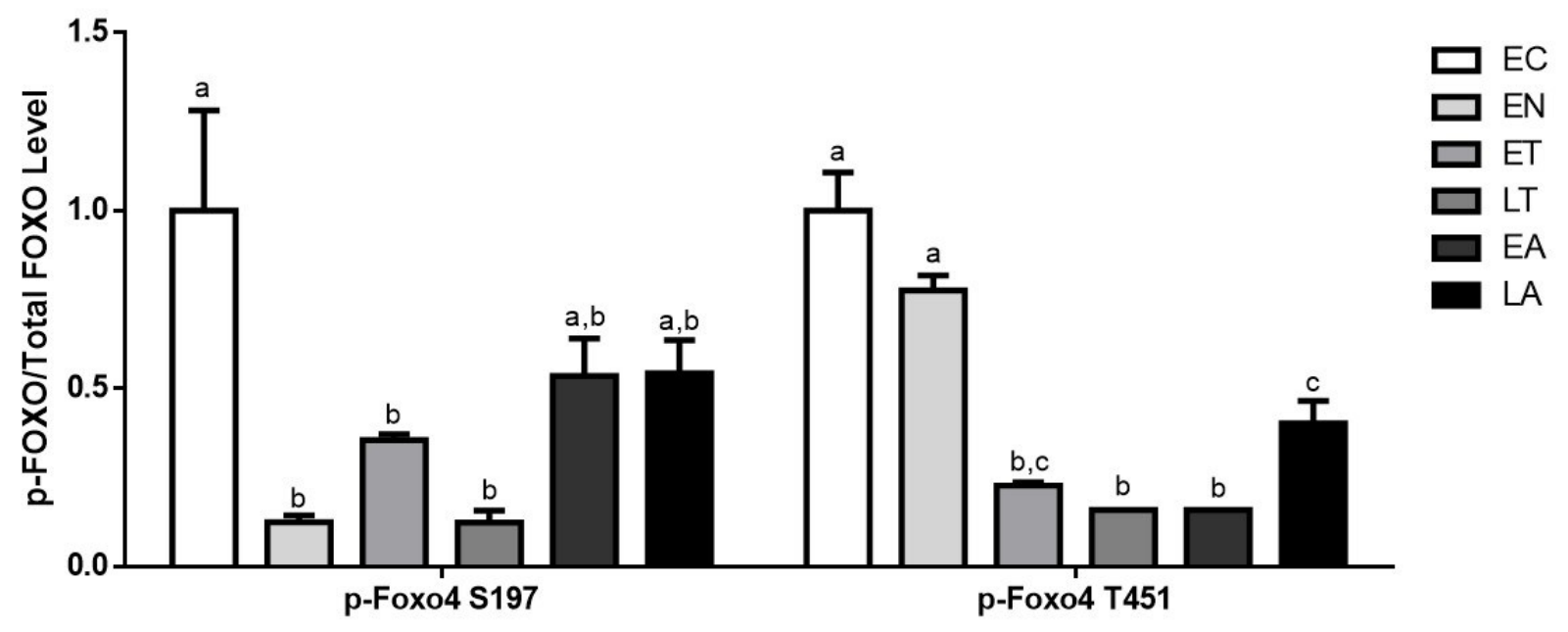

Figure 5.2: Changes in phosphorylation ratios for phosphorylated Foxo4 proteins were analyzed by taking a ratio of band densitometries between p-Foxo 4 protein levels and total Foxo4 protein levels. Phosphorylation ratios were determined for p-Foxo4 S197 and T451. Other information as in Figure 5.1. 


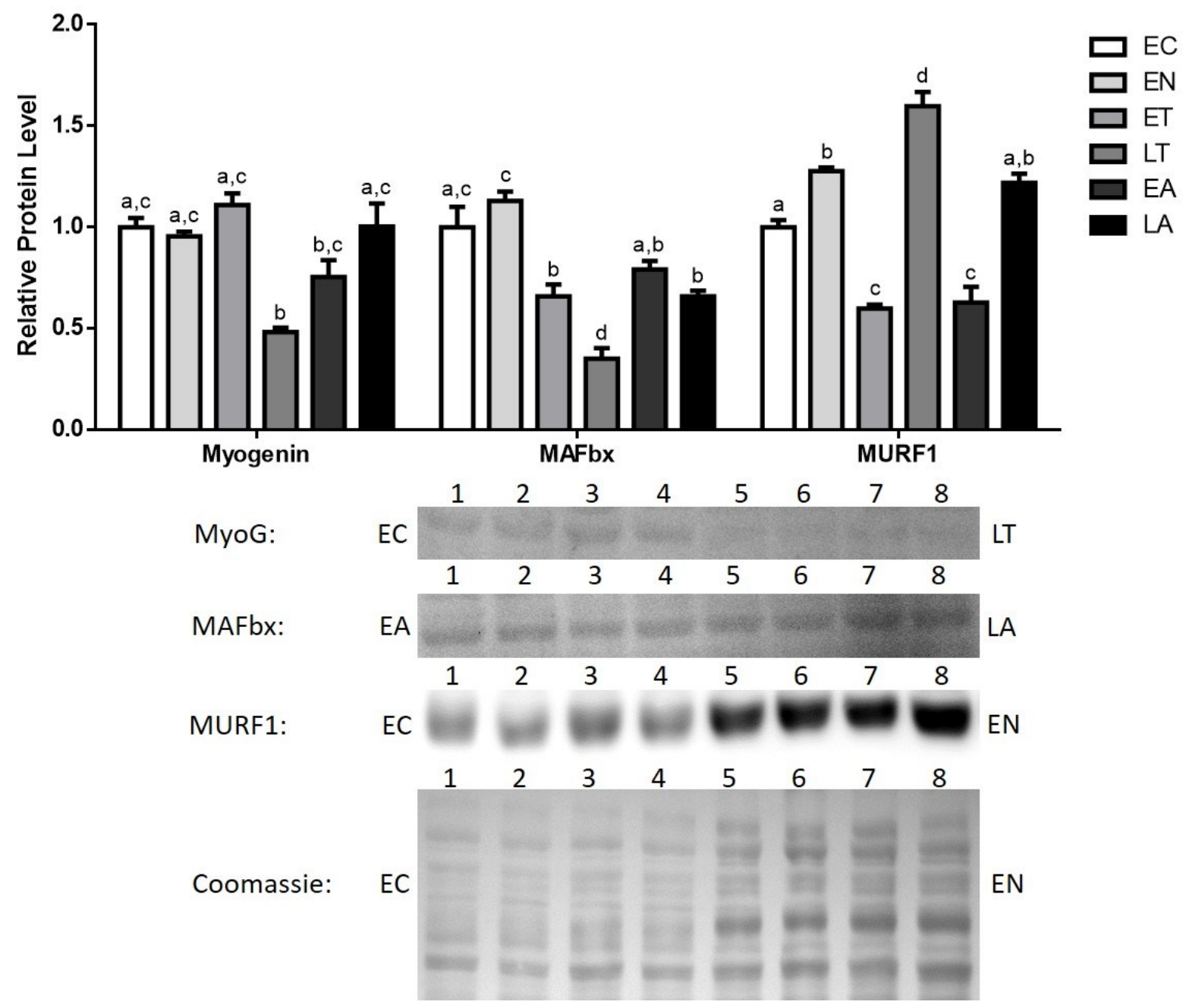

Figure 5.3: Changes in protein levels of the muscle proteins MyoG, MAFbx, and MURF1 over the course of the torpor-arousal cycle in skeletal muscle of I. tridecemlineatus. Other information as in Figure 3.2. The MAFbx timecourse western blots were done by Dr. Shannon N. Tessier and are unpublished and not part of her thesis. She contributed her data on this target as it was vital to the present chapter and she is a co-author on our submitted manuscript for publication. 


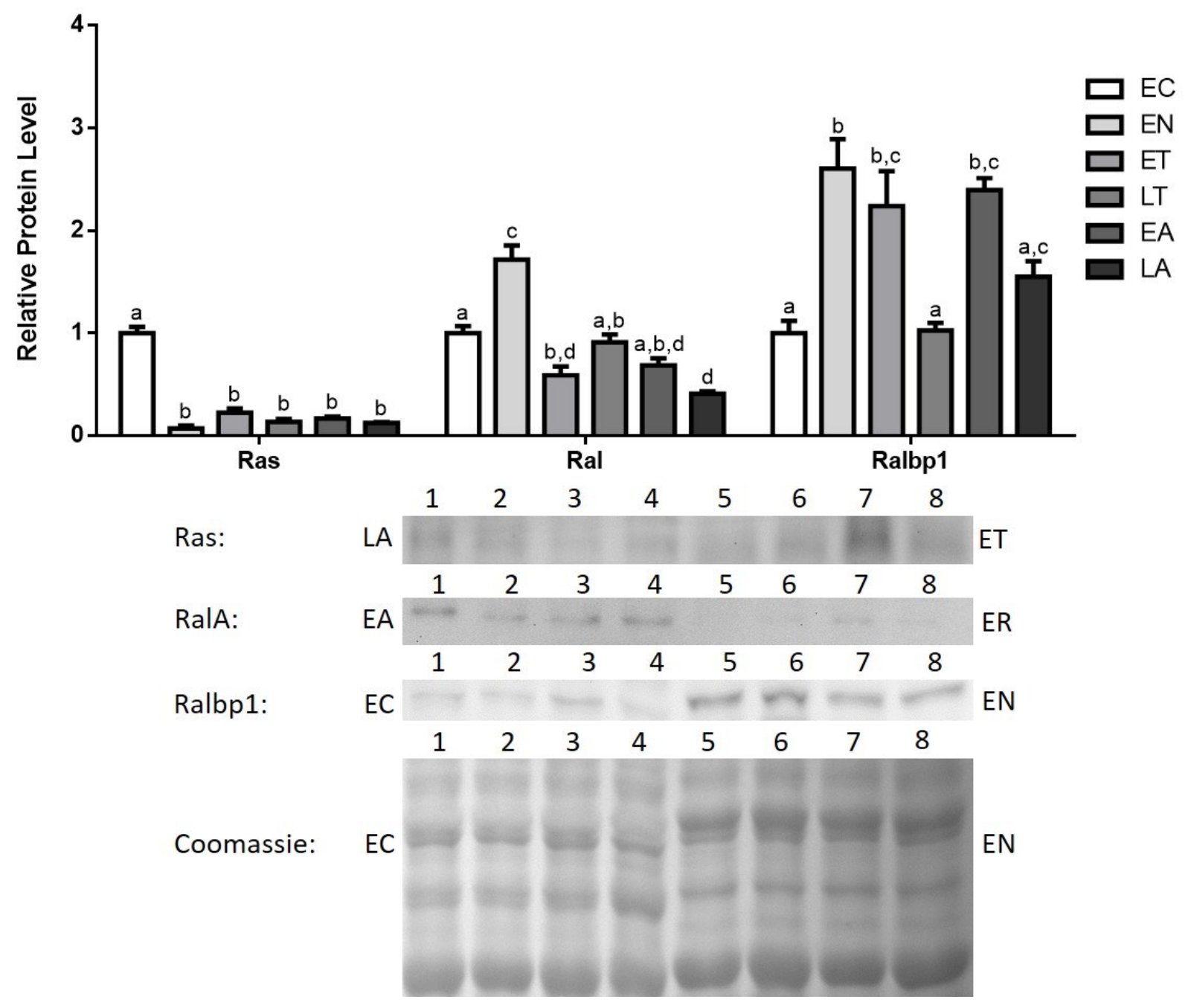

Figure 5.4: Changes in protein levels of proteins in the Ras-Ral pathway: Ras, RalA, and Ralbp1 over the course of the torpor-arousal cycle in skeletal muscle of I. tridecemlineatus. Other information as in Figure 3.2. 


\section{Chapter 6}

Transcriptional activation of muscle atrophy promotes cardiac muscle remodeling during mammalian hibernation in ground squirrels 


\section{Introduction}

As discussed in Chapters 1 and 4, each organ/tissue of the hibernating ground squirrel must make specific adjustments that allow them to maintain or readjust physiological functions at low $\mathrm{T}_{\mathrm{b}}$ values to support long term torpor. Heart rate is strongly reduced during torpor, often from euthermic rates of $350-400$ beats/min to just $5-10$ beats/min. These changes in heart rate, plus the increased viscosity of blood at low $T_{b}$ values, require adjustments to be made in squirrel heart dynamics (Frerichs \& Hallenbeck, 1998; Frerichs et al., 1994). For example, the strength of each individual contraction must be significantly increased in response to the pressure and volume overloads; as a result cardiac hypertrophy is observed (Depre et al., 2006). In most mammals, cardiac hypertrophy is characterized by significant cardiac fibrosis whereby collagen deposition stiffens cardiac chamber walls, reduces diastolic filling, and ultimately prevents the heart from pumping enough blood to meet body demands; this is a condition known as heart failure (Day, 2013). Interestingly, previous findings have shown that ground squirrels in hibernation have larger left ventricular mass, internal dimensions, and wall dimensions in comparison with active squirrels; suggesting reversible left ventricular hypertrophy is occurring (Nelson \& Rourke, 2013). This fascinating process may be related to molecular adaptations that occur during hibernation, which are just beginning to be discovered. Understanding the mechanism of reversible cardiac hypertrophy in hibernators would provide novel insight into the development and treatment of maladaptive cardiac hypertrophy and heart failure.

As mentioned previously, one of the key elements of cardiac hypertrophy is the stressinduced adaptation in protein turnover; which involves protein synthesis and degradation. Both of these mechanisms are activated by increased cardiac workload due to pressure or volume 
(Depre et al., 2006). Upregulation of NFAT has been implicated in the increased synthesis of numerous proteins during cardiac hypertrophy as shown in Chapter 4. With regard to protein degradation, the UPS is known to be an important mechanism whereby substrates are ligated to ubiquitin via ubiquitin ligases and are targeted for degradation (Herrmann et al., 2007). As discussed in Chapter 5, the specificity of the UPS is determined by E3 ubiquitin ligases that recognize specific target proteins, which include MAFbx and MURF1. The role of the UPS remains relatively unclear in cardiac muscle in comparison with skeletal muscle; although they have shown upregulation in association with cardiac hypertrophy or heart failure (Depre et al., 2006; Galasso et al., 2010). This increased in expression of ubiquitination machinery may be in response to the increase in over protein production that accompanies hypertrophy in the heart or in response to modified or damaged proteins that need to be degraded (Day, 2013). Therefore, with respect to reversible cardiac hypertrophy, we suspect that the UPS will be mostly active during late torpor or arousal, especially when coming out of hibernation as perfusion and $\mathrm{T}_{\mathrm{b}}$ will increase; thus reversing the hypertrophic stimulus and promoting atrophy instead.

As described in Chapter 5, early studies have shown that both MAFbx and MURF1 are upregulated under similar atrophy-inducing conditions, suggesting that both ligases are regulated by common TFs; Foxos were the first set of such factors to be identified (Sandri et al., 2004; Stitt et al., 2004). For example, Foxo1 was shown to increase MAFbx or MURF1 levels by blocking their inhibition from the IGF-1/PI3K/Akt insulin signaling pathway. Thus, it was initially believed that Foxo1 indirectly increases the expression of MAFbx and MURF1 (Stitt et al., 2004). However, later studies identified that Foxo TFs share consensus sequences that allow them to bind directly to the MAFbx and MURF1 promoters (Sandri et al., 2004; Waddell et al., 2008). In addition, it was also observed that not all Foxo family members bind equally to the 
promoters of MAFbx and MURF1 (Waddell et al., 2008). For instance, Foxo1 activates the Foxo binding motif; leading to MURF1 upregulation, to a greater degree than Foxo3a or 4 (Waddell et al., 2008). Akt, otherwise known as protein kinase B (PKB), has been shown to block the function of all three Foxo proteins through phosphorylation, leading to their containment in the cytoplasm (Brunet et al., 1999; Takaishi et al., 1999; Tang et al., 1999). Dephosphorylation of Foxo factors on the other hand leads to nuclear localization and growth suppression or apoptosis (Takaishi et al., 1999). Akt has numerous phosphorylation sites on Foxo1, 3a, and 4, including Threonine ${ }^{32}\left(\mathrm{Thr}^{32}\right)$ for Foxo3a, as well as $\mathrm{Thr}^{24}$ and Serine ${ }^{319}\left(\mathrm{Ser}^{319}\right)$ for Foxo1 (Dobson et al., 2011) (Figure 1.4). Aside from Akt, Foxos can also be phosphorylated and inhibited by numerous other kinases; including Jun N-terminal kinase (JNK), AMP-activated protein kinase (AMPK), cyclin-dependent kinase (CDK), and MAPK-activated protein kinase (MK) (Huang, Regan, Lou, Chen, \& Tindall, 2006; Kress et al., 2011; van der Horst \& Burgering, 2007; Yuan et al., 2008) (Figure 1.4).

Aside from Foxos, Myogenin (MyoG) has also been identified as a positive regulator of MAFbx and MURF1 (Figure 1.4); the expression of both ligases as well as muscle atrophy were attenuated in MyoG-null mice (Moresi et al., 2010). Given the unique tendencies of hibernators to undergo reversible cardiac hypertrophy over the course of the hibernation season, significant cardiac remodeling involving protein turnover is believed to occur. We hypothesize that the Foxo and MyoG TFs in addition to the E3 ligases MAFbx and MURF1 would play a significant role in this process. Therefore, the present study analyzed the protein expression of Foxol and 3a, along with their various phosphorylated forms, in addition to Foxo4, MyoG, MAFbx, and MURF1 over the torpor-arousal cycle in the cardiac muscle of thirteen-lined ground squirrels. 


\section{Materials and methods}

Animals

All animals were captured, treated and their tissues were harvested following the same protocol as previously described in Chapter 2. The cardiac tissue used was a mixture of atrial and ventricular tissue.

\section{Total Protein Extract Preparations}

Samples of frozen cardiac muscle (approximately $500 \mathrm{mg}$ ) were homogenized for total protein extraction as previously described. Total protein extracts were used for western blotting experiments as previously described in Chapter 2.

\section{Western Blotting}

Western blotting was performed as described in Chapters 2 and 4 for the following six time points: EC, EN, ET, LT, EA, IA for Foxo1, Foxo3a, their phosphorylated forms, as well as Foxo4, MyoG, MAFbx, and MURF1. Sample aliquots containing $25 \mu \mathrm{g}$ of protein were loaded onto 8\% [FOXO1, 3, 4, p-FOXO1 (T24), p-FOXO3 (T32), p-FOXO1 (S319), p-FOXO3a (S318/321), MURF1] or 10\% (MyoG, MAFbx) polyacrylamide gels and were run at $180 \mathrm{~V}$ for 60-120 min. Proteins were then wet transferred to PVDF membranes by electroblotting at 160 mA for $1.5 \mathrm{~h}$. Membranes were blocked for 30 min with 5\% (for Foxo1, 3a, p-Foxo1, p-Foxo3a) or 7.5\% (Foxo4, MyoG, MAFbx, MURF1) w:v milk in 1x TBST. After washing, membranes were probed with specific primary antibodies at $4^{\circ} \mathrm{C}$ overnight. 
The MyoG, MAFbx, MURF1, Foxo4 primary antibodies were the same as those used in Chapter 5. Other primary antibodies used were: rabbit polyclonal FOXO1 (gtx110724), FOXO3a (gtx 100277), p-FOXO1/FKHR Ser ${ }^{319}$ (Genescript A00373), p-FOXO3a Ser ${ }^{318 / 321}$ (cs 9465), and p-FOXO1 $\mathrm{Thr}^{24} / \mathrm{p}-\mathrm{FOXO} 3 \mathrm{a} \mathrm{Thr}{ }^{32}$ (cs 9464P). All antibodies were used at a 1:1000 v:v dilution in 1x TBST. All blots were then incubated with HRP-linked anti-rabbit IgG secondary antibody (Bioshop: 1:6000 v:v dilution) for $30 \mathrm{~min}$ at room temperature. The antibodies cross-reacted with a single band on immunoblots at the expected molecular mass for the respective proteins as indicated on the antibody specification sheets. Other information as in Chapter 2.

\section{Quantification and Statistics}

Band densities for Foxo1, 3a, 4, p-Foxo1, p-Foxo3a, MyoG, MAFbx, and MURF1 were visualized and quantified as described in Chapter 2. Data are expressed as means \pm SEM, $n=4$ independent samples from different animals.

\section{Results}

Analysis of Foxol and p-Foxol protein levels

Total Foxo1 levels immediately rose by 2.3 -fold (in comparison with $\mathrm{EC}, \mathrm{p}<0.05$ ) upon entering torpor (EN) and declined during torpor (ET, LT). However, Foxo1 levels were still elevated (1.57- and 1.33-fold relative to EC, for ET and LT respectively) before decreasing during arousal (Figure 6.1). In contrast, protein levels for phosphorylated Foxol at $\mathrm{Thr}^{24}(\mathrm{p}$ Foxo1 T24), one of the inhibitory phosphorylation sites targeted by Akt (Dobson et al., 2011), declined and remained low throughout the torpor-arousal cycle $(62.6 \% .45 \%, 62 \%$, and $77 \%$ 
relative to EC, $\mathrm{p}<0.05$ for $\mathrm{EN}, \mathrm{ET}, \mathrm{LT}$, and EA respectively) and returned to baseline levels during IA (Figure 6.1). Ser ${ }^{319}$ (S319) is another inhibitory phosphorylation site on Foxo1 that is targeted by Akt (Dobson et al., 2011), p-Foxo1 S319 levels dropped upon entry and during torpor (EN, ET, LT) by 47-54\% in comparison with EC ( $\mathrm{p}<0.05)$. Part of the Foxo1 and p-Foxo1 S319 data was contributed by Oscar Aguilar, a former student in a lab who began to study these targets in ground squirrel but did not finish or write up this work.

Analysis of Foxo3a and p-Foxo3a protein levels

Total Foxo3a levels increased dramatically at EN and remained elevated throughout the torpor-arousal cycle, with the peak occurring at LT (4.46-fold in comparison with EC, $\mathrm{p}<0.05$ ) (Figure 6.2). Akt is known to inhibit Foxo3a from translocating to the nucleus and regulating the transcription of genes by phosphorylating Foxo3a at T32 (Dobson et al., 2011). p-Foxo3a T32 protein levels decreased dramatically by $92 \%$ relative to $\mathrm{EC}(\mathrm{p}<0.05)$ at $\mathrm{EN}$, and although protein levels rose throughout torpor and during EA, the levels were still significantly decreased in comparison with EC ( $74 \%, 66 \%, 41 \%$ for ET, $\mathrm{LT}$, and EA respectively, $\mathrm{p}<0.05)$ (Figure 6.2). p-Foxo3a S318,321 targets phosphorylation sites that are unrelated to the phosphorylation sites targeted by common Foxo3a inhibitory proteins like Akt and JNK (Fig. 1) (Dobson et al., 2011). p-Foxo3a S318, 321 protein levels remained steady throughout most of the torpor-arousal cycle, but it increased dramatically during ET ( 2.39 -fold increase relative to EC, $p<0.05)$, contrasting the trend observed with the other p-Foxo proteins. Part of the total Foxo3a data was contributed by Oscar Aguilar.

Analysis of p-Foxo vs. total Foxo ratios 
Aside from analyzing protein levels of Foxo1, 3a, and their phosphorylated forms relative to the Coomassie blue total protein loading control, phosphorylation ratios were also obtained by calculating and plotting the ratio of p-Foxo to total Foxo protein levels. Visualizing the data in this manner takes into account changes in total protein levels relative to changes in phosphorylated protein level. The p-Foxo1 S319/Foxo1 ratio showed a significant decline upon entering and throughout torpor $(80 \%, 69 \%, 63 \%$ relative to EC for EN, ET, LT respectively, $\mathrm{p}<0.05$ ) (Figure 6.3). The $\mathrm{p}$-Foxo1 T24/Foxo1 and the p-Foxo3a T32/Foxo3a showed similar patterns for their respective phosphorylation ratios, with dramatic declines in phosphorylation upon entering torpor, which was sustained throughout the torpor arousal cycle (Figure 6.3). The phosphorylation ratios decreased by $86 \%$ during EN for p-Foxo1/Foxo1 and by $97 \%$ for $\mathrm{p}$ Foxo3a/Foxo3a with respect to EC ( $<<0.05)$. The phosphorylation ratio for $p$-Foxo3a S318/321 declined during $\mathrm{EN}$ as well ( $76 \%$ relative to $\mathrm{EC}, \mathrm{p}<0.05)$, but then the ratio increased immediately afterwards during ET (2.85-fold relative to EN, p<0.05) (Figure 6.3).

Analysis of Foxo4 and MyoG protein levels

Foxo4 protein levels remained constant throughout the torpor-arousal cycle with the exception of LT, where Foxo4 levels peaked and were significantly elevated in comparison with EN (2.73-fold in comparison with EN, p<0.05) (Figure 6.4). MyoG protein levels exhibited the same pattern of expression as Foxo4, where MyoG levels remained fairly constant throughout the torpor-arousal cycle except for LT, where MyoG levels spiked (2.44-fold relative to EC, $\mathrm{p}<0.05)$ (Figure 6.4).

Analysis of MAFbx and MURF1 protein levels 
MAFbx protein levels remained fairly stable during EN and ET, and then suddenly spiked during LT (3.2-fold increase in comparison with EC, $\mathrm{p}<0.05$ ). Afterwards, MAFbx levels remained fairly high during EA and IA (1.98- and 2.45-fold increases relative to EC for EA and IA respectively, $\mathrm{p}<0.05$ ) (Figure 6.5 ). MURF1 protein levels also remained fairly constant during EN and ET, then it began increasing during LT and peaked during EA (1.5- and 1.8-fold increases in comparison with $\mathrm{EC}, \mathrm{p}<0.05$ ) (Figure 6.5).

\section{Discussion}

The present chapter aimed at furthering our understanding of the molecular mechanisms underlying muscle remodeling in cardiac muscle during hibernation in the 13-lined ground squirrel (13LGS). The ground squirrel is an excellent model for studying the process of cardiac hypertrophy as the animal enlarges its heart when its $T_{b}$ falls to $4^{\circ} \mathrm{C}$ because increased contractility is needed to pump colder, and more viscous blood throughout the body (Frerichs \& Hallenbeck, 1998; Frerichs et al., 1994; Nelson \& Rourke, 2013). However, when bouts of the torpor-arousal cycle end and squirrels come out of hibernation, the heart return to its regular size; hence the process of cardiac hypertrophy is reversed following hibernation (Nelson \& Rourke, 2013). Therefore, the present study focused on elucidating the mechanism behind the reversible cardiac hypertrophy that occurs in ground squirrels. With regards to reversing cardiac hypertrophy, we hypothesized that the family of TFs known as Foxos play a significant role in this process. Foxos control the ubiquitin/proteasome system (UPS), which is responsible for protein degradation, through its regulation of the E3 ubiquitin ligases MAFbx and MURF1 (Figure 1.4) (Sandri et al., 2004; Stitt et al., 2004). 
With regard to the UPS, our data demonstrates that there is an activation of this system during LT and arousal, leading to protein degradation and muscle atrophy. MAFbx and MURF1 both showed significant increases during LT and EA by as much as 3.2-fold from EC $(\mathrm{p}<0.05)$ (Figure 6.5). These results support the hypothesis that protein degradation and atrophy of the heart occurs naturally during arousal because squirrels are recovering from the physiological and environmental stresses that caused enlargement of their hearts (Nelson \& Rourke, 2013; Yan et al., 2015).

In addition, under conditions of environmental stress such as glucose deprivation and oxidative damage similar to those experienced by the 13LGS during hibernation, the expression of ubiquitin ligases are increased through Foxo signaling (Paula-Gomes et al., 2013). For example, TNF $\alpha$-mediated activation of Foxo4 occurs as a result of ROS production. In $I$. tridecemlineatus, Ros production increases as $\mathrm{T}_{\mathrm{b}}$ is increasing following $\mathrm{LT}$ during arousal (Brown et al., 2012). Therefore, our data demonstrates that there are significant upregulations in two regulators of the E3 ligases, Foxo4 and MyoG (Moresi et al., 2010; Moylan et al., 2008; Waddell et al., 2008). Protein expression of these two TFs showed the same pattern of significant upregulation during LT, where Foxo4 increased 2.73-fold $(\mathrm{p}<0.05)$ and MyoG increased 2.44fold relative to EC $(\mathrm{p}<0.05)$ (Figure 6.4). This data also supports the idea that upregulation of these positive regulators of MAFbx and MURF1 during LT initiates the rise in MAFbx and MURF1 protein levels during LT and arousal - leading to atrophy and protein degradation in the heart.

It should be noted that Foxo1 and 3a showed increased expression and activity earlier during the torpor-arousal cycle than did Foxo4 and MyoG. Foxo1 protein levels peaked at EN 
(2.3-fold relative to EC, $\mathrm{p}<0.05$ ) (Figure 6.1). Foxo3a on the other hand showed very high levels throughout the torpor-arousal cycle with the exception of EC (Figure 6.2). Furthermore, the Foxo1 amino acid residues $\mathrm{Ser}^{319}$ and $\mathrm{Thr}^{24}$ are sites that $\mathrm{Akt} / \mathrm{PKB}$ can phosphorylate in order to prevent the nuclear translocation of Foxo1; thus preventing its regulation of downstream targets. $\mathrm{Thr}^{32}$ is an Akt phosphorylation site on Foxo3a that plays the identical role (Dobson et al., 2011). Phosphorylation ratios of p-Foxo1 $\mathrm{Thr}^{24}$ and $\mathrm{Ser}^{319}$ both increased by at least a $63 \%$ decreases from EC $(\mathrm{p}<0.05)$ during torpor. For Foxo3a, the phosphorylation ratios of $\mathrm{Thr}^{32}$ - an inhibitory Foxo3a site, and $\mathrm{Ser}^{318 / 321}$ - a non-inhibitory phosphorylation site, were analyzed as well. Our results show that $\mathrm{p}$-Foxo3a $\mathrm{Thr}^{32}$ ratios decreased by over $78 \%$ from $\mathrm{EC}(\mathrm{p}<0.05)$ throughout the torpor arousal cycle, whereas decreases in the ratio of $\mathrm{Ser}^{318 / 321}$ increased during torpor (2.85fold at ET relative to EN, $\mathrm{p}<0.05$ ) (Figure 6.3). Therefore, we conclude that there was not only an increase in Foxo1 and Foxo3a levels during torpor, but there is also a large elevation in Foxo1 and 3a activity, as defined by an increase in the amount of dephosphorylated or nuclear Foxo. Since p-Foxo3a $\operatorname{Ser}^{319}$ was not an inhibitory phosphorylation site targeted by Akt, it follows that phosphorylation ratios would show less pronounced decreases than those for phosphorylation sites targeted by Akt and a different pattern of expression during the torpor-arousal cycle.

Therefore, in the context of reversing cardiac hypertrophy during hibernation, it is likely that Foxo4 and MyoG are important factors that regulate this process. They are the main activators of the UPS during hibernation through upregulation of the ligases, MAFbx and MURF1, throughout arousal. Foxo1 and 3a are other factors believed to regulate the expression of MAFbx and MURF1 but they have also been implicated in a vast number of cellular processes in addition to protein degradation; these processes include cell cycle inhibition, anti-oxidative response, and a shift in cellular metabolism away from anabolic processes towards catabolic 
metabolism (Eijkelenboom \& Burgering, 2013; Tessier \& Storey, 2016; van der Horst \& Burgering, 2007; Wu \& Storey, 2014). These processes have been shown to be activated during hibernation in 13LGS (Wu \& Storey, 2012, 2014). Therefore, Foxo1 and 3a could be expressed earlier during the torpor-arousal cycle due to its important role in regulating functions other than atrophy. This hypothesis could also explain the high levels of Foxol and 3a expression and activity during IA despite a decline in MURF1 expression ( $77 \%$ in comparison to EA, $\mathrm{p}<0.05$ ). During arousal, there is a dramatic rise in reactive oxygen species (ROS) production associated with oxidative thermogenesis to rewarm the body following torpor (Osborne \& Hashimoto, 2006). In order to resist oxidative damage, Foxol and 3a must increase or maintain high levels of expression and activity late during arousal.

In conclusion, the present study provides insight into some of the important proteins involved in cardiac muscle remodeling during hibernation. The results from this study indicate that there is an upregulation of the E3 ligases MAFbx and MURF1 in addition to their regulators, mainly Foxo4 and MyoG in a coordinated fashion during late torpor and throughout arousal. The coordination of Foxo4, MyoG, MAFbx, and MURF1 expression suggests that Foxo4 and MyoG may be regulators that are specific to the reversal of cardiac hypertrophy, whereas Foxo1 and 3a primarily regulate other processes such as anti-oxidant response in the heart. Therefore, in our animals, the increase in expression of the ubiquitination machinery may occur during torpor, albeit late, in response to an increase in protein synthesis from the hypertrophic response being activated in the heart during early torpor (Tessier \& Storey, 2012; Zhang \& Storey, 2015). The results provide support for the idea that cardiac hypertrophy causes activation of the UPS to reduce cardiac mass and dimensions, thus reversing cardiac hypertrophy. The molecular basis of cardiac hypertrophy occurring naturally in the hearts of 13LGS could mimic the conditions of 
maladaptive cardiac hypertrophy and heart failure that occurs in a clinical setting, and much can be learned from how this hibernator model reverses cardiac hypertrophy so efficiently and naturally (Nelson \& Rourke, 2013). 


\section{Figures}
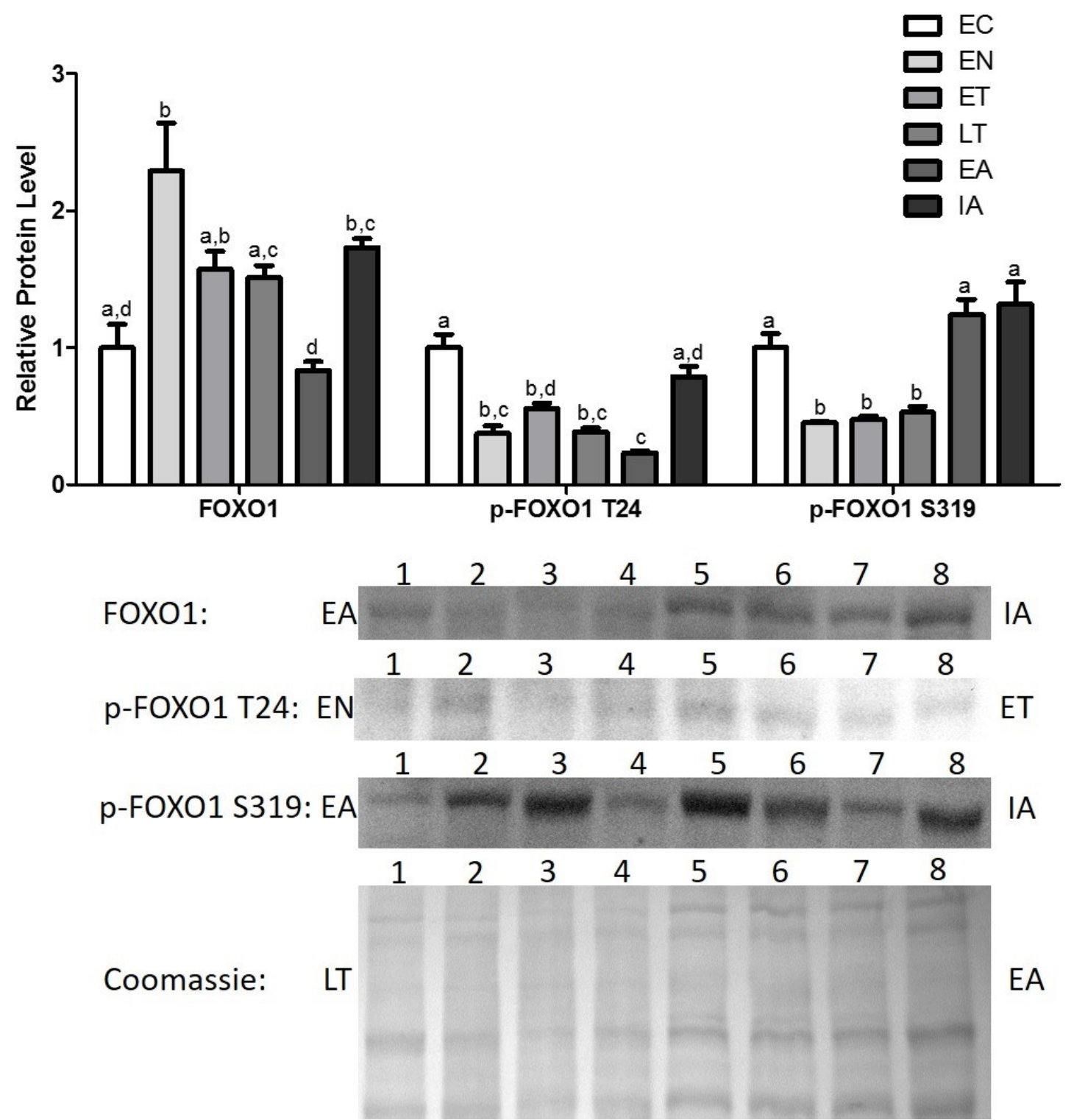

Figure 6.1: Changes in the protein levels of the Foxo1 TF and its phosphorylated forms $\mathrm{Ser}^{319}$ (S319) and $\mathrm{Thr}^{24}$ (T24) over the course of the torpor-arousal cycle in cardiac muscle of $I$. tridecemlineatus. Foxo1, p-Foxo1 S319, and T24 protein expression levels were visualized at six sampling points: EC, EN, ET, LT, EA, and IA. See Chapter 2 as well as Figure 3.2 for more information. Part of the Foxo1 and p-Foxo1 S319 timecourse data was contributed by Oscar Aguilar, although the bands shown were from western blots performed by me. 

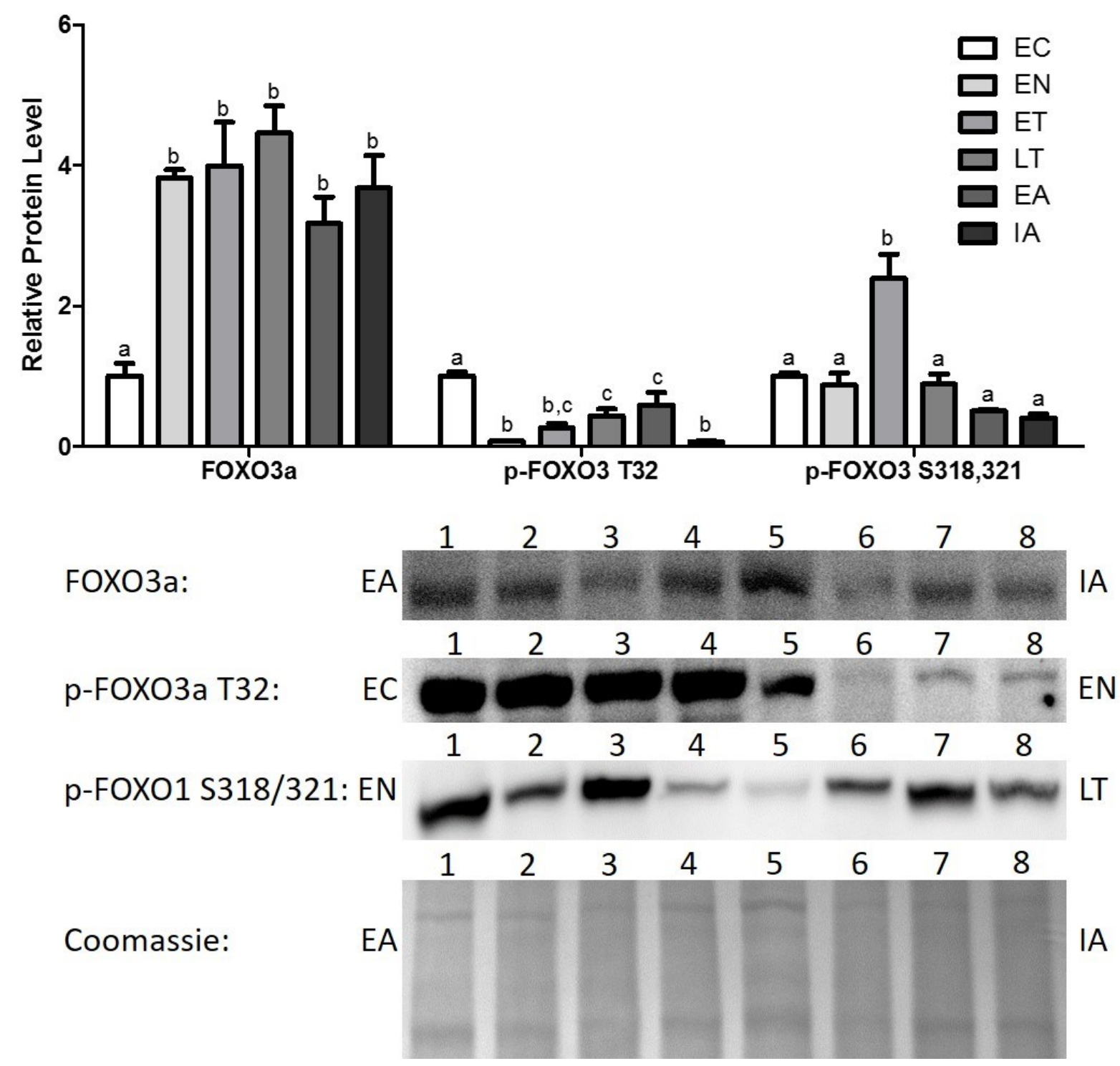

Figure 6.2: Changes in protein levels of the Foxo3a TF and its phosphorylated forms $\mathrm{Ser}^{318 / 321}$ (S318/321) and $\mathrm{Thr}^{32}$ (T32) over the course of the torpor-arousal cycle in the cardiac muscle of $I$. tridecemlineatus. Other information as in Figure 3.2. Part of the Foxo3a timecourse data was contributed by Oscar Aguilar, although the bands shown were from western blots performed by me. 


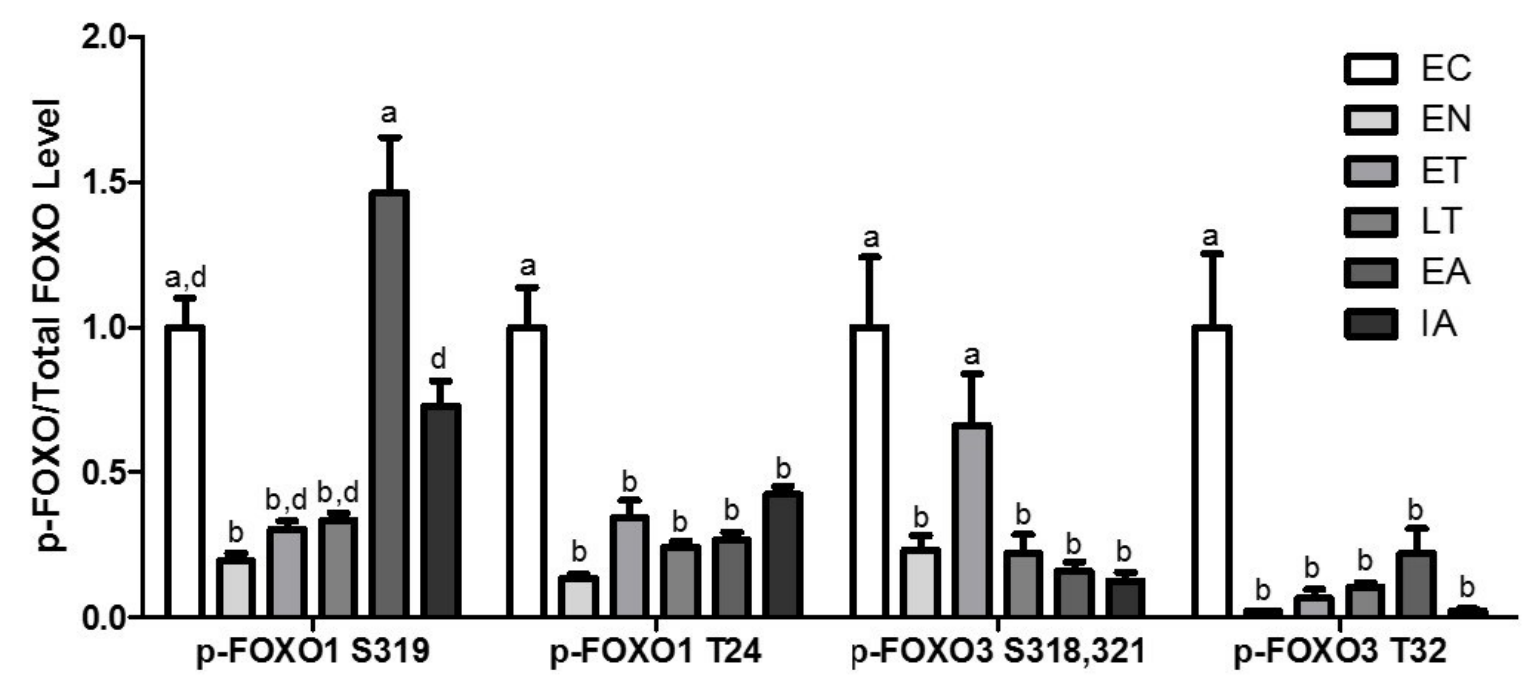

Figure 6.3: Changes in phosphorylation ratios for phosphorylated Foxo proteins were analyzed by taking a ratio of band densitometries between p-Foxo protein levels and total Foxo protein levels. Phosphorylation ratios were determined for p-Foxo1 S319, T24, p-Foxo3a S318/321, and T32. Other information as in Figure 3.2. 

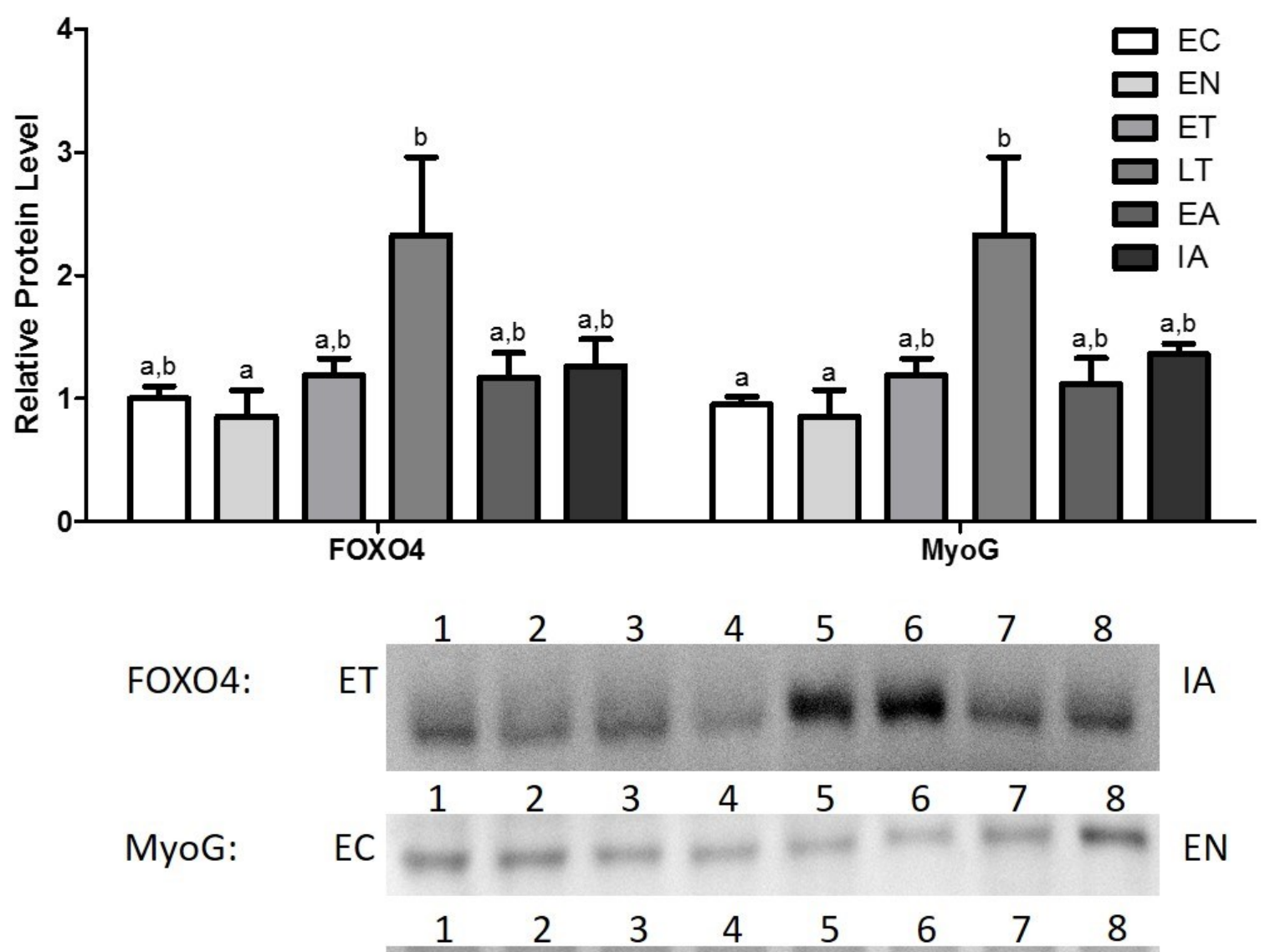

Coomassie: EC

EN

Figure 6.4: Changes in protein levels of the TFs Foxo4 and MyoG over the course of the torporarousal cycle in the cardiac muscle of I. tridecemlineatus. Other information as in Figure 3.2. 

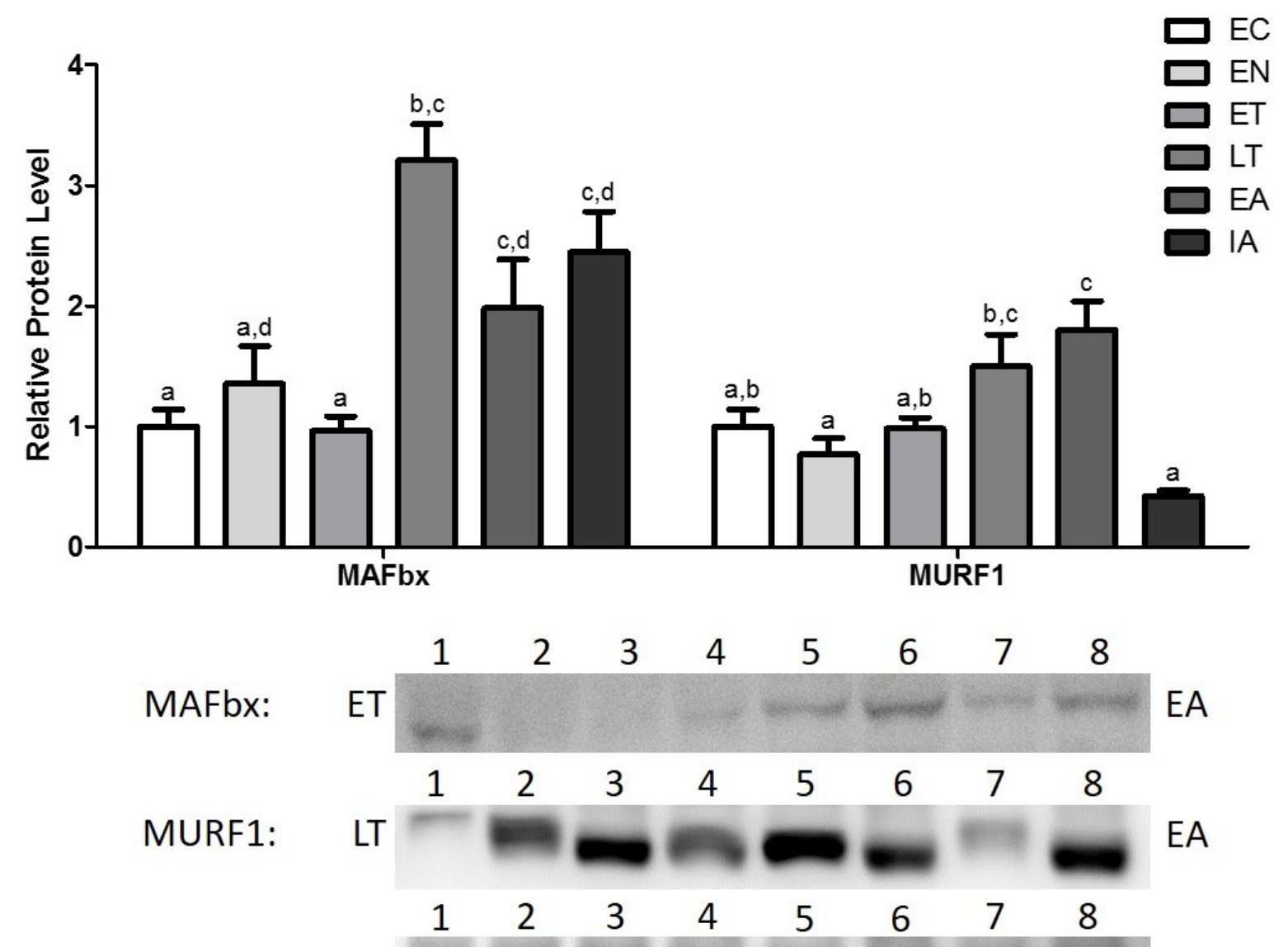

Coomassie: LT

EA

Figure 6.5: Changes in protein levels of the ubiquitin ligases MAFbx and MURF1 over the course of the torpor-arousal cycle in the cardiac muscle of I. tridecemlineatus. Other information as in Figure 3.2. 


\section{Chapter 7}

General Discussion 
Physiological adaptation to environmental changes is vital to the survival of many if not all organisms. As a result, organisms facing extreme environmental challenges have developed a range of adaptations to ensure their survival. One such adaptation used by some mammals in order to survive prolonged seasonal exposure to stressful environmental conditions (i.e. lack of food, frigid temperatures) is hibernation. The thirteen-lined ground squirrel (I. tridecemlineatus) is an excellent example of a hibernating mammal, and by studying the molecular mechanisms behind how it adapts to stress, we can learn a lot about how it copes with conditions (i.e. $\mathrm{T}_{\mathrm{b}}$ at $4^{\circ} \mathrm{C}$ during torpor) that are damaging if not lethal for nonhibernating mammals like humans. In particular, the squirrel's ability to undergo skeletal and cardiac muscle remodeling to avoid significant losses in skeletal muscle mass and to avoid maladaptive cardiac hypertrophy are especially unique. In fact, many studies have shown the importance and relevance of studying the ground squirrel in as a natural model for the avoidance of skeletal muscle wasting and cardiac hypertrophy (Cotton \& Harlow, 2015; Gao et al., 2012; Li et al., 2013; Nelson \& Rourke, 2013; Xu et al., 2013). Specifically, recent findings indicated that PGC-1 $\alpha$ and the process of mitochondrial biogenesis, which is usually active during exercise, is active as well during hibernation and may be contributing to the preservation of muscle mass and fiber type (Xu et al., 2013). Therefore, by improving our understanding of how ground squirrels adjust their cellular processes, specifically transcriptional regulation, in response to changes in $\mathrm{T}_{\mathrm{b}}$ and nutritional stresses during hibernation, we gain an increased understanding of the molecular mechanisms of natural muscle remodeling.

The work presented in this thesis highlights three themes in hibernation biochemistry and molecular biology: 1) adaptations of transcription factor regulation, and 2) muscle remodeling regulation through alterations of structural and ubiquitin ligase proteins in skeletal and cardiac 
muscle, as well as 3) the impact of environmental factors like temperature and $\mathrm{Ca}^{2+}$ on transcription factor binding affinity. During torpor, squirrels conserve energy in all tissues by reducing the majority of metabolic functions. This metabolic rate depression allows squirrels to save up to $88 \%$ of active ATP expenditure, and transcription as well as translation are processes that are suppressed to save energy (Wang \& Lee, 2011). However, as demonstrated in this thesis, the expression of certain genes need to be maintained if not elevated during torpor in order to reduce cellular and tissue damage. Typically, unloading of skeletal muscle results in the activation of catabolic, protein degradation networks like the ubiquitin proteasome system (UPS) and inhibition of anabolic pathways like the insulin-mTOR pathway (Bassel-Duby \& Olson, 2006; Choi et al., 2009; Glass, 2010; Malatesta et al., 2009; Rourke et al., 2004). Conversely, cardiac muscle hypertrophy is characterized by enhanced protein synthesis and protein degradation; resulting in the protein turnover necessary for cardiac remodeling to occur. Both of these mechanisms are activated by increased cardiac workload due to pressure or volume (Depre et al., 2006). These changes that occur due to the unloading of skeletal muscle and the loading of cardiac muscle may be beneficial to meet the bodily demands of nonhibernating mammals at first, however these mammals (i.e. humans) lack the adaptive capabilities to reverse these processes, as a result significant skeletal muscle wasting and heart failure may occur (Bricceno et al., 2012; Day, 2013; Frey et al., 2004; Haslett et al., 2003). This suggests that the hibernator skeletal and cardiac muscle tissues possess unique and beneficial molecular mechanisms that contribute to their survival and preservation.

\section{Regulation of the NFAT-calcineurin pathway in skeletal muscle}


This thesis has identified the roles and regulation of the NFAT-calcineurin pathway and provided evidence of the importance of this pathway to the preservation and maintenance of skeletal muscle during hibernation. NFATc2 protein levels were elevated during torpor along with the DNA-binding activity of NFATc1, c3, and $\mathrm{c} 4$. The expression pattern suggests that NFAT TFs appear to be positively regulated during torpor as a result of the upregulation in calcineurin and $\mathrm{Ca}^{2+}$-signaling proteins like CAM and calpain1, which activate the NFATcalcineurin pathway. The activation of the NFAT-calcineurin pathway during torpor led to the positive regulation of skeletal muscle gene transcription, specifically of targets like myoferlin that contribute to skeletal muscle growth (Demonbreun et al., 2010; Doherty et al., 2005).. Further experiments conducted to study the effect of the cellular environment on NFAT-DNA binding affinity showed that nuclear $\mathrm{Ca}^{2+}$ is actually an inhibitor of NFATc1-binding to DNA. What was even more fascinating was that with decreases in temperature, like those observed with the transition from active and arousal stages $\left(37^{\circ} \mathrm{C}\right)$ to torpor $\left(4^{\circ} \mathrm{C}\right)$ during hibernation, there is a significant decrease in NFAT-DNA binding affinity. Furthermore, the inhibitory effect of temperature on transcription factor binding differentially affects NFAT TFs, where NFATc3binding was not decreased at low temperatures to the extent that NFATc1 and c4 were.

These findings provide important insight into the molecular mechanisms that are responsible for the preservation and/or change of muscle structure/function during hibernation, as they are not well known aside from the finding indicating that PGC-1 $\alpha$ is upregulated to promote mitochondrial biogenesis, which maintains the ratio of oxidative slow-twitch muscle fibers during torpor (Xu et al., 2013). Therefore, the present study identified that transcriptional regulation by the NFAT TFs, which have been shown to regulate targets associated with skeletal muscle hypertrophy and development, plays an important role in skeletal muscle remodeling 
during torpor (Armand et al., 2008; Delling et al., 2000; Hudson et al., 2014). This is evidenced as the downstream muscle membrane protein associated with muscle development and regeneration, myoferlin, was upregulated. In addition to myoferlin, myomaker is a membrane protein found in the muscle that also controls myoblast fusion and results in the complete loss of myoblast fusion when it is mutated, resulting in the absence of all skeletal muscle, which leads to postnatal death in myomaker-null mice (Millay et al., 2013). In addition, myomaker expression and increased myoblast fusion has been shown to occur in adult satellite cells following muscle injury (Millay et al., 2014). The present thesis showed novel evidence that NFATc1, c3, and c4 could possibly bind to the myomaker promoter, and this novel finding could lead to further studies that characterize NFAT regulation of this vital myogenic protein. These findings implicate both myomaker and myoferlin as novel candidates for muscular dystrophy and Spinal Muscular Atrophy; diseases characterized by significant muscle weakness, degeneration, and atrophy, due to their roles in muscle formation and repair (Lorson, Rindt, \& Shababi, 2010; Marston \& Hodgkinson, 2001).

Calcineurin is a calmodulin-stimulated protein phosphatase that regulates NFATs through dephosphorylation, thereby activating and allowing NFATs to translocate to the nucleus and regulate gene transcription (Rusnak \& Mertz, 2000). CAM is a ubiquitously expressed $\mathrm{Ca}^{2+}-$ binding protein that is involved in a variety of signaling pathways that are $\mathrm{Ca}^{2+}$-dependent. It regulates calcineurin by binding to the regulatory domain of the calcineurin A subunit when it is exposed due to conformational changes caused by activation of the calcineurin B subunit when there is an increase in intracellular $\mathrm{Ca}^{2+}$ levels (Klee et al., 1979; Yang \& Klee, 2000). When calcineurin $\mathrm{B}$ binds to $\mathrm{Ca}^{2+}$ ions, a conformational change occurs in its $\mathrm{C}$-terminal autoinhibitory domain, and the $\mathrm{Ca}^{2+}$-dependent cysteine protease calpain, specifically calpain1/calpain- $\mu$, 
cleaves the autoinhibitory domain, thus activating calcineurin (Burkard, 2005; Lee et al., 2014; Shioda et al., 2006). Therefore, both CAM and calpain1 are important regulators of the NFATcalcineurin pathway. During torpor, the amplitude of $\mathrm{Ca}^{2+}$ transients following excitation is increased following excitation at low temperatures, and as a result, stronger contractions with higher amplitudes are seen at lower temperatures (Liu et al., 1993; Liu et al., 1990; Wang et al., 2000; Wang et al., 1997). Therefore, greater spikes in intracellular $\left[\mathrm{Ca}^{2+}\right]$ following an action potential and the upregulation of the above-mentioned $\mathrm{Ca}^{2+}$ proteins could be causing the increase in NFAT activity, allowing for a maintenance of muscle mass during hibernation.

Given the extreme environmental stressors confronting 13-lined ground squirrels during hibernation, we suspected that environmental factors such as temperature could potentially affect the binding ability of NFATs, and potentially other TF, to DNA. Recent literature has begun to show that gene expression could be affected by temperature, but no study has directly investigated the temperature dependence of TF-binding to DNA (Novák et al., 2015; Chen et al., 2015; Riehle et al., 2003; Swindell et al., 2007). Therefore, our novel finding that NFAT TFs have lower binding affinity to target genes at lower temperatures provides evidence of another mechanism by which hibernators undergo metabolic rate depression during torpor. Due to the need to conserve energy, ground squirrels may enter torpor and decrease their $\mathrm{T}_{\mathrm{b}}$ for the purpose of decreasing transcription through an inhibition of TF binding. However, within the context of studying the roles of different NFATs and their regulation of muscle remodeling, what is even more fascinating is that we found differential regulation of TF binding by temperature. NFATc3 is one of the most important NFATs in terms of regulating the expression of downstream muscle proteins like myoferlin, therefore the decrease in TF binding was reduced for this NFAT in comparison with NFATs c1 and c4 (Delling et al., 2000; Demonbreun et al., 2010). This finding 
implies that ground squirrels downregulate gene expression of genes that play a less important role in survival during torpor compared to genes that play more significant roles. The thermoregulation of TFs certainly warrants further study as will be discussed in the future directions section. However, these novel findings provide important insight into our understanding of TFs and their regulation of gene expression, and finding how transcription factors are thermoregulated as well as its regulation by other environmental stimuli, could lead to tissue- and cell-specific targeting of therapeutics that activate or inhibit transcription factors.

\section{Regulation of the NFAT-calcineurin pathway in cardiac muscle}

Hibernation is also a unique and natural model whereby transitions into hibernation cause cardiomyocyte hypertrophy to occur in a way that is comparable to the way in which human cardiac muscle experiences hypertrophy, which results in fibrosis, hypothermia, and eventually heart failure (Frey et al., 2004; Nelson \& Rourke, 2013). This thesis has elucidated a mechanism by which the NFAT TFs regulate cardiac hypertrophy during torpor, and the reversal of this process during arousal. Importantly, NFATs $\mathrm{c} 2$ and $\mathrm{c} 3$ were both elevated during ET, and as a result, myoferlin and myomaker protein levels were significantly elevated at this time point as well. Therefore, we suspect that the end of the decline in $\mathrm{T}_{\mathrm{b}}$ to $4^{\circ} \mathrm{C}$ during torpor, which occurs at ET, may be an important initiator for cardiac hypertrophy to occur in the hearts of these animals. We also suspect that this upregulation of NFATc2 and c3, along with the initiation of cardiac hypertrophy, may be regulated in part by $\mathrm{Ca}^{2+}$ signaling through the progressive increase in calcineurin protein levels during torpor and the rise in CAM levels at EN. 
$\mathrm{Ca}^{2+}$ signaling, involving the two above-mentioned proteins in addition to calpain, appear to play an even more significant role in the reversal of cardiac hypertrophy as the squirrel becomes aroused. The expression of NFATc3, myoferlin, and myomaker declines following the sharp increase at ET, and NFATc2 levels continue to progressively decline following ET and into arousal. The decline in the expression of myoferlin and myomaker appear to be regulated by the $\mathrm{Ca}^{2+}$ signaling proteins, especially CAM and calpain-1, whose protein levels both decline significantly throughout the torpor-arousal cycle despite the stable levels of calcineurin. As mentioned previously, CAM and calpain both activate calcineurin and the NFAT-calcineurin pathway through independent mechanisms (Burkard, 2005; Klee et al., 1979; Lee et al., 2014; Shioda et al., 2006; Yang \& Klee, 2000). Therefore, decreased levels of these activators following torpor could be causing the decline in NFATc2, c3, myoferlin, and myomaker protein levels, thus resulting in attenuation if not reversal of cardiac hypertrophy. This pattern of expression for CAM and calpain in cardiac muscle is in sharp contrast to what was observed in the skeletal muscle, where these proteins were highly expressed during torpor. This is one example of the specificity by which gene expression is controlled during hibernation in a tissuespecific manner.

Furthermore, myomaker, a protein vital to muscle development and regeneration from injury, was upregulated during torpor in cardiac muscle but not skeletal muscle (Millay et al., 2013). The regulation of myomaker may depend on the severity of injury induced on the muscle, since it was up-regulated following cardiotoxin-induced injury. Cardiotoxin is a myotoxic agent that leads to the myolysis of the myofiber by inducing rapid plasma membrane depolarization and the characteristics of muscle regeneration depend on the type of injury induced (Czerwinska, Streminska, Ciemerych, \& Grabowska, 2012). Therefore, the levels of skeletal muscle atrophy 
that the ground squirrel goes through during hibernation may not be enough to induce an increase in myomaker. However, the stress induced upon the cardiac muscle during hibernation did present a great enough stress to induce an increase in myomaker expression. In order for cardiomyocytes to experience a reduction in size, significant atrophy and protein degradation needs to occur, therefore we suspect that the UPS is activated as well as the squirrels are being aroused from torpor, and these findings will be discussed in a subsequent section (Depre et al., 2006; Galasso et al., 2010; Herrmann et al., 2007).

In summary, our findings on the importance of $\mathrm{Ca}^{2+}$-signaling in mediating activation of the NFAT-calcineurin pathway during torpor to increase the expression of important targets like myoferlin and myomaker that promote cardiac hypertrophy during torpor furthers our knowledge of cardiac muscle remodeling. Furthermore, the finding that decreased expression of CAM and calpain initiated an inhibition of NFATc2 and c3 as well as decreased expression of myoferlin and myomaker implicates these targets in therapeutic intervention with the hopes of reversing cardiac hypertrophy and preventing heart failure. The testing and development of inhibitors for the Calcineurin-NFAT pathway is needed to observe for a reversal in the hypertrophic response. The unique and tissue-specific mechanisms in ground squirrel of preserving skeletal muscle and undergoing reversible cardiac hypertrophy during the torpor-arousal cycle make studying this animal biologically and clinically-relevant.

\section{Adaptations of muscle atrophy regulation in skeletal muscle}

During hibernation, one would expect that muscle atrophy would occur due to mechanical unloading, where muscles are activated less frequently and for shorter periods especially during torpor than when squirrels are active. This would result in continual 
deterioration of skeletal muscle as this mechanical unloading stimulates protein degradation pathways like the UPS (Herrmann et al., 2007; Schiaffino et al., 2013). However, previous work has suggested that the ground squirrel is able to avoid significant muscle wasting during hibernation despite the prolonged periods of mechanical unloading, especially during torpor (Cotton \& Harlow, 2015; Gao et al., 2012; Xu et al., 2013). However, there have been no studies that have been conducted to investigate how the UPS and other mechanisms of protein degradation are regulated during hibernation. Findings from previous studies as well as this thesis have shown that regulators of skeletal muscle hypertrophy and remodeling, like the NFAT-calcineurin pathway and PGC- $1 \alpha$ regulation of mitochondrial biogenesis in muscle, are activated during torpor. However, there is a current gap in knowledge regarding whether pathways regulating muscle atrophy are activated during torpor as a result of inactivity, but hypertrophic pathways like the NFAT-calcineurin pathway simply balance atrophy in order to preserve muscle, or if muscle atrophy is downregulated during torpor, thus contributing to the avoidance of muscle wasting.

The UPS mechanism for protein degradation occurs via ubiquitin ligases, like MAFbx and MURF1, which ligate substrates to ubiquitin in order to target these substrates for degradation. MyoG and Foxo4 are two important regulators of the UPS through their transcriptional regulation of both MAFbx and MURF1 (Moresi et al., 2010; Sandri et al., 2004; Stitt et al., 2004). Our findings indicated that Foxo4 protein levels were significantly upregulated during torpor, whereas MyoG levels were decreased. Furthermore, similarly to MyoG, MAFbx and MURF1 expression were decreased as well during torpor. Initially, this finding could be interpreted as evidence that there is differential regulation of MAFbx and MURF1 by Foxo4 and MyoG, and it is surprising that the Foxo4 expression pattern contradict those of MAFbx and 
MURF1. However, Foxo4 activation (defined by its ability to regulate transcription) as well as its nuclear localization were studied by analyzing the levels of specific phosphorylated residues of Foxo4. Akt/PKB inhibits the nuclear translocation and activation of Foxo4 by phosphorylating the Ser-197 residue on Foxo4 (Matsuzaki et al., 2005; Takaishi et al., 1999). Our results indicate the p-Foxo4 S197 levels decreased significantly throughout the torpor, suggesting that there is an increase in Foxo4 nuclear translocation. Alternatively, the Ras-Ral pathway regulates Foxo4 transcriptional activity through the phosphorylation of Foxo 4 at Thr- 451 by the downstream kinase, JNK1 (De Ruiter et al., 2001; Kops et al., 1999; Van Den Berg et al., 2013). This mechanism is independently from the control of nuclear-cytoplasmic distribution that Akt holds over Foxo4. p-Foxo4 T451 levels as well as the ratios of p-Foxo4/total Foxo4 both decreased significantly during torpor, suggesting that although Foxo4 is upregulated and is able to translocate to the nucleus, it is not transcriptionally active. Furthermore, this inactivation of Foxo4 was shown to be regulated by the Ras-Ral pathway involving the Ras, RalA, and Ralbp1 proteins that are all downregulated during torpor.

These findings suggest that in addition to upregulating pathways that moderate and promote hypertrophy and fiber type switching towards slow, oxidative fibers, I. tridecemlineatus also downregulates pathways that result in protein degradation and muscle atrophy. This later process is initiated by inhibition of the Ras-Ral pathway, as shown by decreased Ras, RalA, and Ralbp1 protein levels during torpor, thus leading to inactivation of Foxo4 during torpor. This, along with downregulation of MyoG, results in the decreased expression of MAFbx and MURF1 to decrease protein degradation through the UPS. These novel findings on this unique and natural physiological process in ground squirrels advances our knowledge of skeletal muscle remodeling, and they could be applied for therapeutic intervention in treating muscle wasting diseases like Spinal 
Muscular Atrophy and Duchenne Muscular Dystrophy. Specifically, our findings suggest that in addition to testing agents that promote muscle growth, like Naloxone - an NFAT activator, there needs to be an emphasis on testing inhibitors of muscle atrophy signaling pathways. For example, farnesyltransferase inhibitors designed to block the Ras-Ral pathway for anticancer treatment could also be tested for the possibility of inhibiting Foxo4 and reducing muscle atrophy (Yeh \& Der, 2007).

\section{Adaptations of muscle atrophy regulation in cardiac muscle}

As demonstrated previously in this thesis, upregulation of the NFAT-calcineurin pathway has been implicated in the increased synthesis of numerous proteins during cardiac hypertrophy. With regard to protein degradation, the UPS is suspected to be an important mechanism whereby substrates are ligated to ubiquitin via ubiquitin ligases and are targeted for degradation (Herrmann et al., 2007). The specificity of the UPS is determined by E3 ubiquitin ligases, such as MAFbx and MURF1, which recognize specific target proteins. The role of the UPS remains relatively unclear in cardiac muscle in comparison with skeletal muscle; although they have shown upregulation in association with cardiac hypertrophy or heart failure (Depre et al., 2006; Galasso et al., 2010). This increased in expression of ubiquitination machinery may be in response to the increase in over protein production that accompanies hypertrophy in the heart or in response to modified or damaged proteins that need to be degraded (Day, 2013). Therefore, with respect to reversible cardiac hypertrophy, we suspect that the UPS will be mostly active during late torpor or arousal, especially when coming out of hibernation as perfusion and $\mathrm{T}_{\mathrm{b}}$ will increase; thus reversing the hypertrophic stimulus; promoting atrophy instead.

The Foxo TFs; Foxo1, 3a, 4, as well as MyoG all regulate the expression of MAFbx and MURF1 (Moresi et al., 2010; Sandri et al., 2004; Stitt et al., 2004). Furthermore, activation of these Foxo TFs rely upon Akt/PKB as it has been shown to block the function of all three Foxo 
proteins through phosphorylation, leading to their containment in the cytoplasm (Brunet et al., 1999; Takaishi et al., 1999; Tang et al., 1999). Akt has numerous phosphorylation sites on Foxo1, 3a, and 4, including Threonine ${ }^{32}\left(\mathrm{Thr}^{32}\right)$ for Foxo3a, as well as $\mathrm{Thr}^{24}$ and Serine $\mathrm{e}^{319}$ $\left(\mathrm{Ser}^{319}\right)$ for Foxo1 (Dobson et al., 2011). Our findings confirmed our hypothesis, as we found that there is upregulation of Foxo4 and MyoG during LT. MAFbx and MURF1 followed the same pattern of expression, where they increased in late torpor as well as arousal, thus suggesting that the UPS is activated as ground squirrel are aroused from torpor, which could be causing muscle atrophy and the reversal of cardiac hypertrophy.

On the other hand, we also identified increases Foxo1 and 3a protein levels as well as decreases in inactive, phosphorylated Foxo1 and 3a proteins during torpor in comparison with euthermic control, suggesting these Foxos are actually significantly activated during torpor. Foxo1 and 3a are other factors believed to regulate the expression of MAFbx and MURF1 but they have also been implicated in a vast number of cellular processes in addition to protein degradation; these processes include cell cycle inhibition, anti-oxidative response, and a shift in cellular metabolism away from anabolic processes towards catabolic metabolism (Eijkelenboom \& Burgering, 2013; Tessier \& Storey, 2016; van der Horst \& Burgering, 2007; Wu \& Storey, 2014). Therefore, the present results demonstrate that the signaling pathway involving Foxo4, MyoG, and the E3 ligases MAFbx and MURF1 play a significant role in cardiac muscle remodeling in squirrels, which provide the molecular basis underlying the reversal of cardiac hypertrophy, a process unique to hibernators.

\section{Future Directions}


This thesis is the first to identify changes in NFAT-DNA binding affinity that are temperature-dependent, although further studies need to be conducted to determine whether our findings are specific for NFAT TFs or if it reflects a greater number of TFs. More importantly, further studies need to determine whether the temperature-sensitivity of NFAT transcription factors are due to conformational changes that occur at lower temperatures to the protein itself, to DNA, or if it has to do with interactions with temperature-sensitive cofactors. For example, NFATc2 has been shown to cooperate with heat shock transcription factor 1 (HSF1), which is responsible for regulating the gene expression of other heat shock proteins (Hayashida et al., 2010). Recent literature has begun to show that gene expression could be affected by temperature, but no study has directly investigated the temperature dependence of transcription factor-binding to DNA (Novák et al., 2015; Chen et al., 2015; Riehle et al., 2003; Swindell et al., 2007). Most of these studies use DNA microarrays to study the global changes in gene expression when temperature stress is induced on an organism (Riehle et al., 2003; Swindell et al., 2007). However, although this approach identifies targets that may be involved in stressresponse, it does not directly elucidate mechanisms such as transcription factor binding affinity.

In addition to the ground squirrel's ability to thermoregulate during torpor-arousal cycles, they also show enhanced capabilities to maintaining intracellular $\mathrm{Ca}^{2+}$ and urea concentrations in comparison with non-hibernating animals under the same temperature stress (Chilian \& Tollefson, 1976; Kristofferson, 1963; Liu et al., 1991; Wang \& Zhou, 1999; Wang et al., 1999; Wang et al., 2002). It was observed in this thesis that progressively increasing $\left[\mathrm{Ca}^{2+}\right]$ decreased the binding of NFATc1, whereas NFATc4 showed moderately increased binding to DNA when $\left[\mathrm{Ca}^{2+}\right]$ was increased to $600 \mathrm{nM}$. These effects of intranuclear $\mathrm{Ca}^{2+}$ on NFAT-DNA binding during torpor seem to be specific for each NFAT transcription factor, therefore this effect is 
likely not due to the binding and blocking of DNA by intranuclear $\mathrm{Ca}^{2+}$ (Dobi \& Agoston, 1998). This effect is most likely due to $\mathrm{Ca}^{2+}$ regulation of specific export kinases like CAMKIV or through specific coactivators of individual NFATs, such as CBP (Chawla et al., 1998; Yang et al., 2001). However, more research needs to be conducted to further elucidate the mechanism behind how nuclear $\mathrm{Ca}^{2+}$ regulates TF-binding to DNA.

Myoferlin and myomaker are proteins that are well-studied with regard to their importance and contribution towards myoblast fusion, muscle hypertrophy, regeneration, and repair (Demonbreun et al., 2010; Doherty et al., 2005; Millay et al., 2013, 2014). However, their function in cardiac muscle is more of a mystery. Myoferlin was known to be highly expressed in skeletal muscle and to a lesser degree in cardiac muscle, and we confirmed that it is expressed in cardiac muscle in a pattern that is correlated with cardiac hypertrophy (Davis et al., 2000; Zhang \& Storey, 2015). Myomaker on the other hand, has only recently been discovered and its expression has not been identified in any tissue type other than skeletal muscle. Therefore, the current study was the first to identify the expression of myomaker in cardiac muscle. However, its role as well as the role of myoferlin in cardiac hypertrophy and remodeling needs to be studied further as it is known that cardiac hypertrophy and skeletal muscle hypertrophy occur via very different mechanisms. In skeletal muscle, individual myoblast cells proliferate and differentiate into fully mature muscle cells called myotubes, which form muscle fibers (Bentzinger, Wang, \& Rudnicki, 2012). However, in cardiac muscle, hypertrophy occurs via an increase in the size of cardiomyocytes, and there is heightened organization of the sarcomere but very little proliferation of cardiomyocytes occurs (Hill \& Olson, 2008). Therefore, it is very important to study the exact function of myoferlin and myomaker within cardiomyocytes using knock-out models. Furthermore, myoferlin has been studied recently in relation to cancer in 
multiple tissues, so this important structural protein as well as myomaker should be studied in cell types other than muscle (Bernatchez, Sharma, Kodaman, \& Sessa, 2009; Leung, Yu, Lin, Tognon, \& Bernatchez, 2013; Turtoi et al., 2013; Yu et al., 2011). Furthermore, Myomaker is also known as Transmembrane protein 8c (TMEM8C), however there are two other members of the family. Therefore TMEM8a and $8 b$ should be further studied for their potential function or lack thereof in the context of skeletal muscle and cardiac muscle remodeling.

Given the muscle remodeling that occurs over the course of the torpor-arousal cycles in both skeletal and cardiac muscle, it is expected that nitrogen balance will be affected as a result of the hypertrophy and protein synthesis occurring during torpor in both tissues and the protein degradation that occurs in cardiac muscle during arousal. By measuring tissue nitrogen isotope ratios, Lee et al. (2012) observed no changes in skeletal muscle but an increase in the isotope ratios in heart during hibernation. This suggests that in the skeletal muscle, there is a balance between protein breakdown and synthesis, which supports the idea that squirrels are able to maintain and preserve muscle mass during hibernation. On the other hand, in the heart, the increase in the nitrogen isotope ratio indicates that there is greater protein synthesis than breakdown occurring in this organ (Lee et al., 2012). This also supports the idea that cardiac hypertrophy occurs during hibernation. However, the study of nitrogen balance needs to be extended to timecourse experiments in order to understand how nitrogen balance is affected by the constantly-changing molecular stimuli for protein synthesis and degradation that occurs over the torpor-arousal cycle.

In summary, this thesis studied the molecular response of striated muscles to the natural process of hibernation, which is characterized by metabolic rate depression, cold body 
temperatures, and drastic physiological changes. The analysis of the NFAT transcription factors, along with their regulation through calcium signaling, and their downstream targets (myoferlin, myomaker), has shown the importance of the NFAT-calcineurin pathway in ensuring the survival of the organism and in the avoidance of disuse-induced muscle atrophy as well as in regulating reversible cardiac hypertrophy. Furthermore the regulation of NFAT TFs by environmental stimuli such as temperature and intranuclear $\left[\mathrm{Ca}^{2+}\right]$ suggested that these TFs as well as others may be regulated by both conditions. The analysis of the Foxo family of TFs, its regulation through the Akt and Ras-Ral pathways, the MyoG TF, in addition to the ubiquitin ligases MAFbx and MURF1, indicated that downregulation of muscle atrophy in skeletal muscle during torpor and in cardiac muscle during arousal contribute to the avoidance of skeletal muscle loss and in reversing cardiac hypertrophy during and after hibernation, respectively. Clearly the process of hypometabolism in the thirteen-lined ground squirrel is a complex process that is highly regulated. However, by comparing the effective physiological adaptations in this system during hibernation to humans provides insight into how we can combat certain diseases and live efficiently like squirrels. 


\section{Publication List}




\section{Research Articles Accepted}

Yichi Zhang, Shannon N. Tessier, Kenneth B. Storey. 2016. Inhibition of skeletal muscle atrophy during torpor in ground squirrels occurs through downregulation of MyoG and inactivation of Foxo4. Cryobiology. Accepted.

Yichi Zhang, Oscar Aguilar, Kenneth B. Storey. 2016. Transcriptional activation of muscle atrophy promotes cardiac muscle remodeling during mammalian hibernation. PeerJ, 4, e2317. DOI:10.7717/peerj.2317.

Yichi Zhang, Kenneth B. Storey. 2016. Regulation of gene expression by NFAT transcription factors in hibernating ground squirrels is dependent on the cellular environment. Cell Stress and Chaperones, 21, 883-894. DOI:10.1007/s12192-016-0713-5.

Yichi Zhang, Kenneth B. Storey. 2015. Expression of nuclear factor of activated T cells (NFAT) and downstream muscle-specific proteins in ground squirrel skeletal and heart muscle during hibernation. Molecular Cell Biochemistry, 412, 27-40. DOI:10.1007/s11010-0152605-x.

\section{Research Articles in Review}

Rasha Al-attar, Yichi Zhang, Kenneth B. Storey. Osmolyte regulation by TonEBP/NFAT5 during anoxia-recovery and dehydration-rehydration stresses in the freeze-tolerant wood frog (Rana sylvatica). PeerJ. In Review.

\section{Conference Papers}


Zhang Y, Storey KB (2015) Expression of nuclear factor of activated T cells (NFAT) and downstream muscle-specific proteins in ground squirrel cardiac muscle. Canadian Journal of Cardiology, 31(10), S143. doi:10.1016/j.cjca.2015.07.311

\section{Other Peer-Reviewed Publications}

Zhang Y (2015) Epigenomics: biological and clinical implications of histone deacetylation. Health Science Inquiry, 6.

\section{Communications and Scientific Meetings}

\section{Poster Presentations}

Zhang Y, Tessier SN, Storey KB. Expression of nuclear factor of activated T-cells (NFAT) and downstream muscle-specific proteins in ground squirrel skeletal and heart muscle during hibernation, Cryobiology 2016 Conference, Ottawa, Ontario. July, 2016.

Zhang Y, Aguilar AA, Storey KB. Transcriptional activation of muscle atrophy promotes cardiac muscle remodelling during mammalian hibernation, Ottawa-Carleton Institute of Biology Conference, Ottawa, Ontario. April, 2016.

Zhang Y, Aguilar AA, Storey KB. Transcriptional activation of muscle atrophy promotes cardiac muscle remodelling during mammalian hibernation. $18^{\text {th }}$ Annual Chemistry and Biochemistry Graduate Research Conference, Montreal, Quebec. November, 2015.

Zhang Y, Storey KB. Expression of nuclear factor of activated T cells (NFAT) and downstream muscle proteins in ground squirrel cardiac muscle. Canadian Cardiovascular Congress, Toronto, Ontario. October, 2015. 
Zhang Y, Storey KB. Expression of nuclear factor of activated T cells (NFAT) and downstream targets in ground squirrel skeletal muscle during hibernation. 21 ${ }^{\text {st }}$ Annual Canadian Connective Tissue Conference, Quebec City, Quebec. May, 2015.

Zhang Y, Storey KB. Expression of the Nuclear Factor of Activated T cells and downstream targets in ground squirrel cardiac muscle, Ottawa-Carleton Institute of Biology Conference, Ottawa, Ontario. April, 2015.

Zhang Y, Storey KB. Expression of the Nuclear Factor of Activated T cells and muscle-specific targets in ground squirrel cardiac muscle, Ottawa Heart Research Conference, Ottawa, Ontario. April, 2015.

\section{Oral Presentations}

Expression of nuclear factor of activated T cells (NFAT) and downstream targets in ground squirrel skeletal muscle during hibernation, Ottawa-Carleton Institute of Biology Conference, Ottawa, Ontario (Oral). 


\section{References}


Al-Shanti, N., \& Stewart, C. E. (2009). Ca2+/calmodulin-dependent transcriptional pathways: potential mediators of skeletal muscle growth and development. Biological Reviews, 84, 637-652. http://doi.org/10.1111/j.1469-185X.2009.00090.x

Armand, A-S., Bourajjaj, M., Martínez-Martínez, S., el Azzouzi, H., da Costa Martins, P. a, Hatzis, P., ... De Windt, L. J. (2008). Cooperative synergy between NFAT and MyoD regulates myogenin expression and myogenesis. The Journal of Biological Chemistry, 283(43), 29004-29010. http://doi.org/10.1074/jbc.M801297200

Bassel-Duby, R., \& Olson, E. N. (2006). Signaling pathways in skeletal muscle remodeling. Annual Review of Biochemistry, 75, 19-37. http://doi.org/10.1146/annurev.biochem.75.103004.142622

Bentzinger, C. F., Wang, Y. X., \& Rudnicki, M. A. (2012). Building muscle: molecular regulation of myogenesis. Cold Spring Harbor Perspectives in Biology, 4(2), 1-16. http://doi.org/10.1101/cshperspect.a008342

Bernatchez, P. N., Sharma, A., Kodaman, P., \& Sessa, W. C. (2009). Myoferlin is critical for endocytosis in endothelial cells. American Journal of Physiology. Cell Physiology, 297, C484-C492. http://doi.org/10.1152/ajpcell.00498.2008

Birkenkamp, K. U., \& Coffer, P. J. (2003). Regulation of cell survival and proliferation by the FOXO (Forkhead box, class O) subfamily of Forkhead transcription factors. Biochemical Society Transactions, 31(Pt 1), 292-297. http://doi.org/10.1042/BST0310292

Boyer, J. G., Deguise, M-O., Murray, L. M., Yazdani, A., De Repentigny, Y., BoudreauLarivière, C., \& Kothary, R. (2014). Myogenic program dysregulation is contributory to disease pathogenesis in spinal muscular atrophy. Human Molecular Genetics, 23(16), 4249- 
4259. http://doi.org/10.1093/hmg/ddu142

Brand, L. H., Henneges, C., Schüssler, A., Kolukisaoglu, H. Ü., Koch, G., Wallmeroth, N., ... Wanke, D. (2013). Screening for Protein-DNA Interactions by Automatable DNA-Protein Interaction ELISA. PLoS ONE, 8(10), e75177. http://doi.org/10.1371/journal.pone.0075177

Brand, L. H., Kirchler, T., Hummel, S., Chaban, C., \& Wanke, D. (2010). DPI-ELISA: a fast and versatile method to specify the binding of plant transcription factors to DNA in vitro. Plant Methods, 6(1), 25. http://doi.org/10.1186/1746-4811-6-25

Bricceno, K. V., Sampognaro, P. J., Van meerbeke, J. P., Sumner, C. J., Fischbeck, K. H., \& Burnett, B. G. (2012). Histone deacetylase inhibition suppresses myogenin-dependent atrogene activation in spinal muscular atrophy mice. Human Molecular Genetics, 21(20), 4448-4459. http://doi.org/10.1093/hmg/dds286

Brière, C., Xiong, T. C., Mazars, C., \& Ranjeva, R. (2006). Autonomous regulation of free Ca2+ concentrations in isolated plant cell nuclei: A mathematical analysis. Cell Calcium, 39(4), 293-303. http://doi.org/10.1016/j.ceca.2005.11.005

Brown, J. C. L., Chung, D. J., Belgrave, K. R., \& Staples, J. F. (2012). Mitochondrial metabolic suppression and reactive oxygen species production in liver and skeletal muscle of hibernating thirteen-lined ground squirrels. American Journal of Physiology. Regulatory, Integrative and Comparative Physiology, 302(1), R15-28. http://doi.org/10.1152/ajpregu.00230.2011

Brunet, A., Bonni, A., Zigmond, M. J., Lin, M. Z., Juo, P., Hu, L. S., ... Greenberg, M. E. (1999). Akt promotes cell survival by phosphorylating and inhibiting a Forkhead transcription factor. Cell, 96(6), 857-868. http://doi.org/10.1016/S0092-8674(00)80595-4 
Buck, C. L., \& Barnes, B. M. (2000). Effects of ambient temperature on metabolic rate, respiratory quotient, and torpor in an arctic hibernator. American Journal of Physiology. Regulatory, Integrative and Comparative Physiology, 279(1), R255-R262.

Eijkelenboom, A., \& Burgering, B. M. T. (2013). FOXOs: signalling integrators for homeostasis maintenance. Nature Reviews. Molecular Cell Biology, 14(2), 83-97. http://doi.org/10.1038/nrm3507

Burkard, N. (2005). Targeted Proteolysis Sustains Calcineurin Activation. Circulation, 111(8), 1045-1053. http://doi.org/10.1161/01.CIR.0000156458.80515.F7

Cantor, S. B., Urano, T., \& Feig, L. A. (1995). Identification and characterization of Ral-binding protein 1, a potential downstream target of Ral GTPases. Molecular and Cellular Biology, 15(8), 4578-4584. http://doi.org/PMID: 7623849

Chawla, S., Hardingham, G. E., Quinn, D. R., \& Bading, H. (1998). CBP: a signal-regulated transcriptional coactivator controlled by nuclear calcium and CaM kinase IV. Science (New York, N.Y.), 281(5382), 1505-1509. http://doi.org/10.1126/science.281.5382.1505

Chen, J., Nolte, V., \& Schlötterer, C. (2015). Temperature related reaction norms of gene expression: regulatory architecture and functional implications. Molecular Biology and Evolution, 32(9), 1-19. http://doi.org/10.1093/molbev/msv120

Chilian, W. M., \& Tollefson, D. (1976). Blood urea levels and erythrocyte fragility to isosmotic urea during hibernation and activity of Spermophilus tridecimlineatus. Comparative Biochemistry and Physiology -- Part A: Physiology, 54(4), 433-435.

http://doi.org/10.1016/0300-9629(76)90045-1

Cho, Y. Y., Yao, K., Bode, A. M., Bergen, H. R., Madden, B. J., Oh, S. M., ... Dong, Z. (2007). 
RSK2 mediates muscle cell differentiation through regulation of NFAT3. Journal of Biological Chemistry, 282, 8380-8392. http://doi.org/10.1074/jbc.M611322200

Choi, H., Selpides, P.-J. I., Nowell, M. M., \& Rourke, B. C. (2009). Functional overload in ground squirrel plantaris muscle fails to induce myosin isoform shifts. American Journal of Physiology. Regulatory, Integrative and Comparative Physiology, 297, R578-R586. http://doi.org/10.1152/ajpregu.00236.2009

Choi, W., Swanson, S. J., \& Gilroy, S. (2011). Coding and Decoding of Calcium Signals in Plants. Coding and Decoding of Calcium Signals in Plants, 41-61. http://doi.org/10.1007/978-3-642-20829-4

Cotton, C. J., \& Harlow, H. J. (2015). Avoidance of skeletal muscle atrophy in spontaneous and facultative hibernators. Physiological and Biochemical Zoology : PBZ, 83(3), 551-560. http://doi.org/10.1086/650471

Czerwinska, A. M., Streminska, W., Ciemerych, M. A., \& Grabowska, I. (2012). Mouse gastrocnemius muscle regeneration after mechanical or cardiotoxin injury. Folia Histochemica et Cytobiologica, 50(1), 144-153. http://doi.org/10.5603/FHC.2012.0021

Davis, D. B., Delmonte, A. J., Ly, C. T., \& McNally, E. M. (2000). Myoferlin, a candidate gene and potential modifier of muscular dystrophy. Human Molecular Genetics, 9(2), 217-226. http://doi.org/ddd030 [pii]

Davis, D. B., Doherty, K. R., Delmonte, A. J., \& McNally, E. M. (2002). Calcium-sensitive phospholipid binding properties of normal and mutant ferlin C2 domains. Journal of Biological Chemistry, 277, 22883-22888. http://doi.org/10.1074/jbc.M201858200 
Day, S. M. (2013). The ubiquitin proteasome system in human cardiomyopathies and heart failure. American Journal of Physiology. Heart and Circulatory Physiology, 304(100), H1283-1293. http://doi.org/10.1152/ajpheart.00249.2012

De Ruiter, N. D., Burgering, B. M., \& Bos, J. L. (2001). Regulation of the Forkhead transcription factor AFX by Ral-dependent phosphorylation of threonines 447 and 451. Molecular and Cellular Biology, 21(23), 8225-8235. http://doi.org/10.1128/MCB.21.23.8225-8235.2001

Delling, U., Tureckova, J., Lim, H. A. E. W., Windt, L. J. D. E., Rotwein, P., \& Molkentin, J. D. (2000). A Calcineurin-NFATc3-Dependent Pathway Regulates Skeletal Muscle Differentiation and Slow Myosin Heavy-Chain Expression. Molecular and Cellular Biology, 20(17), 6600-6611.

Demonbreun, A. R., Lapidos, K. A., Heretis, K., Levin, S., Dale, R., Pytel, P., ... McNally, E. M. (2010). Myoferlin regulation by NFAT in muscle injury, regeneration and repair. Journal of Cell Science, 123(Pt 14), 2413-2422. http://doi.org/10.1242/jcs.065375

Depre, C., Wang, Q., Yan, L., Hedhli, N., Peter, P., Chen, L., .. Madura, K. (2006). Activation of the cardiac proteasome during pressure overload promotes ventricular hypertrophy. Circulation, 114(17), 1821-1828. http://doi.org/10.1161/CIRCULATIONAHA.106.637827

Dobi, A., \& Agoston, D. V. (1998). Submillimolar levels of calcium regulates DNA structure at the dinucleotide repeat (TG/AC)n. Proceedings of the National Academy of Sciences of the United States of America, 95(11), 5981-5986. http://doi.org/10.1073/pnas.95.11.5981

Dobson, M., Ramakrishnan, G., Ma, S., Kaplun, L., Balan, V., Fridman, R., \& Tzivion, G. (2011). Bimodal regulation of FoxO3 by AKT and 14-3-3. Biochimica et Biophysica Acta Molecular Cell Research, 1813(8), 1453-1464. 
http://doi.org/10.1016/j.bbamcr.2011.05.001

Doherty, K. R., Cave, A., Davis, D. B., Delmonte, A. J., Posey, A., Earley, J. U., ... McNally, E. M. (2005). Normal myoblast fusion requires myoferlin. Development (Cambridge, England), 132(24), 5565-5575. http://doi.org/10.1242/dev.02155

Eaton, S. L., Roche, S. L., Llavero Hurtado, M., Oldknow, K. J., Farquharson, C., Gillingwater, T. H., \& Wishart, T. M. (2013). Total Protein Analysis as a Reliable Loading Control for Quantitative Fluorescent Western Blotting. PLoS ONE, 8(8), 1-9. http://doi.org/10.1371/journal.pone.0072457

Eijkelenboom, A., \& Burgering, B. M. T. (2013). FOXOs: signalling integrators for homeostasis maintenance. Nature Reviews. Molecular Cell Biology, 14(2), 83-97. http://doi.org/10.1038/nrm3507

Epperson, L. E., Karimpour-Fard, A., Hunter, L. E., Martin, S. L. (2011). Technology Development for Physiological Genomics Metabolic cycles in a circannual hibernator. Physiological Genomics, 80045, 799-807. http://doi.org/10.1152/physiolgenomics.00028.2011.

Essers, M. A. G., Weijzen, S., de Vries-Smits, A. M. M., Saarloos, I., de Ruiter, N. D., Bos, J. L., \& Burgering, B. M. T. (2004). FOXO transcription factor activation by oxidative stress mediated by the small GTPase Ral and JNK. The EMBO Journal, 23(24), 4802-4812. http://doi.org/10.1038/sj.emboj.7600476

Fahlman, A., Storey, J. M., \& Storey, K. B. (2000). Gene up-regulation in heart during mammalian hibernation. Cryobiology, 40, 332-342. http://doi.org/10.1006/cryo.2000.2254 
Feig, L. A., Urano, T., \& Cantor, S. (1996). Evidence for a Ras/Ral signaling cascade. Trends in Biochemical Sciences. http://doi.org/10.1016/S0968-0004(96)10058-X

Foletta, V. C., White, L. J., Larsen, A. E., Léger, B., \& Russell, A. P. (2011). The role and regulation of MAFbx/atrogin-1 and MuRF1 in skeletal muscle atrophy. Pflugers Archiv: European Journal of Physiology, 461, 325-335. http://doi.org/10.1007/s00424-010-0919-9

Frerichs, K. U., \& Hallenbeck, J. M. (1998). Hibernation in ground squirrels induces state and species-specific tolerance to hypoxia and aglycemia: an in vitro study in hippocampal slices. Journal of Cerebral Blood Flow and Metabolism : Official Journal of the International Society of Cerebral Blood Flow and Metabolism, 18, 168-175. http://doi.org/10.1097/00004647-199802000-00007

Frerichs, K. U., Kennedy, C., Sokoloff, L., \& Hallenbeck, J. M. (1994). Local cerebral blood flow during hibernation, a model of natural tolerance to "cerebral ischemia". Journal of Cerebral Blood Flow and Metabolism : Official Journal of the International Society of Cerebral Blood Flow and Metabolism, 14, 193-205. http://doi.org/10.1038/jcbfm.1994.26

Frey, N., Katus, H. A., Olson, E. N., \& Hill, J. A. (2004). Hypertrophy of the Heart: A New Therapeutic Target? Circulation. http://doi.org/10.1161/01.CIR.0000120390.68287.BB

Galasso, G., De Rosa, R., Piscione, F., Iaccarino, G., Vosa, C., Sorriento, D., ... Chiariello, M. (2010). Myocardial expression of FOXO3a-Atrogin-1 pathway in human heart failure. European Journal of Heart Failure, 12(12), 1290-1296. http://doi.org/10.1093/eurjhf/hfq102

Gao, Y. F., Wang, J., Wang, H. P., Feng, B., Dang, K., Wang, Q., \& Hinghofer-Szalkay, H. G. (2012). Skeletal muscle is protected from disuse in hibernating dauria ground squirrels. 
Comparative Biochemistry and Physiology - A Molecular and Integrative Physiology, 161(3), 296-300. http://doi.org/10.1016/j.cbpa.2011.11.009

Gerace, L., \& Burke, B. (1988). Functional Organization of the nuclear envelope. Annual Review Inc., 4, 335-74.

Glass, D. J. (2010). PI3 kinase regulation of skeletal muscle hypertrophy and atrophy. Current Topics in Microbiology and Immunology, 346(1), 267-278. http://doi.org/10.1007/82-201078

Greer, E. L., \& Brunet, A. (2005). FOXO transcription factors at the interface between longevity and tumor suppression. Oncogene, 24(50), 7410-7425. http://doi.org/10.1038/sj.onc. 1209086

Haslett, J. N., Sanoudou, D., Kho, A. T., Han, M., Bennett, R. R., Kohane, I. S., ... Kunkel, L. M. (2003). Gene expression profiling of Duchenne muscular dystrophy skeletal muscle. Neurogenetics, 4, 163-171. http://doi.org/10.1007/s10048-003-0148-x

Hayashida, N., Fujimoto, M., Tan, K., Prakasam, R., Shinkawa, T., Li, L., .. Nakai, A. (2010). Heat shock factor 1 ameliorates proteotoxicity in cooperation with the transcription factor NFAT. The EMBO Journal, 29(20), 3459-3469. http://doi.org/10.1038/emboj.2010.225

Herrmann, J., Lerman, L. O., \& Lerman, A. (2007). Ubiquitin and ubiquitin-like proteins in protein regulation. Circulation Research. http://doi.org/10.1161/01.RES.0000264500.11888.f0

Hill, J. A., \& Olson, E. N. (2008). Cardiac Plasticity, 1370-1380.

Hindle, A. G., Otis, J. P., Epperson, L. E., Hornberger, T. A., Goodman, C. A., Carey, H. V., \& 
Martin, S. L. (2014). Prioritization of skeletal muscle growth for emergence from hibernation. Journal of Experimental Biology, 218, 276-284.

http://doi.org/10.1242/jeb.109512

Horsley, V., Friday, B. B., Matteson, S., Kegley, K. M., Gephart, J., \& Pavlath, G. K. (2001). Regulation of the growth of multinucleated muscle cells by an NFATC2-dependent pathway. Journal of Cell Biology, 153, 329-338. http://doi.org/10.1083/jcb.153.2.329

Huang, H., Regan, K. M., Lou, Z., Chen, J., \& Tindall, D. J. (2006). CDK2-dependent phosphorylation of FOXO1 as an apoptotic response to DNA damage. Science (New York, N.Y.), 314(5797), 294-297. http://doi.org/10.1126/science.1130512

Hudson, M. B., Woodworth-Hobbs, M. E., Zheng, B., Rahnert, J. A., Blount, M. A., Gooch, J. L., ... Price, S. R. (2014). miR-23a is decreased during muscle atrophy by a mechanism that includes calcineurin signaling and exosome-mediated export. American Journal of Physiology. Cell Physiology, 306(404), C551-558.

http://doi.org/10.1152/ajpcell.00266.2013

Hung, H. F., Wang, B. W., Chang, H., \& Shyu, K. G. (2008). The molecular regulation of resistin expression in cultured vascular smooth muscle cells under hypoxia. Journal of Hypertension, 26, 2349-2360. http://doi.org/10.1097/HJH.0b013e328311fa30

Jagelská E, Brázda V, Pospisilová S., Vojtesek, B., \& Palecek, E. (2002). New ELISA technique for analysis of p53 protein/DNA binding properties. Journal of Immunological Methods, 267, 227-235. http://doi.org/10.1016/S0022-1759(02)00182-5

Jullien-Flores, V., Dorseuil, O., Romero, F., Letourneur, F., Saragosti, S., Berger, R., ... Camonis, J. H. (1995). Bridging Ral GTPase to Rho pathways: RLIP76, a Ral effector with 
CDC42/Rac GTPase-activating protein activity. Journal of Biological Chemistry, 270(38), 22473-22477. http://doi.org/10.1074/jbc.270.38.22473

Kalivendi, S. V, Konorev, E. A., Cunningham, S., Vanamala, S. K., Kaji, E. H., Joseph, J., \& Kalyanaraman, B. (2005). Doxorubicin activates nuclear factor of activated T-lymphocytes and Fas ligand transcription: role of mitochondrial reactive oxygen species and calcium. The Biochemical Journal, 389(Pt 2), 527-539. http://doi.org/10.1042/BJ20050285

Klee, C. B., Crouch, T. H., \& Krinks, M. H. (1979). Calcineurin: a calcium- and calmodulinbinding protein of the nervous system. Proceedings of the National Academy of Sciences, 76(12), 6270-6273. http://doi.org/10.1073/pnas.76.12.6270

Kops, G. J., de Ruiter, N. D., Vries-Smits, A. M., Powell, D. R., Bos, J. L., \& Burgering, B. M. (1999). Direct control of the Forkhead transcription factor AFX by protein kinase B. Nature, 398(April), 630-634.

Kress, T. R., Cannell, I. G., Brenkman, A. B., Samans, B., Gaestel, M., Roepman, P., ... Eilers, M. (2011). The MK5/PRAK Kinase and Myc Form a Negative Feedback Loop that Is Disrupted during Colorectal Tumorigenesis. Molecular Cell, 41(4), 445-457. http://doi.org/10.1016/j.molcel.2011.01.023

Kretsinger, R. (1987). Calcium coordination and the calmodulin fold: divergent versus convergent evolution. Cold Spring Harbor Symposia on Quantitative Biology, 52, 499-510.

Kristofferson, R. (1963). Urea-Levels in Blood and Tissues of Hibernating and Non-Hibernating Hedgehogs. Nature, 197(4865), 402-403.

Lecker, S. H. (2003). Ubiquitin-protein ligases in muscle wasting: multiple parallel pathways? 
Current Opinion in Clinical Nutrition and Metabolic Care, 6(3), 271-275.

http://doi.org/10.1097/01.mco.0000068963.34812.e5

Lee, S. H., Choi, J., Kim, H., Lee, D. H., Roh, G. S., Kim, H. J., ... Cho, G. J. (2014). FK506 reduces calpain-regulated calcineurin activity in both the cytoplasm and the nucleus. Anatomy \& Cell Biology, 47, 91-100. http://doi.org/10.5115/acb.2014.47.2.91

Lee, T. N., Buck, C. L., Barnes, B. M., \& O’Brien, D. M. (2012). A test of alternative models for increased tissue nitrogen isotope ratios during fasting in hibernating arctic ground squirrels. The Journal of Experimental Biology, 215(Pt 19), 3354-3361. http://doi.org/10.1242/jeb.068528

Leung, C., Yu, C., Lin, M. I., Tognon, C., \& Bernatchez, P. (2013). Expression of myoferlin in human and murine carcinoma tumors: Role in membrane repair, cell proliferation, and tumorigenesis. American Journal of Pathology, 182(5), 1900-1909. http://doi.org/10.1016/j.ajpath.2013.01.041

Li, H., Liu, T., Chen, W., Jain, M. R., Vatner, D. E., Vatner, S. F., ... Yan, L. (2013). Proteomic mechanisms of cardioprotection during Mammalian hibernation in woodchucks, Marmota Monax. Journal of Proteome Research, 12, 4221-4229. http://doi.org/10.1021/pr400580f

Lin, Z., Murtaza, I., Wang, K., Jiao, J., Gao, J., \& Li, P. F. (2009). miR-23a functions downstream of NFATc3 to regulate cardiac hypertrophy. Proceedings of the National Academy of Sciences of the United States of America, 106(29), 12103-12108. http://doi.org/10.1073/pnas.0811371106

Liu, B., Wang, L. C., \& Belke, D. D. (1991). Effect of low temperature on the cytosolic free $\mathrm{Ca} 2+$ in rat ventricular myocytes. Cell Calcium, 12(1), 11-18. 
Liu, B., Wang, L. C. H., \& Belke, D. D. (1993). Effects of Temperature and pH on Cardiac Myofilament Ca-2+ Sensitivity in Rat and Ground Squirrel. Am J Physiol, 264(1 Part 2), R104-R108.

Liu, B., Wohlfart, B., \& Johansson, B. W. (1990). Effects of low temperature on contraction in papillary muscles from rabbit, rat, and hedgehog. Cryobiology, 27(5), 539-546. http://doi.org/10.1016/0011-2240(90)90041-2

Liu, Q., Wilkins, B. J., Lee, Y. J., Ichijo, H., \& Molkentin, J. D. (2006). Direct interaction and reciprocal regulation between ASK1 and calcineurin-NFAT control cardiomyocyte death and growth. Molecular and Cellular Biology, 26(10), 3785-3797. http://doi.org/10.1128/MCB.26.10.3785

Lorson, C. L., Rindt, H., \& Shababi, M. (2010). Spinal muscular atrophy: Mechanisms and therapeutic strategies. Human Molecular Genetics, 19(1), 111-118. http://doi.org/10.1093/hmg/ddq147

MacDonald, J. A., \& Storey, K. B. (2005). Temperature and phosphate effects on allosteric phenomena of phosphofructokinase from a hibernating ground squirrel (Spermophilus lateralis). FEBS Journal, 272(1), 120-128. http://doi.org/10.1111/j.14321033.2004.04388.x

MacPherson, P. C. D., Wang, X., \& Goldman, D. (2011). Myogenin regulates denervationdependent muscle atrophy in mouse soleus muscle. Journal of Cellular Biochemistry, 112(8), 2149-2159. http://doi.org/10.1002/jcb.23136

Malatesta, M., Perdoni, F., Battistelli, S., Muller, S., \& Zancanaro, C. (2009). The cell nuclei of skeletal muscle cells are transcriptionally active in hibernating edible dormice. BMC Cell 
Biology, 10, 19. http://doi.org/10.1186/1471-2121-10-19

Mamady, H., \& Storey, K. B. (2006). Up-regulation of the endoplasmic reticulum molecular chaperone GRP78 during hibernation in thirteen-lined ground squirrels. Molecular and Cellular Biochemistry, 292, 89-98. http://doi.org/10.1007/s11010-006-9221-8

Marston, S. B., \& Hodgkinson, J. L. (2001). Cardiac and skeletal myopathies: Can genotype explain phenotype? Journal of Muscle Research and Cell Motility, 22(1), 1-4. http://doi.org/10.1023/A:1010355716511

Matsuzaki, H., Ichino, A., Hayashi, T., Yamamoto, T., \& Kikkawa, U. (2005). Regulation of intracellular localization and transcriptional activity of FOXO4 by protein kinase $\mathrm{B}$ through phosphorylation at the motif sites conserved among the FOXO family. Journal of Biochemistry, 138(4), 485-491. http://doi.org/10.1093/jb/mvi146

McArthur, M. D., \& Milsom, W. K. (1991). Changes in ventilation and respiratory sensitivity associated with hibernation in Columbian (Spermophilus columbianus) and golden-mantled (Spermophilus lateralis) ground squirrels. Physiological Zoology, 64(4), 940-959.

McMullen, D. C., \& Hallenbeck, J. M. (2010). Regulation of Akt during torpor in the hibernating ground squirrel, Ictidomys tridecemlineatus. Journal of Comparative Physiology B:

Biochemical, Systemic, and Environmental Physiology, 180, 927-934. http://doi.org/10.1007/s00360-010-0468-8

Millay, D. P., O’Rourke, J. R., Sutherland, L. B., Bezprozvannaya, S., Shelton, J. M., BasselDuby, R., \& Olson, E. N. (2013). Myomaker is a membrane activator of myoblast fusion and muscle formation. (Supp). Nature, 499, 301-305. http://doi.org/10.1038/nature12343 
Millay, D. P., Sutherland, L. B., Bassel-duby, R., \& Olson, E. N. (2014). Myomaker is essential for muscle regeneration Myomaker is essential for muscle regeneration, 1641-1646. http://doi.org/10.1101/gad.247205.114

Molkentin, J. D., Lu, J. R., Antos, C. L., Markham, B., Richardson, J., Robbins, J., ... Olson, E. N. (1998). A calcineurin-dependent transcriptional pathway for cardiac hypertrophy. Cell, 93, 215-228. http://doi.org/10.1016/S0092-8674(00)81573-1

Moresi, V., Williams, A. H., Meadows, E., Flynn, J. M., Potthoff, M. J., McAnally, J., ... Olson, E. N. (2010). Myogenin and class II HDACs control neurogenic muscle atrophy by inducing E3 ubiquitin ligases. Cell, 143(1), 35-45. http://doi.org/10.1016/j.cell.2010.09.004

Morin, P., Ni, Z., McMullen, D. C., \& Storey, K. B. (2008). Expression of Nrf2 and its downstream gene targets in hibernating 13-lined ground squirrels, Spermophilus tridecemlineatus. Molecular and Cellular Biochemistry, 312, 121-129. http://doi.org/10.1007/s11010-008-9727-3

Morin, P., \& Storey, K. B. (2006). Evidence for a reduced transcriptional state during hibernation in ground squirrels. Cryobiology, 53, 310-318. http://doi.org/10.1016/j.cryobiol.2006.08.002

Moylan, J. S., Smith, J. D., Chambers, M. a, McLoughlin, T. J., \& Reid, M. B. (2008). TNF induction of atrogin-1/MAFbx mRNA depends on Foxo4 expression but not AKT-Foxo1/3 signaling. American Journal of Physiology. Cell Physiology, 295, C986-C993. http://doi.org/10.1152/ajpcell.00041.2008

Nakipova, O. V., Zakharova, N. M., Andreeva, L. A., Chumaeva, N. A., Averin, A., Kosarskii, L. S., ... Pieske, B. (2007). The seasonal peculiarities of force-frequency relationships in 
active ground squirrel Spermophilus undulatus ventricle. Cryobiology, 55, 173-181. http://doi.org/10.1016/j.cryobiol.2007.07.001

Neel, N. F., Martin, T. D., Stratford, J. K., Zand, T. P., Reiner, D. J., \& Der, C. J. (2011). The RalGEF-Ral Effector Signaling Network: The Road Less Traveled for Anti-Ras Drug Discovery. Genes \& Cancer, 2(3), 275-287. http://doi.org/10.1177/1947601911407329

Nelson, O. L., \& Rourke, B. C. (2013). Increase in cardiac myosin heavy-chain (MyHC) alpha protein isoform in hibernating ground squirrels, with echocardiographic visualization of ventricular wall hypertrophy and prolonged contraction. The Journal of Experimental Biology, 216, 4678-4690. http://doi.org/10.1242/jeb.088773

Novák, A., Boldizsár, Á., Ádám É., Kozma-Bognár, L., Majláth I., Båga M., ... Galba G. (2015). Light-quality and temperature-dependentCBF14 gene expression modulates freezing tolerance in cereals. Journal of Experimental Botany, (December), 1102-1105.

Osborne, P. G., \& Hashimoto, M. (2006). Brain antioxidant levels in hamsters during hibernation, arousal and cenothermia. Behavioural Brain Research, 168, 208-214. http://doi.org/10.1016/j.bbr.2005.11.007

Paula-Gomes, S., Gonçalves, D. A. P., Baviera, A. M., Zanon, N. M., Navegantes, L. C. C., \& Kettelhut, I. C. (2013). Insulin suppresses atrophy- And autophagy-Related genes in heart tissue and cardiomyocytes through AKT/FOXO signaling. Hormone and Metabolic Research, 45, 849-855. http://doi.org/10.1055/s-0033-1347209

Park, S., Uesugi, M., \& Verdine, G. L. (2000). A second calcineurin binding site on the NFAT regulatory domain. Proceedings of the National Academy of Sciences of the United States of America, 97(13), 7130-7135. http://doi.org/10.1073/pnas.97.13.7130 
Pusl, T., Wu, J. J., Zimmerman, T. L., Zhang, L., Ehrlich, B. E., Berchtold, M. W., ... Bennett, A. M. (2002). Epidermal growth factor-mediated activation of the ETS domain transcription factor Elk-1 requires nuclear calcium. Journal of Biological Chemistry, 277(30), 27517-27527. http://doi.org/10.1074/jbc.M203002200

Qin, W., Pan, J., Wu, Y., Bauman, W. A., \& Cardozo, C. (2015). Anabolic steroids activate calcineurin-NFAT signaling and thereby increase myotube size and reduce denervation atrophy. Molecular and Cellular Endocrinology, 399, 336-345. http://doi.org/10.1016/j.mce.2014.09.025

Rao, A., Luo, C., \& Hogan, P. G. (1997). Transcription factors of the NFAT family: regulation and function. Annual Review of Immunology, 15, 707-747. http://doi.org/10.1146/annurev.immunol.15.1.707

Riehle, M. M., Bennett, A. F., Lenski, R. E., \& Long, A. D. (2003). Evolutionary changes in heat-inducible gene expression in lines of Escherichia coli adapted to high temperature. Physiological Genomics, 14(1), 47-58. http://doi.org/10.1152/physiolgenomics.00034.2002

Rourke, B. C., Yokoyama, Y., Milsom, W. K., \& Caiozzo, V. J.. (2004). Myosin Isoform Expression and MAFbx mRNA Levels in Hibernating Golden-Mantled Ground Squirrels (Spermophilus lateralis). Physiological and Biochemical Zoology: Ecological and Evolutionary Approaches, 77(4), 582-593.

Rusnak, F., \& Mertz, P. (2000). Calcineurin: form and function. Physiological Reviews, 80, 1483-1521. http://doi.org/10.1172/JCI57909.date

Sandri, M., Sandri, C., Gilbert, A., Skurk, C., Calabria, E., Picard, A., ... Goldberg, A. L. (2004). Foxo transcription factors induce the atrophy-related ubiquitin ligase atrogin-1 and cause 
skeletal muscle atrophy. Cell, 117(3), 399-412. http://doi.org/10.1016/S00928674(04)00400-3

Schiaffino, S., Dyar, K. A., Ciciliot, S., Blaauw, B., \& Sandri, M. (2013). Mechanisms regulating skeletal muscle growth and atrophy. The FEBS Journal, 280, 4294-4314. http://doi.org/10.1111/febs. 12253

Schiaffino, S., Sandri, M., \& Murgia, M. (2007). Activity-dependent signaling pathways controlling muscle diversity and plasticity. Physiology (Bethesda, Md.), 22, 269-278. http://doi.org/10.1152/physiol.00009.2007

Schubert, W., Yang, X. Y., Yang, T. T. C., Factor, S. M., Lisanti, M. P., Molkentin, J. D., ... Chow, C.W. (2003). Requirement of transcription factor NFAT in developing atrial myocardium. The Journal of Cell Biology, 161(5), 861-874. http://doi.org/10.1083/jcb.200301058

Shibasaki, F., Hallin, U., \& Uchino, H. (2002). Calcineurin as a multifunctional regulator. Journal of Biochemistry, 131(1), 1-15.

Shioda, N., Moriguchi, S., Shirasaki, Y., \& Fukunaga, K. (2006). Generation of constitutively active calcineurin by calpain contributes to delayed neuronal death following mouse brain ischemia. Journal of Neurochemistry, 98(1), 310-320. http://doi.org/10.1111/j.14714159.2006.03874.x

Stenvinkel, P., Fröbert, O., Anderstam, B., Palm, F., Eriksson, M., Bragfors-Helin, A. C., ... Johnson, R. J. (2013). Metabolic Changes in Summer Active and Anuric Hibernating FreeRanging Brown Bears (Ursus arctos). PLoS ONE, 8(9). http://doi.org/10.1371/journal.pone.0072934 
Stitt, T. N., Drujan, D., Clarke, B. A., Panaro, F., Timofeyva, Y., Kline, W. O., ... Glass, D. J. (2004). The IGF-1/PI3K/Akt pathway prevents expression of muscle atrophy-induced ubiquitin ligases by inhibiting FOXO transcription factors. Molecular Cell, 14(3), 395-403. http://doi.org/10.1016/S1097-2765(04)00211-4

Storey, K. B. (2010). Out cold: Biochemical regulation of mammalian hibernation - A minireview. Gerontology. 56, 220-230. doi: 10.1159/000228829

Storey, K. B., \& Storey, J. M. (2004). Metabolic rate depression in animals: transcriptional and translational controls. Biological Reviews of the Cambridge Philosophical Society, 79, 207233. http://doi.org/10.1017/S1464793103006195

Storey, K. B., \& Storey, J. M. (2010). Metabolic rate depression. The biochemistry of mammalian hibernation. Advances in Clinical Chemistry, 52(C), 77-108. http://doi.org/10.1016/S0065-2423(10)52003-1

Swindell, W. R., Huebner, M., \& Weber, A. P. (2007). Plastic and adaptive gene expression patterns associated with temperature stress in Arabidopsis thaliana. Heredity, 99(2), 143150. http://doi.org/10.1038/sj.hdy.6800975

Takaishi, H., Konishi, H., Matsuzaki, H., Ono, Y., Shirai, Y., Saito, N., ... Nishizuka, Y. (1999). Regulation of nuclear translocation of forkhead transcription factor AFX by protein kinase B. Proceedings of the National Academy of Sciences of the United States of America, 96(21), 11836-11841.

Tang, E. D., Nuñez, G., Barr, F. G., \& Guan, K. L. (1999). Negative regulation of the forkhead transcription factor FKHR by Akt. Journal of Biological Chemistry, 274(24), 16741-16746. http://doi.org/10.1074/jbc.274.24.16741 
Tessier, S. N., \& Storey, K. B. (2010). Expression of myocyte enhancer factor-2 and downstream genes in ground squirrel skeletal muscle during hibernation. Molecular and Cellular Biochemistry, 344, 151-162. http://doi.org/10.1007/s11010-010-0538-y

Tessier, S. N., \& Storey, K. B. (2012). Myocyte enhancer factor-2 and cardiac muscle gene expression during hibernation in thirteen-lined ground squirrels. Gene, 501(1), 8-16. http://doi.org/10.1016/j.gene.2012.04.004

Tessier, S. N., \& Storey, K. B. (2016). Lessons from mammalian hibernators: molecular insights into striated muscle plasticity and remodeling. Biomolecular Concepts, $0(0), 1-24$. http://doi.org/10.1515/bmc-2015-0031

Thompson, M., Andrade, V. A., Andrade, S. J., Pusl, T., Ortega, J. M., Goes, A. M., \& Leite, M. F. (2003). Inhibition of the TEF/TEAD transcription factor activity by nuclear calcium and distinct kinase pathways. Biochemical and Biophysical Research Communications, 301(2), 267-274. http://doi.org/10.1016/S0006-291X(02)03024-3

Turtoi, A., Blomme, A., Bellahcène, A., Gilles, C., Hennequierère, V., Peixoto, P., ... Castronovo, V. (2013). Myoferlin is a key regulator of EGFR activity in breast cancer. Cancer Research, 73(17), 5438-5448. http://doi.org/10.1158/0008-5472.CAN-13-1142

Van Den Berg, M. C. W., Van Gogh, I. J. A., Smits, A. M. M., Van Triest, M., Dansen, T. B., Visscher, M., ... Burgering, B. M. T. (2013). The small GTPase RALA controls c-Jun Nterminal kinase-mediated FOXO activation by regulation of a JIP1 scaffold complex. Journal of Biological Chemistry, 288(30), 21729-21741. http://doi.org/10.1074/jbc.M113.463885

van der Horst, A., \& Burgering, B. M. T. (2007). Stressing the role of FoxO proteins in lifespan 
and disease. Nature Reviews. Molecular Cell Biology, 8(6), 440-450.

http://doi.org/10.1038/nrm2190

Waddell, D. S., Baehr, L. M., van den Brandt, J., Johnsen, S. A., Reichardt, H. M., Furlow, J. D., \& Bodine, S. C. (2008). The glucocorticoid receptor and FOXO1 synergistically activate the skeletal muscle atrophy-associated MuRF1 gene. American Journal of Physiology. Endocrinology and Metabolism, 295(4), E785-E797. http://doi.org/10.1152/ajpendo.00646.2007

Wang, L. C. H., \& Lee, T. F. (2011). Torpor and Hibernation in Mammals: Metabolic , Physiological, and Biochemical Adaptations. In Compr Physiol (pp. 507-532). http://doi.org/10.1002/cphy.cp040122

Wang, S. Q., Lakatta, E. G., Cheng, H., \& Zhou, Z. Q. (2002). Adaptive mechanisms of intracellular calcium homeostasis in mammalian hibernators. The Journal of Experimental Biology, 205(Pt 19), 2957-2962.

Wang, S. Q., \& Zhou, Z. Q. (1999). Alpha-stat calibration of indo-1 fluorescence and measurement of intracellular free calcium in rat ventricular cells at different temperatures. Life Sci, 65(9), 871-877.

Wang, S., Zhou, Z., \& Qian, H. (1999). Temperature dependence of intracellular free calcium in cardiac myocytes from rat and ground squirrel measured by confocal microscopy. Science in China. Series C, Life Sciences / Chinese Academy of Sciences, 42(3), 293-299. http://doi.org/10.1007/BF03183606

Wang, S., Zhou, Z., \& Qian, H. (2000). Recording of calcium transient and analysis of calcium removal mechanisms in cardiac myocytes from rats and ground squirrels. Science in China. 
Series C, Life Sciences / Chinese Academy of Sciences, 43(2), 191-199.

http://doi.org/10.1007/BF02879128

Wang, S. Q., Huang, Y. H., Liu, K. S., \& Zhou, Z. Q. (1997). Dependence of myocardial hypothermia tolerance on sources of activator calcium. Cryobiology, 35, 193-200. http://doi.org/10.1006/cryo.1997.2040

Wickler, S. J., Hoyt, D. F., \& van Breukelen, F. (1991). Disuse atrophy in the hibernating golden-mantled ground squirrel, Spermophilus lateralis. The American Journal of Physiology, 261, R1214-R1217.

Wu, C. W., \& Storey, K. B. (2012). Pattern of cellular quiescence over the hibernation cycle in liver of thirteen-lined ground squirrels. Cell Cycle, 11(9), 1714-1726. http://doi.org/10.4161/cc.19799

Wu, C. W., \& Storey, K. B. (2014). FoxO3a-mediated activation of stress responsive genes during early torpor in a mammalian hibernator. Molecular and Cellular Biochemistry, 390, 185-195. http://doi.org/10.1007/s11010-014-1969-7

Wu, H. Y., Tomizawa, K., Oda, Y., Wei, F. Y., Lu, Y. F., Matsushita, M., ... Matsui, H. (2004). Critical Role of Calpain-mediated Cleavage of Calcineurin in Excitotoxic Neurodegeneration. Journal of Biological Chemistry, 279(6), 4929-4940. http://doi.org/10.1074/jbc.M309767200

Xiong, X., Tao, R., DePinho, R. A., \& Dong, X. C. (2012). The autophagy-related gene 14 (Atg14) is regulated by forkhead box $\mathrm{O}$ transcription factors and circadian rhythms and plays a critical role in hepatic autophagy and lipid metabolism. Journal of Biological Chemistry, 287, 39107-39114. http://doi.org/10.1074/jbc.M112.412569 
Xu, R., Andres-Mateos, E., Mejias, R., MacDonald, E. M., Leinwand, L. A., Merriman, D. K., ... Cohn, R. D. (2013). Hibernating squirrel muscle activates the endurance exercise pathway despite prolonged immobilization. Experimental Neurology, 247, 392-401. http://doi.org/10.1016/j.expneurol.2013.01.005

Yan, L., Kudej, R. K., Vatner, D. E., \& Vatner, S. F. (2015). Myocardial ischemic protection in natural mammalian hibernation. Basic Research in Cardiology, 110, 1-10. http://doi.org/10.1007/s00395-015-0462-0

Yancey, P. H., \& Burg, M. B. (1990). Counteracting effects of urea and betaine in mammalian cells in culture. American Journal of Physiology, 258(1 Pt 2), R198-204.

Yang, S., \& Klee, C. B. (2000). Low affinity Ca2+-binding sites of calcineurin B mediate conformational changes in calcineurin A. Biochemistry, 39(51), 16147-16154.

Yang, T., Davis, R. J., \& Chow, C. W. (2001). Requirement of Two NFATc4 Transactivation Domains for CBP Potentiation. Journal of Biological Chemistry, 276(43), 39569-39576. http://doi.org/10.1074/jbc.M102961200

Yeh, J. J., \& Der, C. J. (2007). Targeting signal transduction in pancreatic cancer treatment. Expert Opinion on Therapeutic Targets, 11(5), 673-694. http://doi.org/10.1517/14728222.11.5.673

Yu, C., Sharma, A., Trane, A., Utokaparch, S., Leung, C., \& Bernatchez, P. (2011). Myoferlin gene silencing decreases Tie-2 expression in vitro and angiogenesis in vivo. Vascular Pharmacology, 55(1-3), 26-33. http://doi.org/10.1016/j.vph.2011.04.001

Yuan, Z., Becker, E. B. E., Merlo, P., Yamada, T., DiBacco, S., Konishi, Y., ... Bonni, A. 
(2008). Activation of FOXO1 by Cdk1 in cycling cells and postmitotic neurons. Science (New York, N.Y.), 319(5870), 1665-1668. http://doi.org/10.1126/science.1152337

Zhang, X. H., Yokoo, H., Nishioka, H., Fujii, H., Matsuda, N., Hayashi, T., \& Hattori, Y. (2010). Beneficial effect of the oligomerized polyphenol oligonol on high glucose-induced changes in eNOS phosphorylation and dephosphorylation in endothelial cells. British Journal of Pharmacology, 159(4), 928-938. http://doi.org/10.1111/j.1476-5381.2009.00594.x

Zhang, Y., \& Storey, K. B. (2015). Expression of nuclear factor of activated T cells (NFAT) and downstream muscle-specific proteins in ground squirrel skeletal and heart muscle during hibernation. Molecular and Cellular Biochemistry, 412(1), 27-40.

http://doi.org/10.1007/s11010-015-2605-x 
Appendices 


\section{Appendix A: Antibodies Used for Western Blotting}

Protein Target
NFATc1
NFATc2
NFATc3
NFATc4
Myomaker/TMEM8c
Myoferlin
Foxo1
Foxo3a
p-Foxo1 S319
p-Foxo1 T24/p-Foxo3a T32
p-Foxo3a S318/321
Foxo4
p-Foxo4 S197
p-Foxo4 T451
MAFbx
MURF1
MyoG
Calcineurin A
CAM
Calpain-1
Ralbp1
Ral A
Ras

\begin{tabular}{|c|c|}
\hline$\underline{\text { Supplier }}$ & $\underline{\text { Associated Product Code }}$ \\
\hline Santa Cruz & sc-13033 \\
\hline Santa Cruz & sc-13024 \\
\hline Santa Cruz & sc- 8321 \\
\hline Santa Cruz & sc-13036 \\
\hline Santa Cruz & sc-244460 \\
\hline Santa Cruz & sc-134798 \\
\hline Genetex & GTX110724 \\
\hline Genetex & GTX100277 \\
\hline Genescript & A00373 \\
\hline Cell Signaling & 9464P \\
\hline Cell Signaling & 9465 \\
\hline Cell Signaling & 9472 \\
\hline Santa Cruz & sc-101628 \\
\hline Signalway Antibody & 12053 \\
\hline Santa Cruz & sc-27645 \\
\hline Genetex & GTX110475 \\
\hline Santa Cruz & sc-576 \\
\hline Genetex & GTX111039 \\
\hline Upstate Biotechnology & 06-396 \\
\hline Genetex & GTX102340 \\
\hline Genetex & GTX132480 \\
\hline Genetex & GTX114204 \\
\hline Signalway Antibody & 38202 \\
\hline
\end{tabular}


$\underline{\text { Appendix B: Western Blotting Conditions }}$

\begin{tabular}{|c|c|c|c|c|c|c|c|}
\hline $\begin{array}{c}1^{\circ} \\
\text { antibody } \\
\text { target }\end{array}$ & Tissue & $\begin{array}{c}\text { Protein } \\
\text { molecular } \\
\text { weight } \\
(\mathbf{k D a})\end{array}$ & $\begin{array}{c}\text { Gel } \\
\text { percent } \\
(\%)\end{array}$ & $\begin{array}{c}\text { Transfer } \\
\text { conditions } \\
\text { and time (h) }\end{array}$ & $\begin{array}{l}\text { Block } \\
\text { with } \\
\text { milk } \\
(\%)\end{array}$ & $\begin{array}{l}1^{\circ} \text { antibody } \\
\text { incubation }\end{array}$ & $\begin{array}{l}2^{\circ} \text { antibody } \\
\text { incubation }\end{array}$ \\
\hline \multirow[t]{2}{*}{ NFATc1 } & Muscle & 101 & 6 & $160 \mathrm{~mA}, 3 \mathrm{~h}$ & 2.5 & 1:500 overnight & Rabbit 1:6000 $30 \mathrm{~min}$ \\
\hline & Heart & 101 & 6 & $160 \mathrm{~mA}, 3 \mathrm{~h}$ & 2.5 & 1:500 overnight & Rabbit 1:6000 $30 \mathrm{~min}$ \\
\hline \multirow[t]{2}{*}{ NFATc2 } & Muscle & 92 & 6 & $160 \mathrm{~mA}, 3 \mathrm{~h}$ & 2.5 & 1:500 overnight & Rabbit 1:6000 $30 \mathrm{~min}$ \\
\hline & Heart & 92 & 6 & $160 \mathrm{~mA}, 3 \mathrm{~h}$ & 2.5 & 1:500 overnight & Rabbit 1:6000 $30 \mathrm{~min}$ \\
\hline \multirow[t]{2}{*}{ NFATc3 } & Muscle & 130 & 6 & $160 \mathrm{~mA}, 3 \mathrm{~h}$ & 2.5 & 1:500 overnight & Rabbit 1:6000 $30 \mathrm{~min}$ \\
\hline & Heart & 130 & 6 & $160 \mathrm{~mA}, 3 \mathrm{~h}$ & 2.5 & 1:500 overnight & Rabbit 1:6000 $30 \mathrm{~min}$ \\
\hline \multirow[t]{2}{*}{ NFATc4 } & Muscle & 85 & 6 & $160 \mathrm{~mA}, 3 \mathrm{~h}$ & 2.5 & 1:500 overnight & Rabbit 1:6000 $30 \mathrm{~min}$ \\
\hline & Heart & 85 & 6 & $160 \mathrm{~mA}, 3 \mathrm{~h}$ & 2.5 & 1:500 overnight & Rabbit 1:6000 $30 \mathrm{~min}$ \\
\hline \multirow{2}{*}{$\begin{array}{l}\text { Myomaker/ } \\
\text { TMEM8c }\end{array}$} & Muscle & 235 & 15 & $160 \mathrm{~mA}, 1.5 \mathrm{~h}$ & 5 & 1:500 overnight & Goat 1:6000 $30 \mathrm{~min}$ \\
\hline & Heart & 235 & 15 & $160 \mathrm{~mA}, 1.5 \mathrm{~h}$ & 5 & 1:500 overnight & Goat 1:6000 $30 \mathrm{~min}$ \\
\hline \multirow[t]{2}{*}{ Myoferlin } & Muscle & 25 & 6 & $160 \mathrm{~mA}, 3 \mathrm{~h}$ & 5 & 1:500 overnight & Rabbit 1:6000 $30 \mathrm{~min}$ \\
\hline & Heart & 25 & 6 & $160 \mathrm{~mA}, 3 \mathrm{~h}$ & 5 & 1:500 overnight & Rabbit 1:6000 $30 \mathrm{~min}$ \\
\hline \multirow[t]{2}{*}{ Foxo1 } & Muscle & 68 & 8 & $160 \mathrm{~mA}, 1.5 \mathrm{~h}$ & 5 & $1: 1000$ overnight & Rabbit 1:6000 $30 \mathrm{~min}$ \\
\hline & Heart & 68 & 8 & $160 \mathrm{~mA}, 1.5 \mathrm{~h}$ & 5 & $1: 1000$ overnight & Rabbit 1:6000 $30 \mathrm{~min}$ \\
\hline \multirow[t]{2}{*}{ Foxo3a } & Muscle & 90 & 8 & $160 \mathrm{~mA}, 1.5 \mathrm{~h}$ & 5 & $1: 1000$ overnight & Rabbit 1:6000 $30 \mathrm{~min}$ \\
\hline & Heart & 90 & 8 & $160 \mathrm{~mA}, 1.5 \mathrm{~h}$ & 5 & $1: 1000$ overnight & Rabbit 1:6000 $30 \mathrm{~min}$ \\
\hline \multirow{2}{*}{$\begin{array}{c}\text { p-Foxo1 } \\
\text { S319 }\end{array}$} & Muscle & 68 & 8 & $160 \mathrm{~mA}, 1.5 \mathrm{~h}$ & 5 & $1: 1000$ overnight & Rabbit 1:6000 $30 \mathrm{~min}$ \\
\hline & Heart & 68 & 8 & $160 \mathrm{~mA}, 1.5 \mathrm{~h}$ & 5 & $1: 1000$ overnight & Rabbit 1:6000 $30 \mathrm{~min}$ \\
\hline \multirow{3}{*}{$\begin{array}{c}\mathrm{p}-\text { Foxo1 } \\
\text { T24/p- } \\
\text { Foxo3a } \\
\text { T32 }\end{array}$} & Muscle & $\begin{array}{c}\text { Foxo1: } 69 \\
\text { Foxo3a: } \\
95\end{array}$ & 8 & $160 \mathrm{~mA}, 1.5 \mathrm{~h}$ & 5 & $1: 1000$ overnight & Rabbit 1:6000 $30 \mathrm{~min}$ \\
\hline & Heart & $69 / 95$ & 8 & $160 \mathrm{~mA}, 1.5 \mathrm{~h}$ & 5 & $1: 1000$ overnight & Rabbit 1:6000 $30 \mathrm{~min}$ \\
\hline & Muscle & 72 & 8 & $160 \mathrm{~mA}, 1.5 \mathrm{~h}$ & 5 & $1: 1000$ overnight & Rabbit 1:6000 $30 \mathrm{~min}$ \\
\hline
\end{tabular}




\begin{tabular}{|c|c|c|c|c|c|c|c|}
\hline $\begin{array}{l}\text { p-Foxo3a } \\
\text { S318/321 }\end{array}$ & Heart & 72 & 8 & $160 \mathrm{~mA}, 1.5 \mathrm{~h}$ & 5 & 1:1000 overnight & Rabbit 1:6000 $30 \mathrm{~min}$ \\
\hline \multirow[t]{2}{*}{ Foxo4 } & Muscle & 53 & 8 & $160 \mathrm{~mA}, 1.5 \mathrm{~h}$ & 7.5 & 1:1000 overnight & Rabbit 1:6000 $30 \mathrm{~min}$ \\
\hline & Heart & 53 & 8 & $160 \mathrm{~mA}, 1.5 \mathrm{~h}$ & 7.5 & 1:1000 overnight & Rabbit 1:6000 $30 \mathrm{~min}$ \\
\hline \multirow{2}{*}{$\begin{array}{c}\text { p-Foxo4 } \\
\text { S197 }\end{array}$} & Muscle & 53 & 10 & $160 \mathrm{~mA}, 1.5 \mathrm{~h}$ & 5 & 1:1000 overnight & Rabbit 1:6000 $30 \mathrm{~min}$ \\
\hline & Heart & 53 & 10 & $160 \mathrm{~mA}, 1.5 \mathrm{~h}$ & 5 & 1:1000 overnight & Rabbit 1:6000 $30 \mathrm{~min}$ \\
\hline \multirow{2}{*}{$\begin{array}{c}\text { p-Foxo4 } \\
\text { T451 }\end{array}$} & Muscle & 53 & 8 & $160 \mathrm{~mA}, 1.5 \mathrm{~h}$ & 5 & 1:1000 overnight & Rabbit 1:6000 $30 \mathrm{~min}$ \\
\hline & Heart & 53 & 8 & $160 \mathrm{~mA}, 1.5 \mathrm{~h}$ & 5 & 1:1000 overnight & Rabbit 1:6000 $30 \mathrm{~min}$ \\
\hline \multirow[t]{2}{*}{ MAFbx } & Muscle & 42 & 10 & $160 \mathrm{~mA}, 1.5 \mathrm{~h}$ & 7.5 & 1:1000 overnight & Rabbit 1:6000 $30 \mathrm{~min}$ \\
\hline & Heart & 42 & 10 & $160 \mathrm{~mA}, 1.5 \mathrm{~h}$ & 7.5 & 1:1000 overnight & Rabbit 1:6000 $30 \mathrm{~min}$ \\
\hline \multirow[t]{2}{*}{ MURF1 } & Muscle & 40 & 8 & $160 \mathrm{~mA}, 1.5 \mathrm{~h}$ & 7.5 & 1:1000 overnight & Rabbit 1:6000 $30 \mathrm{~min}$ \\
\hline & Heart & 40 & 8 & $160 \mathrm{~mA}, 1.5 \mathrm{~h}$ & 7.5 & 1:1000 overnight & Rabbit 1:6000 $30 \mathrm{~min}$ \\
\hline \multirow[t]{2}{*}{ MyoG } & Muscle & 34 & 10 & $160 \mathrm{~mA}, 1.5 \mathrm{~h}$ & 7.5 & 1:1000 overnight & Rabbit 1:6000 $30 \mathrm{~min}$ \\
\hline & Heart & 34 & 10 & $160 \mathrm{~mA}, 1.5 \mathrm{~h}$ & 7.5 & 1:1000 overnight & Rabbit 1:6000 $30 \mathrm{~min}$ \\
\hline \multirow{2}{*}{$\begin{array}{c}\text { Calcineurin } \\
\text { A }\end{array}$} & Muscle & 59 & 8 & $160 \mathrm{~mA}, 1.5 \mathrm{~h}$ & 5 & 1:1000 overnight & Rabbit 1:6000 $30 \mathrm{~min}$ \\
\hline & Heart & 59 & 8 & $160 \mathrm{~mA}, 1.5 \mathrm{~h}$ & 5 & 1:1000 overnight & Rabbit 1:6000 $30 \mathrm{~min}$ \\
\hline \multirow[t]{2}{*}{ CAM } & Muscle & 19 & 15 & $30 \mathrm{~V} 1.5 \mathrm{~h}$ & 5 & 1:1000 overnight & Rabbit 1:6000 $30 \mathrm{~min}$ \\
\hline & Heart & 19 & 15 & $30 \mathrm{~V} 1.5 \mathrm{~h}$ & 5 & 1:1000 overnight & Rabbit 1:6000 $30 \mathrm{~min}$ \\
\hline \multirow[t]{2}{*}{ Calpain-1 } & Muscle & 82 & 8 & $160 \mathrm{~mA}, 1.5 \mathrm{~h}$ & 5 & 1:1000 overnight & Rabbit 1:6000 $30 \mathrm{~min}$ \\
\hline & Heart & 82 & 8 & $160 \mathrm{~mA}, 1.5 \mathrm{~h}$ & 5 & 1:1000 overnight & Rabbit 1:6000 $30 \mathrm{~min}$ \\
\hline \multirow[t]{2}{*}{ Ras } & Muscle & 21 & 15 & $30 \mathrm{~V} 1.5 \mathrm{~h}$ & 7.5 & 1:1000 overnight & Rabbit 1:6000 $30 \mathrm{~min}$ \\
\hline & Heart & 21 & 15 & $30 \mathrm{~V} 1.5 \mathrm{~h}$ & 7.5 & 1:1000 overnight & Rabbit 1:6000 $30 \mathrm{~min}$ \\
\hline \multirow[t]{2}{*}{ Ral A } & Muscle & 24 & 15 & $30 \mathrm{~V} 1.5 \mathrm{~h}$ & 7.5 & 1:1000 overnight & Rabbit 1:6000 $30 \mathrm{~min}$ \\
\hline & Heart & 24 & 15 & $30 \mathrm{~V} 1.5 \mathrm{~h}$ & 7.5 & 1:1000 overnight & Rabbit 1:6000 $30 \mathrm{~min}$ \\
\hline \multirow[t]{2}{*}{ Ralbp1 } & Muscle & 74 & 10 & $160 \mathrm{~mA}, 1.5 \mathrm{~h}$ & 5 & 1:1000 overnight & Rabbit 1:6000 $30 \mathrm{~min}$ \\
\hline & Heart & 74 & 10 & $160 \mathrm{~mA}, 1.5 \mathrm{~h}$ & 5 & 1:1000 overnight & Rabbit 1:6000 $30 \mathrm{~min}$ \\
\hline
\end{tabular}




\section{Appendix C: Summary Figures and Tables}

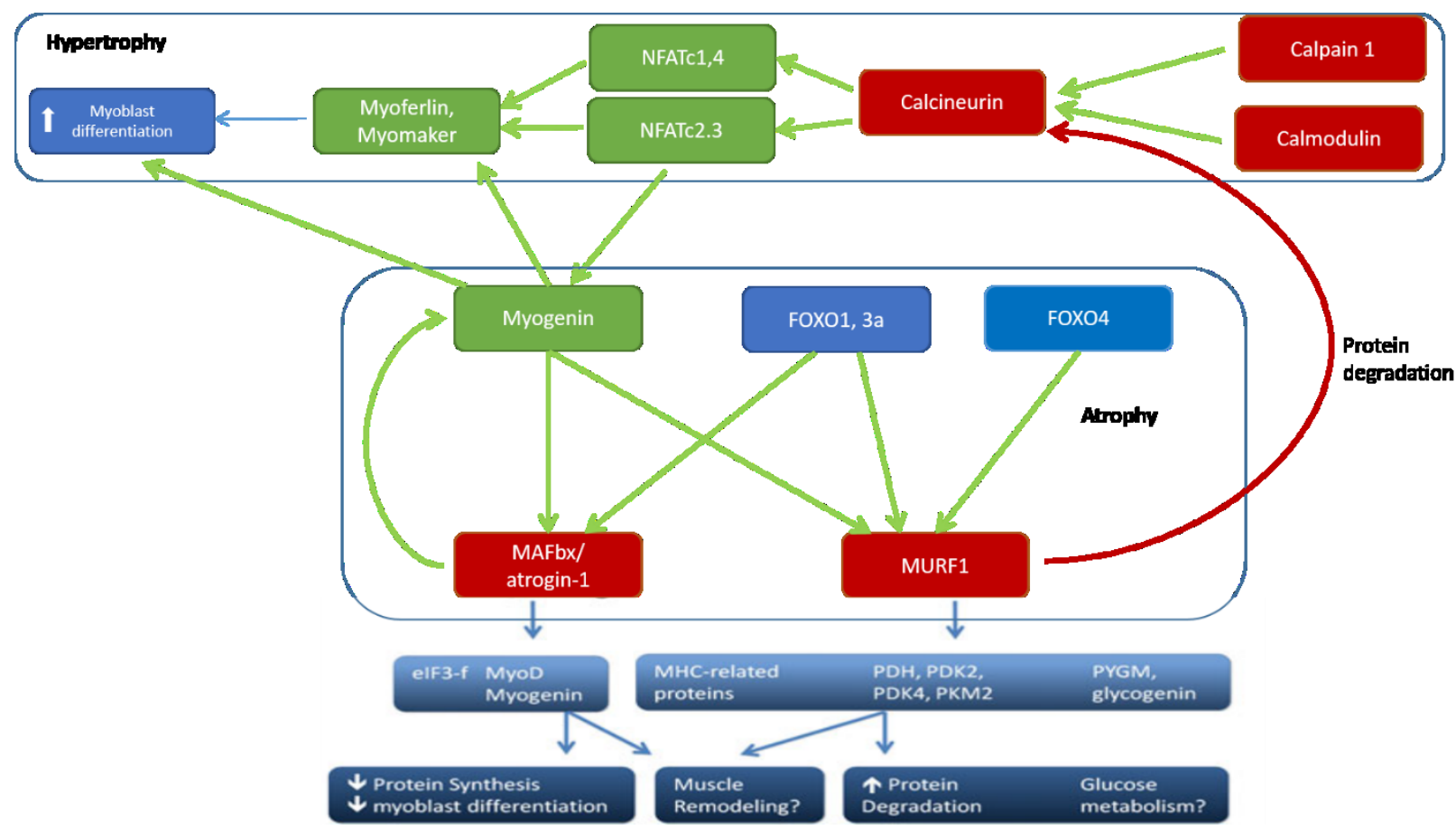

Supplementary Figure 1: Summary figure of the relationship between the targets analyzed in the current thesis. The NFAT-calcineurin pathway shown at the top of the figure regulates the downstream muscle proteins Myoferlin and Myomaker, which may result in increased myoblast differentiation. The transcription factors Myogenin and the Foxo family of transcription factors regulate the E3 ubiquitin ligases MAFbx and MuRF1. These ligases facilitate the degradation of a many proteins involved in a host of cellular processes like protein synthesis and myoblast differentiation. This suggests that the regulation of these ligases could control the process of muscle atrophy. 


\begin{tabular}{|l|l|l|l|} 
Protein & $\begin{array}{l}\text { Entry into } \\
\text { Torpor }\end{array}$ & $\begin{array}{l}\text { Deep Torpor } \\
\left(4^{\circ} \mathrm{C}\right)\end{array}$ & Arousal \\
\hline NFATc1 & NFATC2 & NFATc3 & Calmodulin
\end{tabular}

Supplementary Table 1: Table summarizing the changes that took place with the western blotting results of the NFAT-calcineurin pathway and their downstream muscle-specific proteins in skeletal muscle. The torpor-arousal cycle including entry into torpor (EN), deep torpor (ET, LT), and arousal (EA, LA, IA) were analyzed in comparison with euthermic control (EC). The green upwards arrow indicates upregulation of protein expression, the red downwards arrow indicates downregulation of protein expression, and the sideways arrow indicates no significant change. 


\section{Protein \\ Entry into \\ Deep Torpor \\ Torpor $\left(4^{\circ} \mathrm{C}\right)$}

\begin{tabular}{|c|c|c|c|}
\hline NFATc1 & 7 & $\Longleftrightarrow$ & 1 \\
\hline NFATc3 & $\Longleftrightarrow$ & $\hat{~}$ & $\Longleftrightarrow$ \\
\hline NFATc4 & 7 & $\Leftrightarrow$ & $\Leftrightarrow$ \\
\hline
\end{tabular}

Supplementary Table 2: Table summarizing the changes that took place with the DPI-ELISA results of NFATc1, c3, and c4 in skeletal muscle. The torpor-arousal cycle including EN, deep torpor (ET, LT), and arousal (EA, LA, IA) were analyzed in comparison with EC. The green upwards arrow indicates upregulation of protein expression, the red downwards arrow indicates downregulation of protein expression, and the sideways arrow indicates no significant change. 


\begin{tabular}{|c|c|c|c|}
\hline Protein & $\begin{array}{l}\text { Entry into } \\
\text { Torpor }\end{array}$ & $\begin{array}{l}\text { Deep Torpor } \\
\left(4^{\circ} \mathrm{C}\right)\end{array}$ & Arousal \\
\hline NFATc1 & $\Longleftrightarrow$ & $\Longleftrightarrow$ & $\Longleftrightarrow$ \\
\hline NFATc2 & $\Longleftrightarrow$ & $\hat{v}$ & $\Longleftrightarrow$ \\
\hline NFATc3 & $\Longleftrightarrow$ & $\Longleftrightarrow$ & $\Longleftrightarrow$ \\
\hline NFATc4 & $\Longleftrightarrow$ & $\Longleftrightarrow$ & $\Longleftrightarrow$ \\
\hline Calmodulin & $\Longleftrightarrow$ & 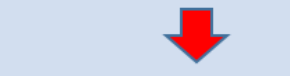 & $v$ \\
\hline Calpain 1 & $\Longleftrightarrow$ & 7 & $\Longleftrightarrow$ \\
\hline Calcineurin & $\Longleftrightarrow$ & $\Longleftrightarrow$ & 7 \\
\hline Myoferlin & $\Longleftrightarrow$ & 1 & $\hat{v}$ \\
\hline Myomaker & $\Longleftrightarrow$ & $\widehat{\vartheta}$ & $\Longleftrightarrow$ \\
\hline
\end{tabular}

Supplementary Table 3: Table summarizing the changes that took place with the western blotting results of the NFAT-calcineurin pathway and their downstream muscle-specific proteins in cardiac muscle. The torpor-arousal cycle including EN, deep torpor (ET, LT), and arousal (EA, LA, IA) were analyzed in comparison with EC. The green upwards arrow indicates upregulation of protein expression, the red downwards arrow indicates downregulation of protein expression, and the sideways arrow indicates no significant change. 


\begin{tabular}{|l|l|l|l|}
\hline Protein & $\begin{array}{l}\text { Entry into } \\
\text { Torpor }\end{array}$ & $\begin{array}{l}\text { Deep Torpor } \\
\left(4^{\circ} \mathrm{C}\right)\end{array}$ & Arousal \\
\hline Ras & &
\end{tabular}

Supplementary Table 4: Table summarizing the changes that took place with the western blotting results of the Ras-Ral pathway, Foxo4 and its phosphorylated forms, as well as Myogenin their downstream E3 ubiquitin ligase proteins in skeletal muscle. The torpor-arousal cycle including EN, deep torpor (ET, LT), and arousal (EA, LA, IA) were analyzed in comparison with EC. The green upwards arrow indicates upregulation of protein expression, the red downwards arrow indicates downregulation of protein expression, and the sideways arrow indicates no significant change. 


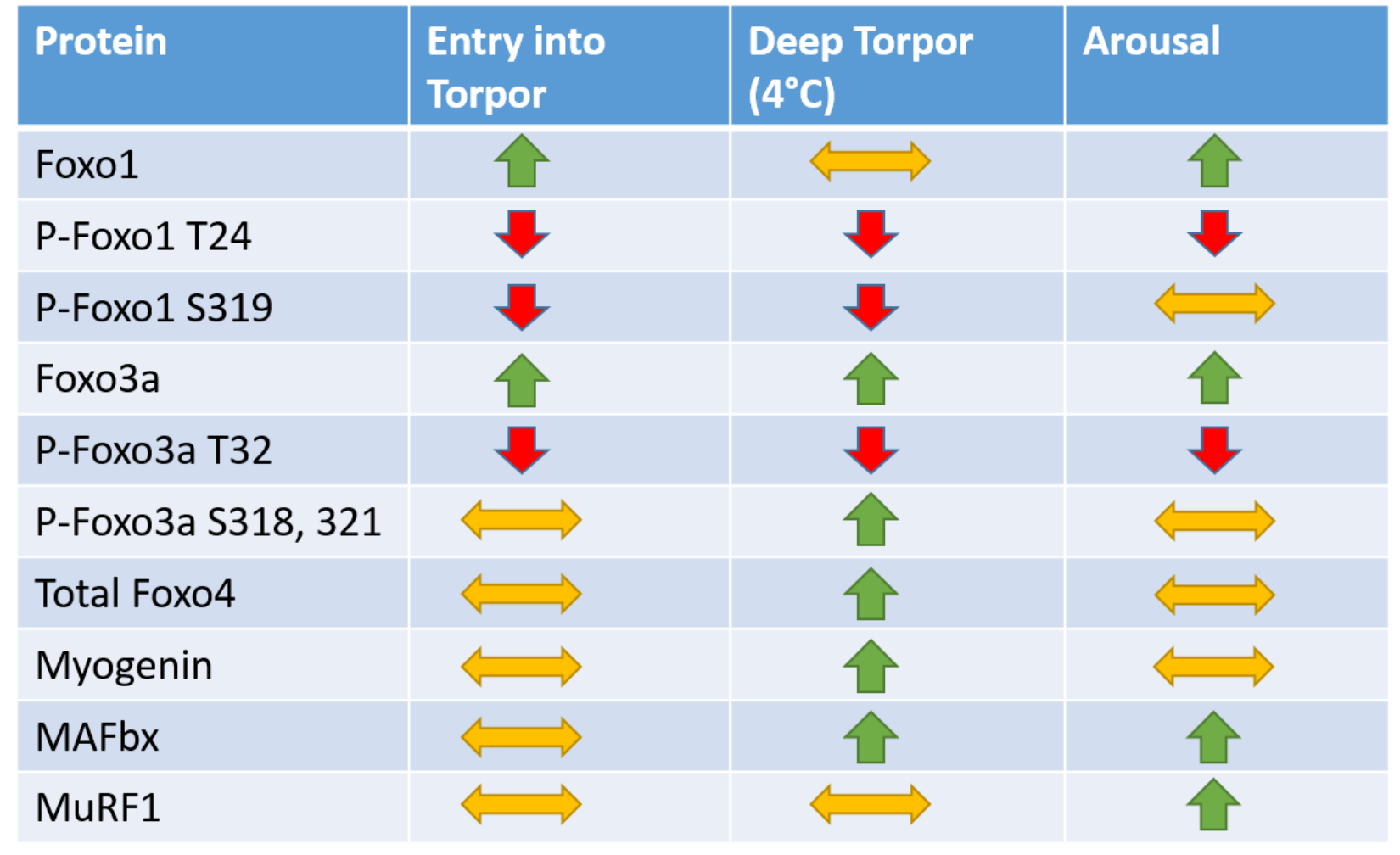

Supplementary Table 5: Table summarizing the changes that took place with the western blotting results of Foxo1, Foxo3a, Foxo4 and its phosphorylated forms, as well as Myogenin their downstream E3 ubiquitin ligase proteins in cardiac muscle. The torpor-arousal cycle including EN, deep torpor (ET, LT), and arousal (EA, LA, IA) were analyzed in comparison with EC. The green upwards arrow indicates upregulation of protein expression, the red downwards arrow indicates downregulation of protein expression, and the sideways arrow indicates no significant change. 


\section{Appendix D: Summary of Contributions}

I have gathered, quantified, analyzed, and interpreted all of the data in this thesis with the exception of selected proteins analyzed via western blotting in Figures 5.3, 6.2, and 6.3. The MAFbx timecourse western blots in Figure 5.3 were done by Dr. Shannon N. Tessier and are unpublished and not part of her thesis. She contributed her data on this target as it was vital to the present chapter and she is a co-author on our submitted manuscript for publication. In Figures 6.2 and 6.3, part of the Foxo3a timecourse data was contributed by Oscar A. Aguilar, although the bands shown were from western blots performed by me. 